\title{
Bost-Connes type systems for function fields
}

\author{
Benoît Jacob
}

\begin{abstract}
We describe a construction which associates to any function field $k$ and any place $\infty$ of $k$ a $C^{*}$-dynamical system $\left(C_{k, \infty}, \sigma_{t}\right)$ that is analogous to the Bost-Connes system associated to $\mathbb{Q}$ and its archimedean place. Our construction relies on Hayes' explicit class field theory in terms of sign-normalized rank one Drinfel'd modules. We show that $C_{k, \infty}$ has a faithful continuous action of $\operatorname{Gal}(K / k)$, where $K$ is a certain field constructed by Hayes such that $k^{\mathrm{ab}, \infty} \subset K \subset k^{\mathrm{ab}}$. Here $k^{\mathrm{ab}, \infty}$ is the maximal abelian extension of $k$ that is totally split at $\infty$. We classify the extremal $\mathrm{KMS}_{\beta}$ states of $\left(C_{k, \infty}, \sigma_{t}\right)$ at any temperature $0<1 / \beta<\infty$ and show that a phase transition with spontaneous symmetry breaking occurs at temperature $1 / \beta=1$. At high temperature $1 / \beta \geqslant 1$, there is a unique $\mathrm{KMS}_{\beta}$ state, of type $\mathrm{III}_{q-\beta}$, where $q$ is the cardinal of the constant subfield of $k$. At low temperature $1 / \beta<1$, the space of extremal $\mathrm{KMS}_{\beta}$ states is principal homogeneous under $\operatorname{Gal}(K / k)$. Each such state is of type $\mathrm{I}_{\infty}$ and the partition function is the Dedekind zeta function $\zeta_{k, \infty}$. Moreover, we construct a *-subalgebra $\mathscr{H}$, we give a presentation of $\mathscr{H}$ and of $C_{k, \infty}$, and we show that the values of the low-temperature extremal $\mathrm{KMS}_{\beta}$ states at certain elements of $\mathscr{H}$ are related to special values of partial zeta functions.
\end{abstract}

Mathematics Subject Classification (2000). 11R58, 58B34.

Keywords. KMS states, explicit class field theory, Drinfel'd modules, $\mathrm{C}^{*}$-dynamical systems.

\section{Contents}

Index of notations . . . . . . . . . . . . . . . . . . . . . . 142

Introduction . . . . . . . . . . . . . . . . . . . . . . . . . 143

1 Function fields, Drinfel'd modules, and Hayes' explicit class field theory . . . . . . 146

2 Construction of the $\mathrm{C}^{*}$-dynamical system $\left(C_{k, \infty},\left(\sigma_{t}\right)\right) \ldots \ldots \ldots \ldots \ldots$

3 Algebraic structure of $\left(C_{k, \infty},\left(\sigma_{t}\right)\right) \ldots \ldots \ldots \ldots \ldots$

$4 \mathrm{KMS}_{\beta}$ equilibrium states of $\left(C_{k, \infty},\left(\sigma_{t}\right)\right) \ldots \ldots \ldots \ldots \ldots \ldots$

References . . . . . . . . . . . . . . . . . . . . . . . . 209 


\section{Index of notations}

\begin{tabular}{|c|c|c|}
\hline $1_{P}(P$ a predicate $), 146$ & $\mathbb{F}_{q}, 147$ & $\begin{array}{l}p, 147 \\
P\left(k, q^{n}\right)\left(n \in \mathbb{N}^{*}\right), 148\end{array}$ \\
\hline$*$ (as in “ $a * \phi ”), 154$ & $g($ genus of $k), 148$ & $\phi_{a}(a \in \mathcal{O}), 153$ \\
\hline$\infty, 143$ & $\mathscr{E}, 163$ & $\phi[a](a \in \mathcal{O}), 154$ \\
\hline$\lfloor x\rfloor(x \in \mathbb{R}), 146$ & $\mathscr{E}_{\chi}(\chi \in X), 163$ & $\begin{array}{l}\phi_{a}\left(a \in \mathfrak{I}_{\mathcal{O}}\right), 154 \\
\phi[a]\left(a \in \mathfrak{I}_{\mathcal{O}}\right), 154\end{array}$ \\
\hline$A_{\mathrm{c}, \sigma}, 191$ & $H, 188$ & $\phi\left(\mathbf{C}_{\infty}\right), 152$ \\
\hline$A_{f}, 147$ & $H^{+}, 157$ & $\phi\left(\mathbf{C}_{\infty}\right)^{\mathrm{tor}}, 154$ \\
\hline$A_{+}, 191$ & $H(\operatorname{sgn}), 157$ & $\phi[F], 193$ \\
\hline$a * \phi\left(a \in \mathfrak{I}_{\mathcal{O}}\right), 154$ & $h(\operatorname{sgn}), 157$ & $\varphi_{\beta}\left(\beta \in \mathbb{R}_{+}^{*}\right), 183$ \\
\hline$\alpha, 196$ & $\mathscr{H}, 165$ & $\tilde{\varphi}_{\beta}\left(\beta \in \mathbb{R}_{+}^{*}\right), 196$ \\
\hline$B(H), 146$ & $\mathscr{H}[\delta], 182$ & $\tilde{\varphi}_{\beta, e}, 203$ \\
\hline$B \ell^{2}, 146$ & $\mathfrak{\Im}_{\mathcal{O}}, 151$ & $\begin{array}{l}\widetilde{\varphi}_{\beta, p, e_{p}}, 203 \\
\varphi_{\beta, \chi}, 188\end{array}$ \\
\hline$C_{1}, 176,194$ & $K, 158$ & $\Phi(\mathfrak{a})\left(a \in \widetilde{\mathfrak{S}}_{\mathcal{O}}\right), 175$ \\
\hline$C_{1, E}, 196$ & $k, 143$ & $\pi_{\chi}(\chi \in X), 164$ \\
\hline$C_{c}(\mathcal{E}), 163$ & $k^{\mathrm{ab}, \infty}, 143$ & \\
\hline $\begin{array}{l}C_{\nu}, 194 \\
C_{m, 151}\end{array}$ & $K_{\beta}\left(\beta \in \mathbb{R}_{+}^{*}\right), 143$ & $O\left(k, q^{n}\right)\left(n \in \mathbb{N}^{*}\right), 148$ \\
\hline$C^{*}\left(\mathfrak{I}_{\mathcal{O}}\right), 175$ & $K_{F}, 193$ & \\
\hline$C_{k, \infty}, 164$ & $\begin{array}{l}K_{\mathrm{c}}\left(\mathrm{c} \in \mathfrak{I}_{\mathcal{O}}\right), 158 \\
k_{\infty}, 147,151\end{array}$ & $\rho_{\mathfrak{p}, \lambda}, 198$ \\
\hline$C_{k, \infty}^{\mathrm{Gat} / K)}, 175$ & & sgn, 157 \\
\hline $\begin{array}{l}C_{\phi}(\phi \in H(\operatorname{sgn})), 171 \\
\chi^{\mathfrak{a}}\left(\chi \in X, a \in \mathfrak{I}_{\mathcal{O}}\right), 159\end{array}$ & $L$ (linear functional), 200 & $\sigma \chi, 160$ \\
\hline$\chi^{c}\left(\chi \in X, c \in \mathfrak{F}_{\mathcal{O}}\right), 161$ & $M, 196$ & $\begin{array}{l}\sigma f, 174 \\
\sigma_{t}(t \in \mathbb{R}), 164\end{array}$ \\
\hline$d(P)$ ( $P$ a set of places), 149 & $\begin{array}{l}M_{1}, 196 \\
M_{1, E}, 196\end{array}$ & $\tilde{\sigma}_{t}(t \in \mathbb{R}), 200$ \\
\hline $\begin{array}{l}\delta(P)(P \text { a set of places }), 149 \\
d \sigma, 176\end{array}$ & $\begin{array}{l}M_{1}^{F \pm}, 199 \\
M_{1, \mathfrak{p}}(\mathfrak{p} \text { a finite place), } 197\end{array}$ & $\begin{array}{l}\tau_{\mathfrak{p}}(\mathfrak{p} \text { a finite place }), 183,197 \\
\theta_{F, v}, 198\end{array}$ \\
\hline$E, 196$ & $M[\mathfrak{D}]\left(\grave{D} \in \mathfrak{I}_{\mathcal{O}}\right), 205$ & \\
\hline $\mathbf{E}, 176$ & $\mathbf{M}(a)\left(a \in \mathfrak{I}_{\mathcal{O}}\right), 175$ & $V, 196$ \\
\hline $\begin{array}{l}\mathcal{E}\left(K_{\beta}\right)\left(\beta \in \mathbb{R}_{+}^{*}\right), 143 \\
e_{L}(L \text { a lattice }), 152 \\
e(\phi, \lambda), 165\end{array}$ & $\begin{array}{l}\mu_{a}\left(a \in \widetilde{I}_{\mathcal{O}}\right), 165 \\
\mu_{\phi}(a)(a \in \mathcal{O}), 154\end{array}$ & $\begin{array}{l}X, 159 \\
X^{\text {adm }}, 178\end{array}$ \\
\hline & $N, 199$ & $X_{F}, 193$ \\
\hline $\mathfrak{F}_{\chi}(\chi \in X), 161$ & $\mathbf{N a}\left(a \in \widetilde{\mathfrak{S}}_{\mathcal{O}}\right), 155$ & $X_{\phi}, 159$ \\
\hline$F_{\searrow}\left(\grave{D} \in \mathfrak{I}_{\mathcal{O}}\right), 182$ & $\mathbf{N c}\left(c \in \mathfrak{F}_{\mathcal{O}}\right), 164$ & \\
\hline $\mathbb{F}_{\infty}, 156$ & $\mathbf{N p}(\mathfrak{p}$ a place of $k), 147$ & $\zeta_{k, \infty}, 149$ \\
\hline $\mathfrak{F} \mathcal{O}, 154$ & & $\zeta_{k, \infty}^{+}, 191$ \\
\hline $\mathbb{F}_{p}, 147$ & $\mathcal{O}, 146$ & $\zeta_{k, \infty}^{\mathrm{c}, \sigma}, 191$ \\
\hline
\end{tabular}




\section{Introduction}

Statement of the main results. Let $k$ be any global function field. Let $\infty$ be any place of $k$. In this paper, we shall associate to the pair $(k, \infty)$ a $C^{*}$-dynamical system $\left(C_{k, \infty},\left(\sigma_{t}\right)\right)$.

Our system aims to be an analog of the Bost-Connes (BC for short) system associated to $\mathbb{Q}$, cf. Bost and Connes [3]. The partition function of the BC system is the Riemann zeta function without the $\Gamma$-factor at infinity. Similarly, we shall check (Lemma 4.3.3) that the partition function of our system is the zeta function of the field $k$ without the factor corresponding to the place $\infty$ of $k$.

The BC system admits $\operatorname{Gal}\left(\mathbb{Q}^{\mathrm{ab}} / \mathbb{Q}\right)$ as symmetry group. Similarly, we shall check (Proposition 3.4.2) that our system has $\operatorname{Gal}(K / k)$ as symmetry group (meaning that $\mathrm{Gal}(K / k)$ acts continuously and faithfully on $C_{k, \infty}$, commuting with the flow $\left.\sigma_{t}\right)$, where $K$ is a field having the following property:

$$
k^{\mathrm{ab}, \infty} \subset K \subset k^{\mathrm{ab}},
$$

where $k^{\mathrm{ab}, \infty}$ is the maximal abelian extension of $k$ that is totally split at $\infty$. The field $K$ is generated over $k$ by coefficients and torsion points of certain rank one Drinfel'd modules; this is part of David R. Hayes' explicit class field theory for function fields, cf. Hayes [18], [19] and [20], which we shall quickly review. If $\infty^{\prime}$ is any place of $k$ other than $\infty$, we have (cf. [18], Theorem 7.2)

$$
k^{\mathrm{ab}, \infty^{\prime}} \cdot k^{\mathrm{ab}, \infty}=k^{\mathrm{ab}} .
$$

We shall construct our $\mathrm{C}^{*}$-algebra $C_{k, \infty}$ as the maximal $\mathrm{C}^{*}$-algebra of a certain groupoid $\mathscr{E}$. We shall also give (Proposition 3.3.6) a presentation of $C_{k, \infty}$ as a $\mathrm{C}^{*}$-algebra.

For any temperature $1 / \beta \in \mathbb{R}_{+}^{*}$, let $K_{\beta}$ be space of $\mathrm{KMS}_{\beta}$ states of $\left(C_{k, \infty},\left(\sigma_{t}\right)\right)$, endowed with the weak* topology. By Bratteli and Robinson [4], II, Theorem 5.3.30, the space $K_{\beta}$ is a compact simplex (in particular, it is convex). Let $\mathcal{E}\left(K_{\beta}\right)$ denote the subspace of extreme points of $K_{\beta}$. The elements of $\mathcal{E}\left(K_{\beta}\right)$ are called the extremal $\mathrm{KMS}_{\beta}$ states. By loc. cit., a $\mathrm{KMS}_{\beta}$ state is extremal if, and only if it is a factor state. Thus, $\mathcal{E}\left(K_{\beta}\right)$ is equal to the space of $\mathrm{KMS}_{\beta}$ factor states.

We shall classify the $\mathrm{KMS}_{\beta}$ states of our system for any temperature $1 / \beta \in \mathbb{R}_{+}^{*}$ : At low temperature $1 / \beta<1$, we shall prove (Theorem 4.3.10) that $\mathscr{E}\left(K_{\beta}\right)$ is principal homogeneous ${ }^{1}$ under $\operatorname{Gal}(K / k)$. The states in $\mathcal{E}\left(K_{\beta}\right)$ are of type $\mathrm{I}_{\infty}$ (Proposition 4.3.8). At high temperature $1 / \beta \geqslant 1$, we shall prove (Theorem 4.4.15) that there exists a unique $\mathrm{KMS}_{\beta}$ state. It is of type $\mathrm{III}_{q^{-\beta}}$ (Theorem 4.5.8), where $q$ is the cardinal of the constant subfield of $k$.

\footnotetext{
${ }^{1}$ Let $G$ be topological group acting on a topological space $X$. One says that $X$ is principal homogeneous under $G$ if, for any $x \in X$, the map $g \mapsto g X$ is a homeomorphism $G \rightarrow X$.
} 
We shall construct a dense $*$-subalgebra $\mathscr{H}$ which gives an arithmetic structure to our dynamical system, as in [3]. For example, we shall show (Theorem 4.3.12) that evaluating low-temperature extremal $\mathrm{KMS}_{\beta}$ states on certain elements of the subalgebra $\mathscr{H}$ gives rise to formulas involving special values of partial zeta functions.

Many of our proofs are adapted from [3], and we have also borrowed several ideas from Harari and Leichtnam [17].

Outline. This paper is divided into four sections. In Section 1 we first review definitions and results in the arithmetic of function fields and in the analytic theory of Drinfel'd modules. We review Hayes' explicit class field theory for function fields, in terms of sign-normalized rank one Drinfel'd modules. We choose once and for all a sign-function sgn, and Hayes' theory provides us with a finite set $H(\operatorname{sgn})$ of Drinfel'd modules with special arithmetic properties. In particular, their coefficients and torsion points generate the extension $K / k$ which we mentioned above. In the rest of this paper, the only Drinfel'd modules which we consider are the elements of $H$ (sgn).

In Section 2 we do the actual construction of the $C^{*}$-dynamical system $\left(C_{k, \infty},\left(\sigma_{t}\right)\right)$. From the finite set $H(\mathrm{sgn})$ provided by Hayes' theory, we construct a compact topological space $X$ in the following way: for any $\phi \in H(\operatorname{sgn})$, let $X_{\phi}$ denote the dual group of the discrete group of torsion points of the Drinfel'd module $\phi$. Let $X$ be the disjoint union of the $X_{\phi}$, where $\phi$ runs over $H(\operatorname{sgn})$. The compact space $X$ is endowed with a natural action of the semigroup $\mathfrak{\Im}_{\mathcal{O}}$ of ideals. This gives rise to a groupoid $\mathscr{E}$, and the $\mathrm{C}^{*}$-algebra $C_{k, \infty}$ is obtained as the maximal groupoid $\mathrm{C}^{*}$-algebra of $\mathcal{E}$. The flow $\left(\sigma_{t}\right)$ is then easy to define.

In Section 3 we prove a number of results about the algebraic structure of $\left(C_{k, \infty},\left(\sigma_{t}\right)\right)$. We introduce a $*$-subalgebra $\mathscr{H}$ which plays the rôle of the algebra $\mathscr{H}$ in the paper [3]. We prove that $\mathscr{H}$ is dense in $C_{k, \infty}$, and we give a presentation of $\mathcal{H}$ as a $*$-algebra and of $C_{k, \infty}$ as a $\mathrm{C}^{*}$-algebra. We then study an action of $\operatorname{Gal}(K / k)$ on $C_{k, \infty}$ and compute the fixed-point subalgebra $C_{1}$. The rest of this section is devoted to miscellaneous arithmetical results which we use in the last section.

In Section 4 for any temperature $1 / \beta \in \mathbb{R}_{+}^{*}$, we describe the space $\mathcal{E}\left(K_{\beta}\right)$ of extremal $\mathrm{KMS}_{\beta}$ states (endowed with the weak ${ }^{*}$ topology), and we compute the type of all such states. We first construct a $\mathrm{KMS}_{\beta}$ state $\varphi_{\beta}$ and show that it is the unique $\operatorname{Gal}(K / k)$-invariant $\mathrm{KMS}_{\beta}$ state. We then show that the action of $\mathrm{Gal}(K / k)$ on $\mathcal{E}\left(K_{\beta}\right)$ is transitive and continuous. Thus, in order to describe $\mathcal{E}\left(K_{\beta}\right)$, it is enough to find an element of $\mathscr{E}\left(K_{\beta}\right)$ and to describe its orbit under $\operatorname{Gal}(K / k)$. At low temperature $1 / \beta<1$, we associate to any admissible character $\chi$ a Gibbs state $\varphi_{\beta, \chi}$ in the regular representation at $\chi$. We prove that the map $\chi \mapsto \varphi_{\beta, \chi}$ is a homeomorphism from the space $X^{\text {adm }}$ of admissible characters to $\mathcal{E}\left(K_{\beta}\right)$. We also prove that both spaces are principal homogeneous under $\operatorname{Gal}(K / k)$. We check that the states in $\mathcal{E}\left(K_{\beta}\right)$ are of type $\mathrm{I}_{\infty}$, that the partition function is the Dedekind zeta 
function $\zeta_{k, \infty}$, and we compute the values of the $\varphi_{\beta, \chi}$ at some points of $\mathscr{H}$ in terms of special values at $\beta$ of partial zeta functions of $k$. At high temperature $1 / \beta \geqslant 1$, we prove that $\mathscr{E}\left(K_{\beta}\right)=\left\{\varphi_{\beta}\right\}$ and that the type of $\varphi_{\beta}$ is III $_{q-\beta}$, where $q$ is the cardinal of the constant subfield of $k$.

Literature on Bost-Connes type constructions. The 1995 paper [3] has inspired many mathematicians. Unfortunately, it would be impossible to mention all of them here; we refer to Section 1.4 of Connes and Marcolli [11] for a more complete summary. M. Laca, N. Larsen, I. Raeburn and others have investigated in a number of papers (see for instance [2], [25], [26], [27], [28]) the semigroup crossed product and Hecke algebra aspects of the BC construction and generalizations of it. In 1997, D. Harari and E. Leichtnam have obtained in [17] a system with spontaneous symmetry breaking for any global field. In 1999, P. Cohen has obtained in [6] a system for number fields whose partition function is the Dedekind zeta function. In 2002, $\mathrm{S}$. Neshveyev has given in [29] a new proof of the uniqueness of the $\mathrm{KMS}_{\beta}$ state at high temperature. In 2004, A. Connes and M. Marcolli have introduced in [11] the noncommutative space of $\mathbb{Q}$-lattices up to scaling and commensurability, allowing for a comprehensive reformulation of the $\mathrm{BC}$ construction, and have studied the case of rank 2. In 2005, A. Connes, M. Marcolli and N. Ramachandran have obtained in [12], [13] the "good" system for quadratic imaginary number fields and have studied its relation to complex multiplication of elliptic curves. The same year, E. Ha and F. Paugam have extended in [16] the Connes-Marcolli setting to arbitrary Shimura varieties. Finally, in the paper [10], A. Connes, C. Consani and M. Marcolli have introduced the notion of an endomotive, putting the BC construction into a much wider perspective which also includes A. Connes' spectral realization [9] of the zeroes of the Riemann zeta function.

Acknowledgements. I thank Éric Leichtnam for giving me this research subject, for many helpful comments on early versions of this paper, and for his explanations on operator algebras. I thank Matilde Marcolli for encouraging me to give a talk on this material at MPI, Bonn, in October 2005. During this research, I enjoyed the excellent working environment of the "Projet Algèbres d'Opérateurs" at Jussieu, and I thank Étienne Blanchard for letting me talk in its seminar. I am very grateful to Alain Connes, David R. Hayes, Georges Skandalis and Stefaan Vaes who kindly answered mathematical questions. I also thank Cécile Armana, Pierre Fima, Eugene Ha, Cyril Houdayer and Frédéric Paugam for helpful discussions.

Here are two interesting remarks that people made at the end of my MPI talk.

1. As Alain Connes pointed out, our system lacks one feature of the BC system: fabulous states. The reason for that is obvious: values of states are elements of $\mathbb{C}$, so the symmetry group $\operatorname{Gal}(K / k)$ does not act naturally on them. Obtaining fabulous states would require to have a theory of dynamical systems of positive characteristic, where states would take values in some field of positive characteristic. Note that even 
though the low temperature extremal $\mathrm{KMS}_{\beta}$ states of our system do not have the fabulous property, they have interesting special values (Theorem 4.3.12).

2. Arkady Kholodenko mentioned that it might be possible to adapt his work on $2+1$ gravity [23] in order to obtain zeta functions of function fields as partition functions, and that Drinfel'd modules should play a rôle.

Notations. In this paper, $\mathbb{N}$ denotes the set of nonnegative integers, $\mathbb{N}^{*}$ denotes the set of positive integers, and $\mathbb{R}_{+}^{*}$ denotes the set of positive real numbers. Thus $0 \in \mathbb{N}$, $0 \notin \mathbb{N}^{*}$, and $0 \notin \mathbb{R}_{+}^{*}$. For any Hilbert space $H$, we let $B(H)$ denote the algebra of all bounded linear operators on $H$. For any set $X$, we write $B \ell^{2}(X)$ for $B\left(\ell^{2}(X)\right)$. For any $x \in \mathbb{R}$, we set

$$
\lfloor x\rfloor=\max \{n \in \mathbb{Z} \mid n \leqslant x\} .
$$

For any predicate $P$, we define $1_{P}$ to be equal to 1 if $P$ is true, and 0 if $P$ is false. Thus, we have for any two predicates $P$ and $Q$ :

$$
1_{P} \text { and } Q=1_{P} 1_{Q} .
$$

\section{Function fields, Drinfel'd modules, and Hayes' explicit class field theory}

1.1. Function fields. Here are three equivalent definitions of a function field:

- A field which is a finite extension of $\mathbb{F}_{p}(T)$, for some prime number $p$.

- A global field of positive characteristic.

- The field $K(C)$ of rational functions on a projective curve $C$ over a finite field. The curve $C$ can always be chosen to be smooth.

Thus, global fields fall into two categories: those of characteristic 0 are the number fields, and those of positive characteristic are the function fields.

Recall that at the beginning of this paper, we chose a function field $k$ and a place $\infty$ of $k$.

Function fields have many similarities with number fields. An important part of algebraic number theory works in the same way for all global fields.

The analog of the Dedekind ring of integers is defined as follows. According to the third definition of a function field, view $k$ as the field $K(C)$ of rational functions on a smooth projective curve $C$ over a finite field. View $\infty$ as a closed point of $C$. Let $\mathcal{O}$ be the subring of $k$ of all functions having no pole away from $\infty$. In other words, $\mathcal{O}$ is the ring of regular functions on the affine curve $C-\{\infty\}$. Note that $k=K(C)$ is the field of fractions of $\mathcal{O}$.

Example. $k=\mathbb{F}_{p}(T)$ and $\infty$ is the place corresponding to an absolute value $|\cdot|$ such that $|T|>1$. The subring $\mathcal{O}$ is then the polynomial ring $\mathbb{F}_{p}[T]$. 
Call finite the places of $k$ other than $\infty$. We have a natural bijection

finite places of $k \longleftrightarrow$ maximal ideals of $\mathcal{O}$.

Let $p$ denote the characteristic of $k$. The range of the unique unital ring morphism $\mathbb{Z} \rightarrow k$ is a finite field with $p$ elements; we denote it by $\mathbb{F}_{p}$. The algebraic closure of $\mathbb{F}_{p}$ in $k$ is called the constant subfield of $k$. Let $q$ denote its cardinal. Of course, $q$ is a power of $p$. We let $\mathbb{F}_{q}$ denote the constant subfield of $k$. An element of $k$ is said to be constant if it belongs to $\mathbb{F}_{q}$.

For any place $\mathfrak{p}$ of $k$, we let $\mathbf{N p}$ denote the cardinal of the residue field of $\mathfrak{p}$. Thus, $\mathbf{N p}=q^{n_{\mathfrak{p}}}$ for some positive integer $n_{\mathfrak{p}}$ called the degree of $\mathfrak{p}$. Note that if $\mathfrak{p}$ is finite, then the residue field is the quotient $\mathcal{O} / \mathfrak{p}$.

The rest of this subsection is a review of a few well-known theorems about function fields, which will be used in the proofs of our classification of $\mathrm{KMS}_{\beta}$ states. These theorems are: the strong approximation theorem, Weil's "Riemann Hypothesis for curves", and the abelian case of the Čebotarev density theorem for the natural density. The first one will be used in Subsection 3.6, which in turn will be used in the classification of $\mathrm{KMS}_{\beta}$ states at low temperature. The two other ones will be used in the classification of $\mathrm{KMS}_{\beta}$ states at high temperature.

Let $A_{f}$ denote the ring of finite adèles of $k$. This is the restricted product of the $k_{\mathfrak{p}}$ with respect to the $\mathcal{O}_{\mathfrak{p}}$, where $\mathfrak{p}$ runs over all finite places of $k$. Let $\iota_{f}: k \hookrightarrow A_{f}$ be the diagonal embedding.

Theorem 1.1.1 (Strong approximation theorem). The field $\iota_{f}(k)$ is dense in $A_{f}$.

Proof. See Cassels and Fröhlich [5], Chapter II, §15, p. 67.

This is contrasted with the fact that if $\iota: k \hookrightarrow A$ is the diagonal embedding into the full ring of adèles, then $\iota(k)$ is discrete in $A$. Note that

$$
A=A_{f} \times k_{\infty}
$$

where $k_{\infty}$ is the completion of $k$ at $\infty$.

Let us now recall Weil's "Riemann hypothesis for curves" theorem. The genus of a function field is the genus of any projective smooth curve of which it is the function field. For the statement of the following theorem, we temporarily forget that we already chose a function field $k$ and defined $q$ as the cardinal of its constant subfield.

Theorem 1.1.2 (A. Weil, the Riemann Hypothesis for curves). Let $k$ be a function field of genus $g$. Let $q$ be the cardinal of its constant subfield. Let $N$ be the number of places of $k$ with norm $q$ (i.e. with degree 1). Then

$$
q-2 g \sqrt{q}+1 \leqslant N \leqslant q+2 g \sqrt{q}+1 .
$$


Proof. Weil's original proof is published in [34].

Let us now come back to the function field $k$ that we fixed at the beginning of this paper. Let $g$ denote the genus of $k$.

Given an integer $n \geqslant 1$, one may ask how to obtain a result similar to Theorem 1.1.2 for places of $k$ with norm $q^{n}$ (i.e. with degree $n$ ). Note that one cannot replace $q$ by $q^{n}$ in Theorem 1.1.2. Here one has to be wary of the distinction between closed points, which correspond to places of $k$, and geometric points, which correspond to places of suitable extensions of $k$. The following corollary will be used in Subsections 4.4 and 4.5.

Corollary 1.1.3. For any $n \geqslant 1$, let $Q\left(k, q^{n}\right)$ denote the number of places of $k$ with norm $q^{n}$, and let $P\left(k, q^{n}\right)$ denote the number of places $k$ with norm $\leqslant q^{n}$. The following estimates hold when $n \rightarrow \infty$ :

$$
\begin{aligned}
& Q\left(k, q^{n}\right)=\frac{q^{n}}{n}+O\left(q^{n / 2}\right), \\
& P\left(k, q^{n}\right) \sim \frac{q}{q-1} \cdot \frac{q^{n}}{n} .
\end{aligned}
$$

Proof. For any $n \geqslant 1$, let $k_{n}=k \otimes \mathbb{F}_{q} \mathbb{F}_{q^{n}}$. Note that the constant subfield of $k_{n}$ is $\mathbb{F}_{q^{n}}$. Let $N_{n}$ denote the number of places of $k_{n}$ with norm $q^{n}$. By Theorem 1.1.2 applied to the function field $k_{n}$, we have

$$
q^{n}-2 g q^{n / 2}+1 \leqslant N_{n} \leqslant q^{n}+2 g q^{n / 2}+1 .
$$

Let $n \geqslant 1$. One easily checks that for any $m \mid n$ there is a bijection

$$
\begin{aligned}
\text { places of } k \text { with norm } q^{m} \longleftrightarrow \operatorname{Gal}\left(k_{n} / k\right) \text {-orbits with cardinal } m \\
\text { of places of } k_{n} \text { with norm } q^{n} .
\end{aligned}
$$

Thus we have

$$
N_{n}=\sum_{m \mid n} m Q\left(k, q^{m}\right)
$$

This gives

$$
n Q\left(k, q^{n}\right)=N_{n}-\sum_{m \mid n, m \leqslant n / 2} m Q\left(k, q^{m}\right) .
$$

By equation (4), we have $m Q\left(k, q^{m}\right) \leqslant N_{m}$, so we find

$$
\begin{aligned}
N_{n} \geqslant n Q\left(k, q^{n}\right) & \geqslant N_{n}-\sum_{m \mid n, m \leqslant n / 2} N_{m} . \\
& \geqslant N_{n}-(n / 2) N_{\lfloor n / 2\rfloor} .
\end{aligned}
$$


Applying the inequality (3), we get

$$
q^{n}+2 g q^{n / 2}+1 \geqslant n Q\left(k, q^{n}\right) \geqslant q^{n}-2 g q^{n / 2}+1-(n / 2)\left(q^{n / 2}+2 g q^{n / 4}+1\right),
$$

and the estimate (1) follows. From the estimate (1), using the equality

$$
P\left(k, q^{n}\right)=\sum_{m=1}^{n} Q\left(k, q^{m}\right)
$$

one can obtain the estimate (2) by an elementary computation.

For any $s \in \mathbb{C}$ with $\operatorname{Re} s>1$, put

$$
\zeta_{k}(s)=\prod_{\mathfrak{p}} \frac{1}{1-\mathbf{N p}^{-s}}
$$

where the product is taken over all places of $k$. One shows that $\zeta_{k}$ can be continued to a meromorphic function on $\mathbb{C}$. Note that $\zeta_{k}$ is periodic, with period $2 \pi i / \log q$. The inequality (3) for all $n \geqslant 1$ is then equivalent to the statement that all zeroes of $\zeta_{k}$ have real part $1 / 2$. One defines the zeta function without the factor at $\infty$, denoted by $\zeta_{k, \infty}$, to be the meromorphic continuation of the function defined when $\operatorname{Re} s>1$ by

$$
\zeta_{k, \infty}(s)=\prod_{\mathfrak{p} \neq \infty} \frac{1}{1-\mathbf{N p}^{-s}}=\left(1-\mathbf{N} \infty^{-s}\right) \zeta_{k}(s) .
$$

Note that when $\operatorname{Re} s>1$, we have

$$
\zeta_{k, \infty}(s)=\sum_{a \in \mathfrak{I}_{\mathcal{O}}} \frac{1}{\mathbf{N a}^{s}}
$$

Let us now recall a version of the Čebotarev density theorem.

Let $S$ denote the set of all places of $k$. A set $P$ of places of $k$ is said to have $a$ Dirichlet density if the following limit exists in $\mathbb{R}$ :

$$
d(P)=\lim _{s \rightarrow 1_{+}} \frac{\sum_{\mathfrak{p} \in P} \mathbf{N p}^{-s}}{\sum_{\mathfrak{p} \in S} \mathbf{N p}^{-s}} .
$$

Moreover, $P$ is said to have a natural density if the following limit exists in $\mathbb{R}$ :

$$
\delta(P)=\lim _{N \rightarrow+\infty} \frac{\operatorname{Card}\{\mathfrak{p} \in P \mid \mathbf{N} \mathfrak{p} \leqslant N\}}{\operatorname{Card}\{\mathfrak{p} \in S \mid \mathbf{N} \mathfrak{p} \leqslant N\}} .
$$

If a set $P$ has a natural density, then it also has a Dirichlet density, and $d(P)=\delta(P)$. 
Theorem 1.1.4 (Čebotarev density theorem, abelian case, for the natural density). Let $L$ be a finite abelian extension of $k$. Let $\sigma \in \operatorname{Gal}(L / k)$. Let $P$ denote the set of all places $\mathfrak{p}$ of $k$ unramified in $L$ and such that $\sigma_{\mathfrak{p}}=\sigma$, where $\sigma_{\mathfrak{p}}=(\mathfrak{p}, L / k) \in$ $\operatorname{Gal}(L / k)$ is the Artin automorphism of $L$ associated to $p$. Then $P$ has natural density $\delta(P)=1 /[L: k]$. Therefore, it also has Dirichlet density $d(P)=1 /[L: k]$.

Proof. Combine [5], Chapter VIII, Theorem 4 with the Artin reciprocity law.

We shall use the Čebotarev density theorem in Subsection 4.4, and we shall also use the following corollary in Subsection 4.5.

Corollary 1.1.5. Let $L$ be a finite abelian extension of $k$. Let $\sigma \in \operatorname{Gal}(L / k)$. For any $n \geqslant 1$, let $P\left(L / k, q^{n}, \sigma\right)$ denote the number of places of $\mathfrak{p}$ of $k$ unramified in $L$ such that $\mathbf{N} \mathfrak{p} \leqslant q^{n}$ and $\sigma_{\mathfrak{p}}=\sigma$, where $\sigma_{\mathfrak{p}}=(\mathfrak{p}, L / k) \in \operatorname{Gal}(L / k)$ is the Artin automorphism of $L$ associated to $\mathfrak{p}$. Let $Q\left(L / k, q^{n}, \sigma\right)$ denote the number of places of $\mathfrak{p}$ of $k$ unramified in $L$ such that $\mathbf{N p}=q^{n}$ and $\sigma_{\mathfrak{p}}=\sigma$. The following estimates hold when $n \rightarrow \infty$ :

$$
\begin{aligned}
& P\left(L / k, q^{n}, \sigma\right) \sim \frac{q}{(q-1)[L: k]} \cdot \frac{q^{n}}{n}, \\
& Q\left(L / k, q^{n}, \sigma\right) \sim \frac{1}{[L: k]} \cdot \frac{q^{n}}{n} .
\end{aligned}
$$

Proof. The estimate (5) follows from Theorem 1.1.4 and the estimate (2). We have

$$
Q\left(L / k, q^{n}, \sigma\right)=P\left(L / k, q^{n}, \sigma\right)-P\left(L / k, q^{n-1}, \sigma\right),
$$

so

$$
\frac{Q\left(L / k, q^{n}, \sigma\right)}{P\left(k, q^{n}\right)}=\frac{P\left(L / k, q^{n}, \sigma\right)}{P\left(k, q^{n}\right)}-\frac{P\left(L / k, q^{n-1}, \sigma\right)}{P\left(k, q^{n-1}\right)} \cdot \frac{P\left(k, q^{n-1}\right)}{P\left(k, q^{n}\right)} .
$$

Hence

$$
\frac{Q\left(L / k, q^{n}, \sigma\right)}{P\left(k, q^{n}\right)} \stackrel{n \rightarrow \infty}{\longrightarrow} \frac{1}{[L: k]}-\frac{1}{[L: k]} \cdot \frac{1}{q}=\frac{q-1}{q[L: k]} .
$$

Applying the estimate (2) to that, we get the estimate (6).

1.2. Drinfel'd modules over $\mathbf{C}_{\infty}$. Our references in this subsection are [20] and Chapter IV of Goss [15].

Recall that the maximal abelian extension of a quadratic imaginary number field is generated by the $j$-invariant and the torsion points of a suitable elliptic curve over $\mathbb{C}$. One wishes to develop a similar theory for function fields. Thus, one looks for good analogs of $\mathbb{C}$ and of the notion of an elliptic curve over $\mathbb{C}$. The analog of the field $\mathbb{C}$ has been well known for a long time and is what we shall denote $\mathbf{C}_{\infty}$. The analog of 
the notion of an elliptic curve over $\mathbb{C}$ is going to be the notion of a Drinfel'd module over $\mathbf{C}_{\infty}$.

We begin with describing the analog of $\mathbb{C}$. Let $k_{\infty}$ be the completion of $k$ at $\infty$. The problem is that $k_{\infty}$ is not algebraically closed. Take an algebraic closure $k_{\infty}^{\text {alg }} / k_{\infty}$. One shows that $\infty$ extends uniquely to a place of $k_{\infty}^{\text {alg }}$. Then the problem is that $k_{\infty}^{\text {alg }}$ is not complete. So let $\mathbf{C}_{\infty}$ denote the completion of $k_{\infty}^{\mathrm{alg}}$ at $\infty$. The field $\mathbf{C}_{\infty}$ is both complete and algebraically closed.

Let us choose once and for all an imbedding $\iota: k \hookrightarrow \mathbf{C}_{\infty}$, and use it to view $k$ as a subfield of $\mathbf{C}_{\infty}$.

Lattices. We are now ready to introduce Drinfel'd modules. The most concrete way to introduce elliptic curves over $\mathbb{C}$ is to first define lattices in $\mathbb{C}$. Similarly, we are going to first define lattices in $\mathbf{C}_{\infty}$.

Recall that $\mathcal{O}$ is the subring of integers of $k$, defined in the previous subsection. A subgroup $L \subset \mathbf{C}_{\infty}$ is said to be discrete if there exists a neighborhood $U$ of 0 in $\mathbf{C}_{\infty}$ such that $U \cap L=\{0\}$.

Definition 1.2.1. An $\mathcal{O}$-lattice in $\mathbf{C}_{\infty}$ is a discrete, finitely generated $\mathcal{O}$-submodule of $\mathbf{C}_{\infty}$.

We shall say "lattice" instead of " $\mathcal{O}$-lattice in $\mathbf{C}_{\infty}$ ".

This is an abstract definition, but in this paper we shall only have to deal with a special case of lattices, rank one lattices, for which there is a very concrete definition. Let us first define the rank of a lattice.

Let $L$ be a lattice. As $\mathbf{C}_{\infty}$ is a field containing $\mathcal{O}$, it is obviously a torsion-free $\mathcal{O}$-module. Hence $L$ is also torsion-free. As $\mathcal{O}$ is a Dedekind ring, the $\mathcal{O}$-module $L$, being finitely generated and torsion-free, is automatically projective, so there exist an integer $r \geqslant 1$ and ideals $a_{1}, \ldots, a_{r} \in \mathfrak{I}_{\mathcal{O}}$ such that $L$ is isomorphic as an $\mathcal{O}$-module to $a_{1} \oplus \cdots \oplus a_{r}$.

Definition 1.2.2. The integer $r$ above is called the rank of $L$.

Let $\mathfrak{S}_{\mathcal{O}}$ be the semigroup of all nonzero ideals of $\mathcal{O}$, under the usual multiplication law of ideals. For rank one lattices, we have the following result:

A subset of $\mathbf{C}_{\infty}$ is a rank one lattice if, and only if it is of the form $\xi$ a with $\xi \in \mathbf{C}_{\infty}^{*}$ and $a \in \mathfrak{s}_{\mathcal{O}}$.

The Drinfel'd module associated to a lattice. Let $L$ be a lattice (of any rank). Remember the following product formula:

$$
\sin z=z \prod_{t \in \pi \mathbb{Z}-\{0\}}(1-z / t) \quad \text { for all } z \in \mathbb{C} .
$$


Similarly, let us define a function $e_{L}: \mathbf{C}_{\infty} \rightarrow \mathbf{C}_{\infty}$ by the following formula:

$$
e_{L}(x)=x \prod_{\ell \in L-\{0\}}(1-x / \ell) \text { for all } x \in \mathbf{C}_{\infty} \text {. }
$$

One shows that this product converges for all $x$. The function $e_{L}$ should be called the "sinus function associated to $L$ ", but authors have decided to call it the "exponential function associated to $L "$. We have

$$
e_{L}(x+y)=e_{L}(x)+e_{L}(y) \text { for all } x, y \in \mathbf{C}_{\infty},
$$

and

$$
e_{L}(a x)=\phi_{a}^{L}\left(e_{L}(x)\right) \text { for all } a \in \mathcal{O}, x \in \mathbf{C}_{\infty},
$$

where $\phi_{a}^{L} \in \mathbf{C}_{\infty}[X]$ is the polynomial given by the following formula if $a \neq 0$ :

$$
\phi_{a}^{L}=a X \prod_{0 \neq \ell \in a^{-1} L / L}\left(1-X / e_{L}(\ell)\right),
$$

and $\phi_{0}^{L}=0$. Note that if $a$ is a nonzero constant (that is, $a \in \mathbb{F}_{q}^{*}$ ), then it is invertible in $\mathcal{O}$ and hence $a^{-1} L=L$. Thus, one has

$$
\phi_{a}=a X \quad \text { for all } a \in \mathbb{F}_{q} .
$$

As we shall shortly see, this allows to check that for any $a \in \mathcal{O}$ the polynomial $\phi_{a}^{L}$ is $\mathbb{F}_{q}$-linear, which means that it can have nonzero coefficients only in degrees that are powers of $q$.

Equation (7) is an analog of the classical formula for $\sin (x+y)$, not of the formula for $\exp (x+y)$. The fact that $e_{L}$ is additive, while sin is not, is a phenomenon typical of characteristic $p$ algebra, just like the additivity of the Frobenius map $x \mapsto x^{p}$. The polynomials $\phi_{a}^{L}$ can be viewed as analogs of the classical Chebycheff polynomials of trigonometry.

One shows, by analytic means, that $e_{L}$ induces a bijection

$$
e_{L}: \mathbf{C}_{\infty} / L \rightarrow \mathbf{C}_{\infty}
$$

So this is a group isomorphism. Use it to transport the $\mathcal{O}$-module structure of $\mathbf{C}_{\infty} / L$ to a new $\mathcal{O}$-module structure on $\mathbf{C}_{\infty}$, which we denote $\phi^{L}\left(\mathbf{C}_{\infty}\right)$. Thus, $\phi^{L}\left(\mathbf{C}_{\infty}\right)$ is the $\mathcal{O}$-module that is equal to $\mathbf{C}_{\infty}$ as an additive group and whose $\mathcal{O}$-module structure is given by

$$
(a, x) \mapsto \phi_{a}^{L}(x) .
$$

Thus, by definition, the map $e_{L}$ is an isomorphism of $\mathcal{O}$-modules

$$
e_{L}: \mathbf{C}_{\infty} / L \rightarrow \phi^{L}\left(\mathbf{C}_{\infty}\right)
$$

The Drinfel'd module associated to $L$ is the map

$$
\begin{aligned}
\phi^{L}: \mathcal{O} & \rightarrow \mathbf{C}_{\infty}[X], \\
a & \mapsto \phi_{a}^{L} .
\end{aligned}
$$


Definition of a Drinfel'd module over $\mathbf{C}_{\infty}$. The map $\phi^{L}$ that we have just defined satisfies

$$
\begin{aligned}
\phi_{a+b}^{L} & =\phi_{a}^{L}+\phi_{b}^{L} \quad \text { for all } a, b \in \mathcal{O}, \\
\phi_{a b}^{L} & =\phi_{a}^{L} \circ \phi_{b}^{L}=\phi_{b}^{L} \circ \phi_{a}^{L} \quad \text { for all } a, b \in \mathcal{O} .
\end{aligned}
$$

Let $\tau=X^{q}$ and, for $n \geqslant 0, \tau^{n}=X^{q^{n}}$. In particular, $\tau^{0}=X$. Let $\mathbf{C}_{\infty}\{\tau\}$ denote the (noncommutative) $\mathbf{C}_{\infty}$-algebra whose underlying vector space is the $\mathbf{C}_{\infty}$-linear span of the $\tau^{n}$, for $n \geqslant 0$, and where the "multiplication" law is the composition law $\circ$. Note that $\mathbf{C}_{\infty}\{\tau\}$ consists exactly of those polynomials that are $\mathbb{F}_{q}$-linear. Combining equations (9) and (12), one obtains that the polynomial $\phi_{a}^{L}$ is $\mathbb{F}_{q}$-linear,

$$
\phi_{a}^{L} \in \mathbf{C}_{\infty}\{\tau\} \quad \text { for all } a \in \mathcal{O},
$$

and that the map $\mathcal{O} \rightarrow \mathbf{C}_{\infty}\{\tau\}, a \mapsto \phi_{a}^{L}$, is $\mathbb{F}_{q}$-linear as well. Thus, it is a morphism of $\mathbb{F}_{q}$-algebras

$$
\begin{aligned}
\phi^{L}: \mathcal{O} & \rightarrow \mathbf{C}_{\infty}\{\tau\}, \\
a & \mapsto \phi_{a}^{L} .
\end{aligned}
$$

Let

$$
D: \mathbf{C}_{\infty}\{\tau\} \rightarrow \mathbf{C}_{\infty}
$$

be the derivative-at-0 map. In other words, $D$ is the $\mathbf{C}_{\infty}$-linear map defined by $D\left(\tau^{0}\right)=1$ and $D\left(\tau^{n}\right)=0$ for any $n \geqslant 1$. We have

$$
D\left(\phi_{a}^{L}\right)=a \quad \text { for all } a \in \mathcal{O} .
$$

This leads to the general definition of a Drinfel'd module over $\mathbf{C}_{\infty}$ :

Definition 1.2.3. Let $\phi: \mathcal{O} \rightarrow \mathbf{C}_{\infty}\{\tau\}, a \mapsto \phi_{a}$, be a morphism of $\mathbb{F}_{q}$-algebras. Then $\phi$ is a Drinfel'd module over $\mathbf{C}_{\infty}$ if and only if

(1) for all $a \in \mathcal{O}, D\left(\phi_{a}\right)=a$,

(2) $\phi$ is non-trivial, i.e. $\phi$ is not the map $a \mapsto a \tau^{0}$.

To any lattice $L$ of any rank we have associated a Drinfel'd module over $\mathbf{C}_{\infty}$, which we denoted by $\phi^{L}$. The uniformization theorem states that any Drinfel'd module over $\mathbf{C}_{\infty}$ comes from a unique lattice. Thus, the map $L \mapsto \phi^{L}$ is a bijection between lattices and Drinfel'd modules over $\mathbf{C}_{\infty}$.

The rank of a Drinfel'd module over $\mathbf{C}_{\infty}$ is the rank of the associated lattice. 
Action of the ideals. For any Drinfel'd module $\phi$ over $\mathbf{C}_{\infty}$ and any $a \in \mathfrak{I}_{\mathcal{O}}$, we define the polynomial $\phi_{\mathfrak{a}} \in \mathbf{C}_{\infty}\{\tau\}$ as follows. Let $I_{\mathfrak{a}, \phi}$ be the left ideal of $\mathbf{C}_{\infty}\{\tau\}$ generated by the $\phi_{a}$, for $a \in a$. One can show that every left ideal of $\mathbf{C}_{\infty}\{\tau\}$ is principal, so there exists a unique monic $\phi_{\mathfrak{a}} \in \mathbf{C}_{\infty}\{\tau\}$ such that $I_{\mathfrak{a}, \phi}=\mathbf{C}_{\infty}\{\tau\} \phi_{\mathfrak{a}}$.

For any Drinfel'd module $\phi$ over $\mathbf{C}_{\infty}$ and any $a \in \mathcal{O}$, we define an element $\mu_{\phi}(a) \in \mathbf{C}_{\infty}^{*}$ by

$$
\mu_{\phi}(a)=\text { leading (highest-degree) coefficient of the polynomial } \phi_{a} .
$$

Note that if $a$ is a principal ideal of $\mathcal{O}$, for any $a \in \mathcal{O}$ such that $a=a \mathcal{O}$, we have

$$
\phi_{\mathfrak{a}}=\mu_{\phi}(a)^{-1} \phi_{a} .
$$

It is easy to see that for any $b \in \mathcal{O}$, we have $I_{\mathfrak{a}, \phi} \phi_{b} \subset I_{\mathfrak{a}, \phi}$. Thus, for any $b \in \mathcal{O}$ we have $\phi_{a} \phi_{b} \in I_{a, \phi}$, so there is a unique $\phi_{b}^{\prime} \in \mathbf{C}_{\infty}\{\tau\}$ such that

$$
\phi_{a} \phi_{b}=\phi_{b}^{\prime} \phi_{a} \text {. }
$$

One shows that the map $b \mapsto \phi_{b}^{\prime}$ is a Drinfel'd module over $\mathbf{C}_{\infty}$. We denote it by $\mathfrak{a} * \phi$. For any two $\mathfrak{a}, \mathfrak{b} \in \mathfrak{I}_{\mathcal{O}}$, we have

$$
\mathfrak{a} *(\mathfrak{b} * \phi)=(\mathfrak{a} \mathfrak{b}) * \phi .
$$

Thus, $(\mathfrak{a}, \phi) \mapsto \mathfrak{a} * \phi$ is an action of $\mathfrak{I}_{\mathcal{O}}$ on the set of all Drinfel'd modules over $\mathbf{C}_{\infty}$.

Let $\mathfrak{F}_{\mathcal{O}}$ be the enveloping ("Grothendieck") group of the abelian semigroup $\mathfrak{I}_{\mathcal{O}}$. The abelian group $\mathfrak{F}_{\mathcal{O}}$ may be realized concretely as the group of fractional ideals of $k$ with respect to the Dedekind ring $\mathcal{O}$. One shows that the action of $\mathfrak{I}_{\mathcal{O}}$ on the set of Drinfel'd modules over $\mathbf{C}_{\infty}$ extends to an action of $\mathfrak{F} \mathcal{O}$. One also has the equality

$$
\phi_{\mathfrak{a} \mathfrak{b}}=(\mathfrak{b} * \phi)_{\mathfrak{a}} \phi_{\mathfrak{b}} \text {. }
$$

Torsion points. Let $\phi: \mathcal{O} \rightarrow \mathbf{C}_{\infty}\{\tau\}, a \mapsto \phi_{a}$, be a Drinfel'd module over $\mathbf{C}_{\infty}$. Remember that $\phi\left(\mathbf{C}_{\infty}\right)$ is the $\mathcal{O}$-module that is equal to $\mathbf{C}_{\infty}$ as an abelian group and whose $\mathcal{O}$-module structure is given by

$$
(a, x) \mapsto \phi_{a}(x) .
$$

Let $\phi\left(\mathbf{C}_{\infty}\right)^{\text {tor }}$ denote the $\mathcal{O}$-torsion submodule of $\phi\left(\mathbf{C}_{\infty}\right)$. In other words, an element $x \in \phi\left(\mathbf{C}_{\infty}\right)$ is in $\phi\left(\mathbf{C}_{\infty}\right)^{\text {tor }}$ if and only if $\phi_{a}(x)=0$ for some nonzero $a \in \mathcal{O}$.

For any $a \in \mathcal{O}$, let $\phi[a]=\operatorname{ker} \phi_{a}$. For any $a \in \mathfrak{I}_{\mathcal{O}}$, let $\phi[\mathfrak{a}]=\operatorname{ker} \phi_{\mathfrak{a}}$. Under the bijection given by equation (10) the sets $\phi\left(\mathbf{C}_{\infty}\right)^{\text {tor }}, \phi[a]$ and $\phi[a]$ are identified with the following subsets of $\mathbf{C}_{\infty} / L$ :

$$
\begin{aligned}
e_{L}^{-1}\left(\phi\left(\mathbf{C}_{\infty}\right)^{\text {tor }}\right) & =k L / L, \\
e_{L}^{-1}(\phi[a]) & =a^{-1} L / L \quad \text { for all } a \in \mathcal{O}-\{0\}, \\
e_{L}^{-1}(\phi[a]) & =a^{-1} L / L \quad \text { for all } a \in \widetilde{I}_{\mathcal{O}} .
\end{aligned}
$$


Here $a^{-1}$ is the inverse of $a$ as a fractional ideal with respect to $\mathcal{O}$, i.e.

$$
\mathfrak{a}^{-1}=\{x \in k \mid x \mathfrak{a} \subset \mathcal{O}\} .
$$

The following equalities follow from the definitions:

$$
\begin{aligned}
\phi[a] & =\phi[a \mathcal{O}] \quad \text { for all } a \in \mathcal{O}, \\
\phi[\mathfrak{a}] & =\bigcap_{a \in \mathfrak{a}} \phi[a] \quad \text { for all } a \in \mathfrak{I}_{\mathcal{O}}, \\
\phi\left(\mathbf{C}_{\infty}\right)^{\text {tor }} & =\bigcup_{a \in \mathcal{O}} \phi[a], \\
\phi\left(\mathbf{C}_{\infty}\right)^{\text {tor }} & =\bigcup_{\mathfrak{a} \in \mathfrak{I}_{\mathcal{O}}} \phi[\mathfrak{a}],
\end{aligned}
$$

and

$$
\mathfrak{a} \mid \mathfrak{b} \Longleftrightarrow \phi[\mathfrak{a}] \subset \phi[\mathfrak{b}] \quad \text { for all } \mathfrak{a}, \mathfrak{b} \in \mathfrak{I}_{\mathcal{O}} .
$$

One also checks that, for all $\mathfrak{a}, \mathfrak{b} \in \mathfrak{I}_{\mathcal{O}}$,

$$
\begin{aligned}
& \phi[\mathfrak{a}] \cap \phi[\mathfrak{b}]=\phi[\mathfrak{a}+\mathfrak{b}], \\
& \phi[\mathfrak{a}]+\phi[\mathfrak{b}]=\phi[\mathfrak{a} \cap \mathfrak{b}] .
\end{aligned}
$$

We have

$$
\operatorname{Card} \phi[\mathfrak{a}]=(\mathbf{N} a)^{r} \quad \text { for all } a \in \mathfrak{I}_{\mathcal{O}},
$$

where $r$ is the rank of $\phi$ and $\mathbf{N a}$ is the absolute norm of $a$, i.e., $\mathbf{N a}$ is the cardinal of $\mathcal{O} / \mathfrak{a}$.

Let $a \in \mathfrak{I}_{\mathcal{O}}$. By construction, $\phi_{\mathfrak{a}}$ is an $\mathcal{O}$-module morphism

$$
\phi_{a}: \phi\left(\mathbf{C}_{\infty}\right) \rightarrow(a * \phi)\left(\mathbf{C}_{\infty}\right) .
$$

For any $\mathfrak{b} \in \mathfrak{I}_{\mathcal{O}}$, let $\left.\phi_{\mathfrak{a}}\right|_{\phi[\mathfrak{b}]}$ denote the restriction of $\phi_{\mathfrak{a}}$ to $\phi[\mathfrak{b}]$.

Lemma 1.2.4. Let $\phi$ be a Drinfel'd module over $\mathbf{C}_{\infty}$. Let $\mathfrak{a}, \mathfrak{b} \in \mathfrak{I}_{\mathcal{O}}$. Let $\mathfrak{b}=\mathfrak{a}+\mathfrak{b}$ be the gcd of $\mathfrak{a}$ and $\mathfrak{b}$. We have

$$
\begin{aligned}
\operatorname{Ker}\left(\left.\phi_{\mathfrak{a}}\right|_{\phi[\mathfrak{b}]}\right) & =\phi[\delta], \\
\operatorname{Im}\left(\left.\phi_{\mathfrak{a}}\right|_{\phi[\mathfrak{b}]}\right) & =(\mathfrak{a} * \phi)\left[\mathfrak{D}^{-1} \mathfrak{b}\right] .
\end{aligned}
$$

Proof. First equality: we have $\operatorname{Ker}\left(\left.\phi_{\mathfrak{a}}\right|_{\phi[\mathfrak{b}]}\right)=\phi[\mathfrak{a}] \cap \phi[\mathfrak{b}]$, so the result follows from equation (15).

Second equality: let $r$ denote the rank of $\phi$. We have

$$
\begin{aligned}
\operatorname{Card}\left(\operatorname{Im}\left(\left.\phi_{\mathfrak{a}}\right|_{\phi[\mathfrak{b}]}\right)\right) & =\operatorname{Card}(\phi[\mathfrak{b}]) / \operatorname{Card}\left(\operatorname{Ker}\left(\left.\phi_{\mathfrak{a}}\right|_{\phi[\mathfrak{b}]}\right)\right) \\
& =\operatorname{Card}(\phi[\mathfrak{b}]) / \operatorname{Card}(\phi[\mathfrak{b}]) \\
& =(\mathbf{N b})^{r} /(\mathbf{N} \mathfrak{b})^{r}
\end{aligned}
$$


and

$$
\operatorname{Card}\left((\mathfrak{a} * \phi)\left[\mathfrak{D}^{-1} \mathfrak{b}\right]\right)=\mathbf{N}\left(\mathfrak{D}^{-1} \mathfrak{b}\right)^{r},
$$

so the two cardinals are equal, so it is enough to show one inclusion. Let $x \in$ $\operatorname{Im}\left(\left.\phi_{\mathfrak{a}}\right|_{\phi[\mathfrak{b}]}\right)$. It is enough to show that $(\mathfrak{a} * \phi)_{\mathfrak{b}}^{-1_{\mathfrak{b}}}(x)=0$. Let $y \in \phi[\mathfrak{b}]$ such that $\phi_{\mathfrak{a}}(y)=x$. Let $\mathfrak{c}=\mathfrak{a} \cap \mathfrak{b}$ be the $1 \mathrm{~cm}$. We have $\mathfrak{b}^{-1} \mathfrak{b}=\mathfrak{a}^{-1} \mathfrak{c}$. But

$$
(a * \phi)_{\mathfrak{a}^{-1} \mathfrak{c}}(x)=(\mathfrak{a} * \phi)_{\mathfrak{a}^{-1} \mathfrak{c}}\left(\phi_{\mathfrak{a}}(y)\right),
$$

so,by equation (13), we get

$$
(a * \phi)_{\mathfrak{a}^{-1} \mathfrak{c}}(x)=\phi_{\mathfrak{c}}(y) .
$$

But $\mathfrak{b} \mid \mathrm{c}$ and $y \in \phi[\mathfrak{b}]$, so $y \in \phi[\mathfrak{c}]$, so

$$
(\mathfrak{a} * \phi)_{\mathfrak{a}^{-1} \mathfrak{c}}(x)=0 .
$$

Corollary 1.2.5. Let $\phi$ be a Drinfel'd module over $\mathbf{C}_{\infty}$. Let $\mathfrak{a}, \mathfrak{b} \in \mathfrak{I}_{\mathcal{O}}$. For all $\lambda \in \phi[\mathfrak{b}]$, there exists $\mu \in\left(\mathfrak{a}^{-1} * \phi\right)[\mathfrak{a} \mathfrak{b}]$ such that

$$
\left(\mathfrak{a}^{-1} * \phi\right)_{\mathfrak{a}}(\mu)=\lambda \text {. }
$$

Proof. Let $\psi=\mathfrak{a}^{-1} * \phi$. Let $\mathfrak{b}_{2}=a \mathfrak{b}$. Let $\delta_{2}=\mathfrak{a}$, so that $\delta_{2}$ is the gcd of $a$ and $\mathfrak{b}_{2}$. By Lemma 1.2.4, we have

$$
\operatorname{Im}\left(\left.\psi_{\mathfrak{a}}\right|_{\psi\left[\mathfrak{b}_{2}\right]}\right)=\phi\left[\delta_{2}^{-1} \mathfrak{b}_{2}\right],
$$

so

$$
\operatorname{Im}\left(\left.\psi_{\mathfrak{a}}\right|_{\psi[\mathfrak{a} b]}\right)=\phi[\mathfrak{b}] .
$$

Corollary 1.2.6. Let $\phi$ be a Drinfel'd module over $\mathbf{C}_{\infty}$. For all $a \in \mathfrak{I}_{\mathcal{O}}$, the map

$$
\left(\mathfrak{a}^{-1} * \phi\right)_{\mathfrak{a}}:\left(\mathfrak{a}^{-1} * \phi\right)\left(\mathbf{C}_{\infty}\right)^{\text {tor }} \rightarrow \phi\left(\mathbf{C}_{\infty}\right)^{\text {tor }}
$$

is surjective.

1.3. Hayes' explicit class field theory. In this subsection we review D. R. Hayes' explicit class field theory for function fields, in terms of sign-normalized rank one Drinfel'd modules. We follow [20], Part II, and [15], Chapter VII. Recall that $k_{\infty}$ is the completion of $k$ at $\infty$. Let $\mathbb{F}_{\infty}$ denote the constant subfield of $k_{\infty}$. The field $\mathbb{F}_{\infty}$ is a finite extension of $\mathbb{F}_{q}$, and its degree is equal to the degree of the place $\infty$.

Definition 1.3.1. A sign function on $k_{\infty}^{*}$ is a group morphism sgn: $k_{\infty}^{*} \rightarrow \mathbb{E}_{\infty}^{*}$ which induces the identity map on $\mathbb{F}_{\infty}^{*}$. 
Let us choose once and for all a sign-function sgn (by [20], Corollary 12.2, the number of possible choices is equal to the cardinal of $\mathbb{F}_{\infty}^{*}$ ). We let $\operatorname{sgn}(0)=0$ so that sgn becomes a function $k_{\infty} \rightarrow \mathbb{F}_{\infty}$.

Definition 1.3.2. A Drinfel'd module $\phi$ over $\mathbf{C}_{\infty}$ is said to be sgn-normalized if there exists an element $\sigma \in \operatorname{Gal}\left(\mathbb{F}_{\infty} / \mathbb{F}_{q}\right)$ such that

$$
\mu_{\phi}(a)=\sigma(\operatorname{sgn}(a)) \quad \text { for all } a \in \mathcal{O} .
$$

Let us now focus on the case of Drinfel'd modules of rank one.

Definition 1.3.3. Let $H(\operatorname{sgn})$ denote the set of sgn-normalized rank one Drinfel'd modules over $\mathbf{C}_{\infty}$. The elements of $H(\mathrm{sgn})$ are also called Hayes modules (for the triple $(k, \infty, \operatorname{sgn}))$.

Proposition 1.3.4. $H(\mathrm{sgn})$ is a finite set, and its cardinal $h(\mathrm{sgn})$ is given by

$$
h(\operatorname{sgn})=\frac{\operatorname{Card} \mathbb{F}_{\infty}^{*}}{\operatorname{Card} \mathbb{F}_{q}^{*}} \cdot h(\mathcal{O}),
$$

where $h(\mathcal{O})$ is the class number of the Dedekind ring $\mathcal{O}$.

Proof. See [20], Corollary 13.4.

Proposition 1.3.5. For any $\phi \in H(\mathrm{sgn})$ and any $a \in \mathfrak{F}_{\mathcal{O}}$, we have $a * \phi \in H(\mathrm{sgn})$. Thus, $\mathfrak{F} \mathcal{O}$ acts on $H(\mathrm{sgn})$.

Proof. See [20], p. 22.

Definition 1.3.6. Let $\phi \in H(\operatorname{sgn})$, and let $y \in \mathcal{O}-\mathbb{F}_{q}$ (recall that $\mathbb{F}_{q}$ denotes the constant subfield of $k$ ). Let $H^{+}$be the field generated over $k$ by the coefficients of $\phi_{y}$.

One shows (see [20], p. 23) that $H^{+}$does not depend on the choice of $\phi$ and $y$.

Proposition 1.3.7. The extension $H^{+} / k$ is finite, abelian, and unramified away from $\infty$.

Proof. See [20], Propositions 14.1 and 14.4.

One shows (see [20], §15) that $H^{+}$contains a subfield $H$ which plays the rôle of the Hilbert class field for the pair $(k, \infty)$.

Here is a concrete picture of the Galois group $\operatorname{Gal}\left(H^{+} / k\right)$. First, let $\mathcal{P}_{\mathcal{O}}^{+}$be the following subgroup of $\mathfrak{F}_{\mathcal{O}}$ :

$$
\mathcal{P}_{\mathcal{O}}^{+}=\{x \mathcal{O} \mid x \in k, \operatorname{sgn}(x)=1\} .
$$

We then have the following proposition. 
Proposition 1.3.8. The Artin map $\left(\cdot, H^{+} / k\right)$ induces an isomorphism from $\mathfrak{F}_{\mathcal{O}} / \mathcal{P}_{\mathcal{O}}^{+}$to $\operatorname{Gal}\left(H^{+} / k\right)$.

Proof. See [20], Theorem 14.7.

The Galois group $\operatorname{Gal}\left(H^{+} / k\right)$ acts on $H(\operatorname{sgn})$ by $(\sigma, \phi) \mapsto \sigma \phi$, where $\sigma \phi$ is defined by $(\sigma \phi)_{a}=\sigma\left(\phi_{a}\right)$ for all $a \in \mathcal{O}$ (one checks that $\sigma \psi \in H(\operatorname{sgn})$ ).

Theorem 1.3.9. For any $a \in \mathfrak{I}_{\mathcal{O}}$, if $\sigma_{\mathfrak{a}}=\left(\mathfrak{a}, H^{+} / k\right) \in \operatorname{Gal}\left(H^{+} / k\right)$ denotes the Artin automorphism of $\mathrm{H}^{+}$associated to a, then we have

$$
\sigma_{a} \phi=a * \phi \quad \text { for all } \phi \in H(\text { sgn }) .
$$

The set $H(\mathrm{sgn})$ is principal homogeneous under the action of $\mathrm{Gal}\left(H^{+} / k\right)$.

Proof. See [20], Theorems 13.8 and 14.7.

Definition 1.3.10. For any $\phi \in H(\operatorname{sgn})$, let $K$ denote the field generated over $H^{+}$ by the elements of $\phi\left(\mathbf{C}_{\infty}\right)^{\text {tor }}$. For any $c \in \mathfrak{I}_{\mathcal{O}}$, let $K_{\mathfrak{c}}$ denote the field generated over $H^{+}$by the elements of $\phi[\mathrm{c}]$.

One shows (see [20], p. 28) that $K$ and $K_{\mathfrak{c}}$ are independent of the choice of $\phi$. The extension $K_{\mathfrak{c}} / k$ is called the narrow ray class extension modulo c. By construction, we have

$$
K=\bigcup_{\mathfrak{c} \in \mathfrak{I}_{\mathcal{O}}} K_{\mathfrak{c}} .
$$

Theorem 1.3.11. For any $\mathrm{c} \in \mathfrak{I}_{\mathcal{O}}$, the extension $K_{\mathfrak{c}} / k$ is finite, abelian, and unramified away from $\infty$ and the prime divisors of $c$. Moreover, $K_{\mathfrak{c}}$ contains the ray class field of $k$ of conductor $c$ totally split at $\infty$. For any a $\in \mathfrak{I}_{\mathcal{O}}$ prime to $c$, if $\sigma_{\mathfrak{a}}=\left(\mathfrak{a}, K_{\mathfrak{c}} / k\right) \in \operatorname{Gal}\left(K_{\mathfrak{c}} / k\right)$ denotes the Artin automorphism of $K_{\mathfrak{c}}$ associated to a, then we have

$$
\sigma_{\mathfrak{a}} \lambda=\phi_{\mathfrak{a}}(\lambda) \text { for all } \phi \in H(\operatorname{sgn}), \lambda \in \phi[c] .
$$

Proof. See [20], p. 28, or [19], Section 8.

In particular, this shows that

$$
k^{\mathrm{ab}, \infty} \subset K \subset k^{\mathrm{ab}},
$$

where $k^{\mathrm{ab}, \infty}$ is the maximal abelian extension of $k$ that is totally split at $\infty$.

Let us give a concrete picture of the $\operatorname{Galois}$ group $\operatorname{Gal}\left(K_{\mathfrak{c}} / k\right)$, for $\mathfrak{c} \in \mathfrak{I}_{\mathcal{O}}$. Let $\mathfrak{F}_{\mathcal{O}}$ (c) denote the subgroup of $\mathfrak{F}_{\mathcal{O}}$ of all fractional ideals that are prime to $c$, and let

$$
\mathcal{P}_{\mathcal{O}}^{+}(\mathfrak{c})=\{x \mathcal{O} \mid x \in k, \operatorname{sgn}(x)=1, x \equiv 1 \bmod c\} .
$$

We then have the following proposition. 
Proposition 1.3.12. The Artin map $\left(\cdot, K_{\mathfrak{c}} / k\right)$ induces an isomorphism from $\mathfrak{F}_{\mathcal{O}}(\mathrm{c}) / \mathcal{P}_{\mathcal{O}}^{+}(\mathrm{c})$ to $\operatorname{Gal}\left(K_{\mathrm{c}} / k\right)$.

Proof. See [20], p. 28.

Moreover, the Galois group $\operatorname{Gal}\left(K_{\mathfrak{c}} / H^{+}\right)$has an even simpler description: one can check (loc. cit.) that it is isomorphic to the group of invertible elements in $\mathcal{O} / \mathrm{c}$.

\section{Construction of the $C^{*}$-dynamical system $\left(C_{k, \infty},\left(\sigma_{t}\right)\right)$}

2.1. The space $X$ of characters. For any $\phi \in H(\operatorname{sgn})$, let $X_{\phi}$ be the dual group of the discrete abelian torsion group $\phi\left(\mathbf{C}_{\infty}\right)^{\text {tor }}$. Thus, an element of $X_{\phi}$ is a character of $\phi\left(\mathbf{C}_{\infty}\right)^{\text {tor }}$. The group $X_{\phi}$ is profinite,

$$
X_{\phi}=\lim _{\leftarrow a} \widehat{\phi[a]}
$$

where a runs over $\mathfrak{I}_{\mathcal{O}}$ ordered by divisibility. Let $X$ be the (disjoint) union of the $X_{\phi}$,

$$
X=\bigcup_{\phi \in H(\operatorname{sgn})} X_{\phi}
$$

Note that the elements of $X$ are reminiscent of characters in [17] and of $\mathbb{Q}$-lattices (or $k$-lattices) in [11] and [12], [13].

Lemma 2.1.1. For any character $\chi \in X$, we have

$$
\operatorname{Im} \chi \subset \mathbb{U}_{p}
$$

where $\mathbb{U}_{p}$ is the group of $p$-th roots of unity in $\mathbb{C}$.

Proof. Recall that for any $\phi \in H(\operatorname{sgn})$, as a group, $\phi\left(\mathbf{C}_{\infty}\right)$ is equal to $\mathbf{C}_{\infty}$, which is a field of characteristic $p$. Thus, for all $\lambda \in \phi\left(\mathbf{C}_{\infty}\right)^{\text {tor }}$, we have $\chi(\lambda)^{p}=\chi(p \lambda)=$ $\chi(0)=1$.

Lemma 2.1.2. $X$ is compact (and Hausdorff).

Proof. For any $\phi \in H(\mathrm{sgn})$, the group $X_{\phi}$ is profinite, hence compact. As $H(\operatorname{sgn})$ is finite, $X$ is compact.

We define an action of $\mathfrak{s}_{\mathcal{O}}$ on $X$ by

$$
\chi^{\mathfrak{a}}=\chi \circ\left(\mathfrak{a}^{-1} * \phi\right)_{\mathfrak{a}} \quad \text { for all } a \in \mathfrak{s}_{\mathcal{O}}, \phi \in H(\operatorname{sgn}), \chi \in X_{\phi}
$$


Recall that $\left(\mathfrak{a}^{-1} * \phi\right)_{\mathfrak{a}}$ is a map from $\left(\mathfrak{a}^{-1} * \phi\right)\left(\mathbf{C}_{\infty}\right)$ to $\phi\left(\mathbf{C}_{\infty}\right)$. Thus, if $\chi \in X_{\phi}$ then $\chi^{\mathfrak{a}} \in X_{\mathfrak{a}^{-1} * \phi}$. Note that equation (13) guarantees that this is a semigroup action of $\mathfrak{I}_{\mathcal{O}}$.

The exponent notation $\left(\chi^{\mathfrak{a}}\right)$ is inspired by what happens with characters of $\mathbb{Q} / \mathbb{Z}$. These characters may be composed with the map $\phi_{n}: x \mapsto n x$, for any $n \in \mathbb{N}^{*}$. By definition of a character, we have $\chi \circ \phi_{n}=\chi^{n}$. In our case $\mathbb{N}^{*}$ is replaced by $\mathfrak{I}_{\mathcal{O}}$ and the maps $\phi_{n}$ are replaced by the $\phi_{\mathfrak{a}}$.

We define an action of $\operatorname{Gal}(K / k)$ on $X$ by

$$
\sigma \chi=\chi \circ \sigma \quad \text { for all } \sigma \in \operatorname{Gal}(K / k), \chi \in X \text {. }
$$

One checks that the actions of $\operatorname{Gal}(K / k)$ and of $\mathfrak{I}_{\mathcal{O}}$ on $X$ commute with one another.

Lemma 2.1.3. For all $a \in \mathfrak{I}_{\mathcal{O}}$, the map $X \rightarrow X, \chi \mapsto \chi^{\mathfrak{a}}$, is injective.

Proof. Let $\chi_{1}, \chi_{2} \in X$ such that $\chi_{1}^{a}=\chi_{2}^{a}$. For $i=1,2$ let $\phi^{i}$ be such that $\chi_{i} \in X_{\phi^{i}}$. By definition, we have $\chi_{i}^{\mathfrak{a}} \in X_{\mathfrak{a}^{-1} * \phi^{i}}$, so $a^{-1} * \phi^{1}=a^{-1} * \phi^{2}$, so $\phi^{1}=\phi^{2}$. Let $\phi=\phi^{1}=\phi^{2}$. We have

$$
\chi_{1} \circ\left(\mathfrak{a}^{-1} * \phi\right)_{\mathfrak{a}}=\chi_{2} \circ\left(\mathfrak{a}^{-1} * \phi\right)_{\mathfrak{a}} .
$$

Corollary 1.2.6 then shows that $\chi_{1}=\chi_{2}$.

Corollary 2.1.4. Let $\mathfrak{a}_{1}, \mathfrak{a}_{2}, \mathfrak{b}_{1}, \mathfrak{b}_{2} \in \mathfrak{I}_{\mathcal{O}}$ be such that $\mathfrak{a}_{1}^{-1} \mathfrak{a}_{2}=\mathfrak{b}_{1}^{-1} \mathfrak{b}_{2}$.

(1) Let $\chi_{1}, \chi_{2} \in X$. We have

$$
\chi_{1}^{\mathfrak{a}_{1}}=\chi_{2}^{\mathfrak{a}_{2}} \Longleftrightarrow \chi_{1}^{\mathfrak{b}_{1}}=\chi_{2}^{\mathfrak{b}_{2}} .
$$

(2) Let $\chi_{1}, \chi_{2}, \chi_{3} \in X$. We have

$$
\chi_{1}^{\mathfrak{a}_{1}}=\chi_{2}^{\mathfrak{a}_{2}} \text { and } \chi_{3}^{\mathfrak{b}_{1}}=\chi_{2}^{\mathfrak{b}_{2}} \Longrightarrow \chi_{1}=\chi_{3} .
$$

Proof. Let us first prove (1). Suppose that $\chi_{1}^{\alpha_{1}}=\chi_{2}^{\alpha_{2}}$. We have $\chi_{1}^{\alpha_{1} \mathfrak{b}_{2}}=\chi_{2}^{\alpha_{2} \mathfrak{b}_{2}}$. But $\mathfrak{a}_{2} \mathfrak{b}_{1}=a_{1} \mathfrak{b}_{2}$, so $\chi_{1}^{\mathfrak{a}_{2} \mathfrak{b}_{1}}=\chi_{2}^{\mathfrak{a}_{2} \mathfrak{b}_{2}}$, so

$$
\left(\chi_{1}^{\mathfrak{b}_{1}}\right)^{\mathfrak{a}_{2}}=\left(\chi_{2}^{\mathfrak{b}_{2}}\right)^{\mathfrak{a}_{2}},
$$

so Lemma 2.1.3 gives $\chi_{1}^{\mathfrak{b}_{1}}=\chi_{2}^{\mathfrak{b}_{2}}$, which proves one implication, and the other implication follows by swapping $\mathfrak{a}_{i}$ with $\mathfrak{b}_{i}$ for $i=1,2$.

Let us now prove (2). We have

$$
\chi_{1}^{\mathfrak{a}_{1} \mathfrak{b}_{1}}=\chi_{2}^{\mathfrak{a}_{2} \mathfrak{b}_{1}}=\chi_{2}^{a_{1} \mathfrak{b}_{2}}=\chi_{3}^{a_{1} \mathfrak{b}_{1}},
$$

so Lemma 2.1.3 gives $\chi_{1}=\chi_{3}$. 
Corollary 2.1.4 allows to extend the action of $\mathfrak{S}_{\mathcal{O}}$ on $X$ to a partially defined action of $\mathfrak{F} \mathcal{O}$ as follows.

Definition 2.1.5. For any $\chi \in X$, let $\mathfrak{F}_{\chi}$ denote the set of all $c \in \mathfrak{F}_{\mathcal{O}}$ such that there exists $\chi_{1} \in X$ satisfying

$$
\chi_{1}^{a_{1}}=\chi^{a_{2}}
$$

for some $a_{1}, a_{2} \in \mathfrak{I}_{\mathcal{O}}$ with $c=a_{1}^{-1} a_{2}$. By Corollary 2.1.4(1), the existence of $\chi_{1}$ only depends on $\chi$ and $c$, and does not depend on the choice of $a_{1}, a_{2} \in \mathfrak{s}_{\mathcal{O}}$ such that $c=a_{1}^{-1} a_{2}$. By Corollary 2.1.4 (2), the character $\chi_{1}$, when it exists, is uniquely determined by $\chi$ and $c$. When $c \in \mathfrak{F}_{\chi}$, we define a character $\chi^{\mathfrak{c}}$ by

$$
\chi^{\mathfrak{c}}=\chi_{1} \text {. }
$$

The partially defined map $\mathfrak{F}_{\mathcal{O}} \times X \rightarrow X,(\mathfrak{c}, \chi) \mapsto \chi^{\mathfrak{c}}$, should be regarded as a partially defined group action of $\mathfrak{F}_{\mathcal{O}}$ on $X$. For any $(\mathfrak{c}, \chi)$, the character $\chi^{\mathfrak{c}}$ is defined if and only if $\mathfrak{c} \in \mathfrak{F}_{\chi}$. For any $\mathfrak{c}_{1}, \mathfrak{c}_{2}$ in $\mathfrak{F}_{\chi}$, if $\mathfrak{c}_{1} \mathfrak{c}_{2} \in \mathfrak{F}_{\chi}$ one checks that $\chi^{\mathfrak{c}_{1} \mathfrak{c}_{2}}=\left(\chi^{\mathfrak{c}_{1}}\right)^{\mathfrak{c}_{2}}$. Of course, when $\mathfrak{c} \in \mathfrak{S}_{\mathcal{O}}$ the character $\chi^{\mathfrak{c}}$ is just the one that was defined in equation (18).

For any $\chi$, we have $\mathfrak{I}_{\mathcal{O}} \subset \mathfrak{F}_{\chi}$. Characters $\chi \in X$ for which this inclusion is an equality $\left(\mathfrak{F}_{\chi}=\mathfrak{I}_{\mathcal{O}}\right)$ will be called admissible, and will play an important rôle later (see Subsection 3.6).

Note that we obviously have

$$
\mathfrak{F}_{\chi^{a}}=a^{-1} \mathfrak{F}_{\chi} \quad \text { for all } \chi \in X, a \in \mathfrak{I}_{\mathcal{O}}
$$

Lemma 2.1.6. Let $\chi \in X$. Let $\phi \in H(\mathrm{sgn})$ such that $\chi \in X_{\phi}$. For any a $\in \mathfrak{s}_{\mathcal{O}}$, we have

$$
a^{-1} \in \mathfrak{F}_{\chi} \Longleftrightarrow \chi(\lambda)=1 \text { for all } \lambda \in \phi[a] .
$$

When this is the case, the character $\chi^{a^{-1}}$ is given by

$$
\chi^{a^{-1}}(\lambda)=(\mathbf{N a})^{-1} \sum_{\phi_{a}(\mu)=\lambda} \chi(\mu) \text { for all } \lambda \in(a * \phi)\left(\mathbf{C}_{\infty}\right)^{\text {tor }} .
$$

Proof. If $a^{-1} \in \mathfrak{F}_{\chi}$, then there exists $\chi_{1} \in X$ such that $\chi=\chi_{1}^{a}$. Thus, for all $\lambda \in \phi[a]$, we have $\chi(\lambda)=\chi_{1}\left(\phi_{a}(\lambda)\right)$, but $\phi_{a}(\lambda)=0$, so $\chi(\lambda)=1$.

Now suppose that for all $\lambda \in \phi[a], \chi(\lambda)=1$. For all $\lambda \in(a * \phi)\left(\mathbf{C}_{\infty}\right)^{\text {tor }}$, set

$$
\chi_{1}(\lambda)=(\mathbf{N a})^{-1} \sum_{\phi_{a}(\mu)=\lambda} \chi(\mu) .
$$

Let us show that this defines a character $\chi_{1}$ of $(a * \phi)\left(\mathbf{C}_{\infty}\right)^{\text {tor }}$. Let $\lambda \in(a * \phi)\left(\mathbf{C}_{\infty}\right)^{\text {tor }}$. By Lemma 1.2.6, there exists $\mu_{1} \in \phi\left(\mathbf{C}_{\infty}\right)^{\text {tor }}$ such that $\phi_{\mathfrak{a}}\left(\mu_{1}\right)=\lambda$. We have

$$
\chi_{1}(\lambda)=(\mathbf{N a})^{-1} \sum_{\mu_{0} \in \phi[a]} \chi\left(\mu_{0}+\mu_{1}\right)=(\mathbf{N a})^{-1}\left(\sum_{\mu_{0} \in \phi[a]} \chi\left(\mu_{0}\right)\right) \chi\left(\mu_{1}\right) .
$$


But we have $\chi\left(\mu_{0}\right)=1$ for all $\mu_{0} \in \phi[a]$, and by equation (17) we have that $\operatorname{Card}(\phi[a])=\mathbf{N a}$. Thus, we get

$$
\chi_{1}(\lambda)=\chi\left(\mu_{1}\right) \text { for all } \lambda \in(a * \phi)\left(\mathbf{C}_{\infty}\right)^{\text {tor }} \text { and for all } \mu_{1} \text { with } \phi_{a}\left(\mu_{1}\right)=\lambda .
$$

Now let $\lambda^{\prime} \in(a * \phi)\left(\mathbf{C}_{\infty}\right)^{\text {tor }}$ and $\mu_{1}^{\prime}$ such that $\phi_{a}\left(\mu_{1}^{\prime}\right)=\lambda^{\prime}$. We have

$$
\lambda+\lambda^{\prime}=\phi_{a}\left(\mu_{1}\right)+\phi_{a}\left(\mu_{1}^{\prime}\right)=\phi_{a}\left(\mu_{1}+\mu_{1}^{\prime}\right),
$$

hence

$$
\chi_{1}\left(\lambda+\lambda^{\prime}\right)=\chi_{1}\left(\phi_{a}\left(\mu_{1}+\mu_{1}^{\prime}\right)\right)=\chi\left(\mu_{1}+\mu_{1}^{\prime}\right)=\chi\left(\mu_{1}\right) \chi\left(\mu_{1}^{\prime}\right)
$$

so

$$
\chi_{1}\left(\lambda+\lambda^{\prime}\right)=\chi_{1}(\lambda) \chi_{1}\left(\lambda^{\prime}\right)
$$

which implies $\chi_{1}(\lambda)^{p}=\chi_{1}(p \lambda)=\chi_{1}(0)=1$, i.e.,

$$
\chi_{1}(\lambda) \in \mathbb{U}_{p} \quad \text { for all } \lambda \in(a * \phi)\left(\mathbf{C}_{\infty}\right)^{\text {tor }} .
$$

Hence $\chi_{1}$ is a group morphism $(a * \phi)\left(\mathbf{C}_{\infty}\right)^{\text {tor }} \rightarrow \mathbb{U}_{p}$, so $\chi_{1} \in X$, and we have by construction $\chi_{1}^{\mathfrak{a}}=\chi$. Thus, we have $\mathfrak{a}^{-1} \in \mathfrak{F}_{\chi}$ and $\chi^{\mathfrak{a}^{-1}}=\chi_{1}$.

Lemma 2.1.7. For all $\chi \in X$, for all $\mathfrak{a}, \mathfrak{b} \in \mathfrak{s}_{\mathcal{O}}$ relatively prime, we have

$$
a^{-1} \mathfrak{b} \in \mathfrak{F}_{\chi} \Longleftrightarrow a^{-1} \in \mathfrak{F}_{\chi}
$$

Proof. Let $\phi$ be such that $\chi \in X_{\phi}$. We have $a^{-1} \mathfrak{b} \in \mathfrak{F}_{\chi} \Leftrightarrow \mathfrak{a}^{-1} \in \mathfrak{F}_{\chi^{\mathfrak{b}}}$. Lemma 2.1.6 applied to $\chi^{\mathfrak{b}}$ thus gives

$$
\mathfrak{a}^{-1} \mathfrak{b} \in \mathfrak{F}_{\chi} \Longleftrightarrow \chi\left(\left(\mathfrak{b}^{-1} * \phi\right)_{\mathfrak{b}}(\lambda)\right)=1 \quad \text { for all } \lambda \in\left(\mathfrak{b}^{-1} * \phi\right)[\mathfrak{a}] .
$$

But, as $\mathfrak{a}$ and $\mathfrak{b}$ are relatively prime, by Lemma 1.2 .4 , the map $\lambda \mapsto \mathfrak{b} \lambda$ is a bijection from $\left(\mathfrak{b}^{-1} * \phi\right)[\mathfrak{a}]$ onto $\phi[\mathfrak{a}]$. Thus we get

$$
\mathfrak{a}^{-1} \mathfrak{b} \in \mathfrak{F}_{\chi} \Longleftrightarrow \chi(\lambda)=1 \quad \text { for all } \lambda \in \phi[\mathfrak{a}],
$$

and, by Lemma 2.1.6, this is equivalent to $a^{-1} \in \mathfrak{F}_{\chi}$.

Lemma 2.1.8. For all $\chi \in X$, for all $\mathfrak{a}, \mathfrak{b} \in \mathfrak{s}_{\mathcal{O}}$ relatively prime, we have

$$
(\mathfrak{a b})^{-1} \in \mathfrak{F}_{\chi} \Longleftrightarrow \mathfrak{a}^{-1} \in \mathfrak{F}_{\chi} \text { and } \mathfrak{b}^{-1} \in \mathfrak{F}_{\chi}
$$

Proof. Let $\phi$ be such that $\chi \in X_{\phi}$. By Lemma 2.1.6, the statement that we want to prove is equivalent to the following:

$$
\chi(\lambda)=1 \quad \text { for all } \lambda \in \phi[\mathfrak{a} \mathfrak{b}] \Longleftrightarrow \begin{array}{ll}
\chi(\lambda)=1 & \text { for all } \lambda \in \phi[\mathfrak{a}], \\
\chi(\lambda)=1 & \text { for all } \lambda \in \phi[\mathfrak{b}] .
\end{array}
$$


By equations (15) and (16), as $\mathfrak{a}$ and $\mathfrak{b}$ are relatively prime, we have

$$
\phi[\mathfrak{a} \mathfrak{b}]=\phi[\mathfrak{a}] \oplus \phi[\mathfrak{b}],
$$

so, for any $\lambda \in \phi[\mathfrak{a} \mathfrak{b}]$, there exists a unique pair $\left(\lambda_{1}, \lambda_{2}\right) \in \phi[\mathfrak{a}] \times \phi[\mathfrak{b}]$ such that $\lambda=\lambda_{1}+\lambda_{2}$. We have $\chi(\lambda)=\chi\left(\lambda_{1}\right) \chi\left(\lambda_{2}\right)$, so equation (22) follows.

2.2. Construction of the groupoid $\mathscr{E}$ and of the dynamical system $\left(C_{k, \infty},\left(\sigma_{t}\right)\right)$. Let $\mathcal{G}$ be the following subset of $X \times \mathfrak{F} \mathcal{O}$ :

$$
\mathscr{E}=\left\{(\chi, c) \in X \times \mathfrak{F}_{\mathcal{O}} \mid \mathfrak{c} \in \mathfrak{F}_{\chi}\right\}
$$

We turn $\mathcal{E}$ into a groupoid by endowing it with the groupoid law

$$
\left(\chi_{1}, c_{1}\right) \circ\left(\chi_{2}, c_{2}\right)=\left(\chi_{2}, c_{1} c_{2}\right) \text { if } \chi_{1}=\chi_{2}^{c_{2}}
$$

and the inverse map

$$
(\chi, c)^{-1}=\left(\chi^{c}, c^{-1}\right) .
$$

One checks that, under the identification $\mathscr{E}^{(0)}=X \times\{1\} \simeq X$, the range and source maps $r$ and $s$ are respectively given by $r(\chi, c)=\chi^{\mathfrak{c}}$ and $s(\chi, c)=\chi$.

The abelian group $\mathfrak{F}_{\mathcal{O}}$ is endowed with the discrete topology. The groupoid $\mathscr{E}$ is endowed with its topology as a subset of $X \times \mathfrak{F} \mathcal{O}$.

Lemma 2.2.1. $\mathcal{E}$ is a locally compact groupoid.

Proof. $X \times \mathfrak{F}_{\mathcal{O}}$ is locally compact by Lemma 2.1 .2 and $\mathcal{E}$ is a closed subset of it, so it is also locally compact. It is clear that the composition and inverse maps are continuous, so this is a locally compact groupoid.

The $\mathrm{C}^{*}$-algebra $C_{k, \infty}$ that was advertised in the introduction of this paper is the maximal ${ }^{2} \mathrm{C}^{*}$-algebra of the groupoid $\mathscr{G}$. Let us quickly explain what that means.

For $\chi \in X$, let $E_{\chi}$ denote the fiber of $s$ above $\chi$, that is,

$$
\mathscr{E}_{\chi}=\{\chi\} \times \mathfrak{F}_{\chi},
$$

so $\mathscr{G}_{\chi}$ is discrete and is in bijection with $\mathfrak{F}_{\chi}$.

Let $C_{c}(\mathscr{E})$ denote the convolution algebra of continuous maps $\mathcal{G} \rightarrow \mathbb{C}$ with compact support, where the convolution product is given by

$$
\left(f_{1} f_{2}\right)(g)=\sum_{g_{1} \circ g_{2}=g} f_{1}\left(g_{1}\right) f_{2}\left(g_{2}\right) .
$$

\footnotetext{
${ }^{2}$ Actually, it coincides with the reduced $C^{*}$-algebra because $\mathfrak{F}_{\mathcal{O}}$ is an abelian group, but we shall not need that in this paper.
} 
$C_{c}(\mathscr{E})$ is endowed with the involution $f \mapsto f^{*}$ defined by

$$
f^{*}(g)=\overline{f\left(g^{-1}\right)} .
$$

For any $\chi \in X$, we define a $*$-representation of $C_{c}(\mathscr{E})$ on the Hilbert space $\ell^{2}\left(\mathscr{E}_{\chi}\right)$ by

$$
\left(\pi_{\chi}(f) \xi\right)(g)=\sum_{g_{1} \circ g_{2}=g} f\left(g_{1}\right) \xi\left(g_{2}\right) \quad \text { for all } f \in C_{c}(\mathscr{E}), \xi \in \ell^{2}\left(\mathscr{E}_{\chi}\right) .
$$

In other words, $\pi_{\chi}$ is the left regular representation on $\ell^{2}\left(\mathscr{E}_{\chi}\right)$. Let us define a $\mathrm{C}^{*}$-norm $\|\cdot\|$ on $C_{c}(\mathscr{E})$ by

$$
\|f\|=\sup _{\pi}\|\pi(f)\|,
$$

where $\pi$ runs over all $*$-representations of $C_{c}(\mathscr{E})$. The completion $C^{*}(\mathscr{E})$ of $C_{c}(\mathscr{E})$ under $\|\cdot\|$ is a $C^{*}$-algebra, called the maximal $C^{*}$-algebra of the groupoid $\mathscr{E}$. For more details about groupoid $C^{*}$-algebras, see Renault [31], Khoshkam and Skandalis [24], or Connes [8], Chapter II, §5.

Definition 2.2.2. We define the $\mathrm{C}^{*}$-algebra $C_{k, \infty}$ by letting

$$
C_{k, \infty}=C^{*}(\mathscr{\xi}) \text {. }
$$

By definition, any $*$-representation $\pi$ of $C_{c}(\mathscr{E})$ extends uniquely to a representation of $C_{k, \infty}$, which we still denote $\pi$.

Lemma 2.2.3. For any $*$-automorphism $\sigma$ of $C_{c}(\mathscr{E})$, there exists an unique extension of $\sigma$ to a $*$-automorphism of $C_{k, \infty}$.

Proof. For any $*$-automorphism $\sigma$ of $C_{c}(\mathscr{E})$ and any $*$-representation $\pi$ of $C_{c}(\mathscr{G})$, note that $\pi \circ \sigma$ is a $*$-representation of $C_{c}(\mathscr{E})$. Thus, by definition of the norm $\|\cdot\|, \sigma$ is an isometry: for all $f \in C_{c}(\mathscr{E})$ we have $\|\sigma(f)\|=\|f\|$. The result then follows easily.

For any $g=(\chi, c) \in \mathcal{E}$, put $\mathbf{N} g=\mathbf{N} c$, where $\mathbf{N} c$ is the absolute norm of the fractional ideal $\mathfrak{c}$, defined by $\mathbf{N} c=(\mathbf{N a})^{-1} \mathbf{N} \mathfrak{b}$ for any $\mathfrak{a}, \mathfrak{b} \in \mathfrak{I}_{\mathcal{O}}$ such that $\mathfrak{c}=\mathfrak{a}^{-1} \mathfrak{b}$.

Let us define a one parameter $*$-automorphism group $\left(\sigma_{t}\right)_{t \in \mathbb{R}}$ of $C_{c}(\mathscr{E})$ by

$$
\left(\sigma_{t}(f)\right)(g)=(\mathbf{N} g)^{i t} f(g) \text { for all } t \in \mathbb{R}, f \in C_{c}(\mathcal{E}), g \in \mathcal{E} .
$$

Definition 2.2.4. We still denote $\sigma_{t}$ the unique extension (given by Lemma 2.2.3) of $\sigma_{t}$ to an automorphism of $C_{k, \infty}$. 
It remains to check that the pair $\left(C_{k, \infty},\left(\sigma_{t}\right)\right)$ is a $\mathrm{C}^{*}$-dynamical system in the sense of [4], i.e. that the flow $\left(\sigma_{t}\right)$ is strongly continuous, which means that for any $f \in C_{k, \infty}$, the map $t \mapsto \sigma_{t}(f)$ is continuous.

Lemma 2.2.5. The flow $\left(\sigma_{t}\right)$ on $C_{k, \infty}$ is strongly continuous.

Proof. Let $f \in C_{k, \infty}$. Let us show that the map $t \mapsto \sigma_{t}(f)$ is continuous. Let $\varepsilon>0$. It is enough to show that when $|t|$ is small enough, we have $\left\|f-\sigma_{t}(f)\right\|<\varepsilon$. Let $f^{\prime} \in C_{c}(\mathscr{E})$ be such that $\left\|f-f^{\prime}\right\|<\varepsilon / 3$. Like any $*$-automorphism, $\sigma_{t}$ is an isometry, so we have $\left\|\sigma_{t}(f)-\sigma_{t}\left(f^{\prime}\right)\right\|=\left\|\sigma_{t}\left(f-f^{\prime}\right)\right\|=\left\|f-f^{\prime}\right\|<\varepsilon / 3$, so it is enough to show that when $|t|$ is small enough, we have $\left\|f^{\prime}-\sigma_{t}\left(f^{\prime}\right)\right\|<\varepsilon / 3$. For any $\mathfrak{D} \in \mathfrak{F} \mathcal{O}$, define a function $f_{\mathfrak{d}}^{\prime} \in C_{c}(\mathscr{E})$ by

$$
f_{\mathfrak{D}}^{\prime}(\chi, c)=\left\{\begin{array}{ll}
f^{\prime}(\chi, c) & \text { if } c=\delta, \\
0 & \text { if } c \neq \delta,
\end{array} \quad \text { for all }(\chi, c) \in \mathcal{E}\right.
$$

Note that, as $f^{\prime}$ has compact support, the set $\left\{\mathfrak{D} \in \mathfrak{F} \mathcal{O} \mid f_{\mathfrak{D}}^{\prime} \neq 0\right\}$ is finite, and we have $f^{\prime}=\sum_{\mathfrak{D}} f_{\mathfrak{D}}^{\prime}$. For any $\mathfrak{D}$ we have $\sigma_{t}\left(f_{\mathfrak{D}}^{\prime}\right)=\mathbf{N} \mathbb{d}^{i t} f_{\mathfrak{D}}^{\prime}$, so

$$
\left\|f^{\prime}-\sigma_{t}\left(f^{\prime}\right)\right\| \leqslant \sum_{\mathfrak{D}}\left\|f_{\mathfrak{D}}^{\prime}-\sigma_{t}\left(f_{\mathfrak{D}}^{\prime}\right)\right\| \leqslant \sum_{\mathfrak{D}}\left|1-\mathbf{N} \mathfrak{D}^{i t}\right|\left\|f_{\mathfrak{D}}^{\prime}\right\| .
$$

It is now obvious that when $|t|$ is small enough, this is smaller than $\varepsilon / 3$.

The resulting $\mathrm{C}^{*}$-dynamical system $\left(C_{k, \infty},\left(\sigma_{t}\right)\right)$ is the one that was announced in the introduction of this paper.

\section{Algebraic structure of $\left(C_{k, \infty},\left(\sigma_{t}\right)\right)$}

3.1. The $*$-subalgebra $\mathscr{H}$. In this subsection, we construct a $*$-subalgebra $\mathscr{H}$ which will play the rôle of the algebra $\mathscr{H}$ in the Bost-Connes construction.

For any $a \in \mathfrak{I}_{\mathcal{O}}$, let $\mu_{\mathfrak{a}} \in C_{c}(\mathscr{E})$ be defined by

$$
\mu_{\mathfrak{a}}(\chi, c)=1_{\mathfrak{c}=\mathfrak{a}} \quad \text { for all }(\chi, c) \in \mathscr{G} .
$$

For any $\phi \in H(\operatorname{sgn})$ and for any $\lambda \in \phi\left(\mathbf{C}_{\infty}\right)^{\text {tor }}$, let us define a function $e(\phi, \lambda) \in$ $C_{c}(\mathscr{E})$ by

$$
e(\phi, \lambda)(\chi, c)=1_{c=1} 1_{\chi \in X_{\phi}} \chi(\lambda) \quad \text { for all }(\chi, c) \in \mathscr{E} .
$$

Definition 3.1.1. Let $\mathscr{H}$ denote the $*$-subalgebra of $C_{c}(\mathscr{E})$ generated by the $\mu_{a}$, for all $a \in \mathfrak{I}_{\mathcal{O}}$, and the $e(\phi, \lambda)$, for all $\lambda \in \phi\left(\mathbf{C}_{\infty}\right)^{\text {tor }}$ and for all $\phi \in H(\operatorname{sgn})$. 
We shall later show (Proposition 3.3.5) that $\mathscr{H}$ is dense in $C_{k, \infty}$. For now we concentrate on checking several algebraic relations between the generators $\mu_{\mathfrak{a}}$ and $e(\phi, \lambda)$ (see Proposition 3.1.2). We shall later see (Proposition 3.2.3) that the relations of Proposition 3.1.2 define a presentation of $\mathscr{H}$.

Recall that the inverse map in $\mathcal{E}$ is given by

$$
(\chi, c)^{-1}=\left(\chi^{c}, c^{-1}\right) .
$$

The product law in $C_{c}(\mathscr{E})$, defined by equation (23), can be rewritten as

$$
(f g)(\chi, c)=\sum_{\mathfrak{c}_{2} \in \mathfrak{F}_{\chi}} f\left(\chi^{\mathfrak{c}_{2}}, \mathfrak{c c}_{2}^{-1}\right) g\left(\chi, c_{2}\right) \quad \text { for all } f, g \in C_{c}(\mathscr{G}),(\chi, c) \in \mathscr{E} .
$$

From equation (25), we check that for any $a \in \mathfrak{I}_{\mathcal{O}}$, the adjoint $\mu_{\mathfrak{a}}^{*}$ is given by

$$
\mu_{\mathfrak{a}}^{*}(\chi, c)=1_{\mathfrak{c}=\mathfrak{a}^{-1}} \quad \text { for all }(\chi, c) \in \mathcal{E} .
$$

Using formula (26), we then check that, for all $f \in C_{c}(\mathscr{E})$ and all $(\chi, c) \in \mathscr{E}$, we have

$$
\begin{aligned}
\left(\mu_{\mathfrak{a}} f\right)(\chi, \mathfrak{c}) & =1_{\mathfrak{c a}^{-1} \in \mathfrak{F}_{\chi}} f\left(\chi, \mathfrak{c a}^{-1}\right), \\
\left(f \mu_{\mathfrak{a}}\right)(\chi, \mathfrak{c}) & =f\left(\chi^{\mathfrak{a}}, \mathfrak{c a}^{-1}\right), \\
\left(\mu_{\mathfrak{a}}^{*} f\right)(\chi, \mathfrak{c}) & =f(\chi, \mathfrak{c a}), \\
\left(f \mu_{\mathfrak{a}}^{*}\right)(\chi, \mathfrak{c}) & =1_{\mathfrak{a}^{-1} \in \mathfrak{F}_{\chi}} f\left(\chi^{\left.\mathfrak{a}^{-1}, \mathfrak{c a}\right) .}\right.
\end{aligned}
$$

From that we deduce that $C_{c}(\mathscr{E})$ is unital, with unit $\mu_{1}$ (where, as usual, 1 denotes the principal ideal $(1)=\mathcal{O})$

$$
\mu_{1}=1
$$

and we also deduce the formulas

$$
\begin{aligned}
& \left(\mu_{\mathfrak{a}} f \mu_{\mathfrak{b}}^{*}\right)(\chi, \mathfrak{c})=1_{\mathfrak{c a}^{-1} \in \mathfrak{F}_{\chi}} 1_{\mathfrak{b}^{-1} \in \mathfrak{F}_{\chi}} f\left(\chi^{\mathfrak{b}^{-1}}, \mathfrak{c a}^{-1} \mathfrak{b}\right), \\
& \left(\mu_{\mathfrak{b}}^{*} f \mu_{\mathfrak{a}}\right)(\chi, \mathfrak{c})=f\left(\chi^{\mathfrak{a}}, \mathfrak{c a}^{-1} \mathfrak{b}\right) \text {, } \\
& \left(\mu_{\mathfrak{a}} \mu_{\mathfrak{b}}^{*}\right)(\chi, \mathfrak{c})=1_{\mathfrak{b}^{-1} \in \mathfrak{F}_{\chi}} 1_{\mathfrak{c}=\mathfrak{a} \mathfrak{b}^{-1}}, \\
& \left(\mu_{\mathfrak{b}}^{*} \mu_{\mathfrak{a}}\right)(\chi, c)=1_{\mathfrak{c}=\mathfrak{a} \mathfrak{b}^{-1}} .
\end{aligned}
$$

In particular, for $\mathfrak{b}=\mathfrak{a}$, equation (31) gives

$$
\left(\mu_{\mathfrak{a}} \mu_{\mathfrak{a}}^{*}\right)(\chi, \mathfrak{c})=1_{\mathfrak{a}^{-1} \in \mathfrak{F}_{\chi}} 1_{\mathfrak{c}=1} .
$$

The next proposition establishes some relations between the generators $\mu_{\mathfrak{a}}$ and $e(\phi, \lambda)$. As we said above, it will later turn out that these relations really define a presentation of $\mathscr{H}$ as a $*$-algebra (Proposition 3.2.3) and also a presentation of $C_{k, \infty}$ as a $\mathrm{C}^{*}$-algebra (Proposition 3.3.6). 
Proposition 3.1.2. The functions $\mu_{\mathfrak{a}}$, for $a \in \mathfrak{I}_{\mathcal{O}}$, and $e(\phi, \lambda)$, for $\phi \in H(\mathrm{sgn})$ and $\lambda \in \phi\left(\mathbf{C}_{\infty}\right)^{\text {tor }}$, satisfy the following relations:

$\left(\mathrm{a}_{1}\right) \mu_{\mathfrak{a}}^{*} \mu_{\mathfrak{a}}=\mu_{1}$ for all $a \in \mathfrak{I}_{\mathcal{O}}$.

( $\left.\mathrm{a}_{2}\right) \sum_{\phi} e(\phi, 0)=\mu_{1}$ where $\phi$ runs over $H$ (sgn).

(b) $\mu_{\mathfrak{a}} \mu_{\mathfrak{b}}=\mu_{\mathfrak{a} \mathfrak{b}}$ for all $\mathfrak{a}, \mathfrak{b} \in \mathfrak{I}_{\mathcal{O}}$.

(c) $\mu_{\mathfrak{a}} \mu_{\mathfrak{b}}^{*}=\mu_{\mathfrak{b}}^{*} \mu_{\mathfrak{a}}$ for all $\mathfrak{a}, \mathfrak{b} \in \mathfrak{I}_{\mathcal{O}}$ relatively prime.

$\left(\mathrm{d}_{1}\right) e(\phi, \lambda)^{*}=e(\phi,-\lambda)$ for all $\phi \in H(\mathrm{sgn}), \lambda \in \phi\left(\mathbf{C}_{\infty}\right)^{\text {tor }}$.

$\left(\mathrm{d}_{2}\right) e\left(\phi, \lambda_{1}\right) e\left(\phi, \lambda_{2}\right)=e\left(\phi, \lambda_{1}+\lambda_{2}\right)$ for all $\phi \in H(\operatorname{sgn}), \lambda_{1}, \lambda_{2} \in \phi\left(\mathbf{C}_{\infty}\right)^{\text {tor }}$.

$\left(\mathrm{d}_{3}\right) e\left(\phi^{1}, \lambda_{1}\right) e\left(\phi^{2}, \lambda_{2}\right)=0$ for all $\phi^{1} \neq \phi^{2} \in H(\operatorname{sgn}), \lambda_{i} \in \phi^{i}\left(\mathbf{C}_{\infty}\right)^{\text {tor }}$.

(e) $e(\phi, \lambda) \mu_{\mathfrak{a}}=\mu_{\mathfrak{a}} e\left(a * \phi, \phi_{\mathfrak{a}}(\lambda)\right)$ for all $a \in \mathfrak{I}_{\mathcal{O}}, \phi \in H(\operatorname{sgn}), \lambda \in \phi\left(\mathbf{C}_{\infty}\right)^{\text {tor }}$.

(f) $\mu_{\mathfrak{a}} e(\phi, \lambda) \mu_{\mathfrak{a}}^{*}=\frac{1}{\mathrm{Na}} \sum_{\left(\mathfrak{a}^{-1} * \phi\right)_{\alpha}(\mu)=\lambda} e\left(\mathfrak{a}^{-1} * \phi, \mu\right)$ for all $a \in \mathfrak{I}_{\mathcal{O}}, \phi \in H$ (sgn), $\lambda \in \phi\left(\mathbf{C}_{\infty}\right)^{\text {tor }}$.

Proof. ( $\mathrm{a}_{1}$ ): Equation (32) applied with $\mathfrak{b}=\mathfrak{a}$ gives

$$
\left(\mu_{\mathfrak{a}}^{*} \mu_{\mathfrak{a}}\right)(\chi, \mathfrak{c})=1_{c=1}=\mu_{1}(\chi, c) .
$$

$\left(\mathrm{a}_{2}\right)$ : One checks directly that $\sum_{\phi} e(\phi, 0)=\mu_{1}$.

(b): Equation (27) applied with $f=\mu_{\mathfrak{b}}$ gives

$$
\left(\mu_{\mathfrak{a}} \mu_{\mathfrak{b}}\right)(\chi, \mathfrak{c})=1_{\mathfrak{c a} a^{-1} \in \mathfrak{F}_{\chi}} 1_{\mathfrak{b}=\mathfrak{c} \mathfrak{c}^{-1}}=1_{\mathfrak{b} \in \mathfrak{F}_{\chi}} 1_{\mathfrak{b}=\mathfrak{c a}^{-1}} .
$$

As $\mathfrak{b}$ is in $\mathfrak{I}_{\mathcal{O}}$, we always have $\mathfrak{b} \in \mathfrak{F}_{\chi}$, so we find

$$
\left(\mu_{\mathfrak{a}} \mu_{\mathfrak{b}}\right)(\chi, \mathfrak{c})=1_{\mathfrak{b}=\mathfrak{c a}-1}=1_{\mathfrak{a} \mathfrak{b}=\mathfrak{c}} .
$$

Thus, $\mu_{\mathfrak{a}} \mu_{\mathfrak{b}}=\mu_{\mathfrak{a} \mathfrak{b}}$.

(c): By equations (31), (32) it is enough to show that for all $(\chi, c) \in \mathcal{E}$, we have

$$
1_{\mathfrak{b}^{-1} \in \mathfrak{F}_{\chi}} 1_{\mathfrak{c}=\mathfrak{a} \mathfrak{b}^{-1}}=1_{\mathfrak{c}=\mathfrak{a} \mathfrak{b}^{-1}} .
$$

If $c \neq a \mathfrak{b}^{-1}$, then both sides are zero, so the equality holds. If $c=a \mathfrak{b}^{-1}$, then we have $\mathfrak{a} \mathfrak{b}^{-1} \in \mathfrak{F}_{\chi}$. As $\mathfrak{a}$ and $\mathfrak{b}$ are relatively prime, Lemma 2.1.7 then shows that $\mathfrak{b}^{-1} \in \mathfrak{F}_{\chi}$, so the equality holds.

$\left(\mathrm{d}_{1}\right)$ : For all $\chi \in X$, as $\chi$ is a character we have

$$
\chi(-\lambda)=\overline{\chi(\lambda)} \text { for all } \lambda \in \psi\left(\mathbf{C}_{\infty}\right)^{\text {tor }} .
$$

Relation $\left(\mathrm{d}_{1}\right)$ follows.

$\left(\mathrm{d}_{2}\right)$ and $\left(\mathrm{d}_{3}\right)$ : From equation (26) and the formula $\chi\left(\lambda_{1}+\lambda_{2}\right)=\chi\left(\lambda_{1}\right) \chi\left(\lambda_{2}\right)$, one checks directly that for all $(\chi, c) \in \mathcal{E}$, letting $\psi$ be such that $\chi \in X_{\psi}$, we have

$$
\left(e\left(\phi^{1}, \lambda_{1}\right) e\left(\phi^{2}, \lambda_{2}\right)\right)(\chi, c)=1_{\mathfrak{c}=1} 1_{\phi^{1}=\phi^{2}=\psi} \chi\left(\lambda_{1}+\lambda_{2}\right),
$$


which proves $\left(\mathrm{d}_{2}\right)$ and $\left(\mathrm{d}_{3}\right)$.

(e): By equation (27) and the definition of $e(\phi, \lambda)$, we have, for any $\phi \in H($ sgn $)$, $\lambda \in \phi\left(\mathbf{C}_{\infty}\right)^{\text {tor }}$ and $(\chi, c) \in \mathcal{E}$,

$$
\begin{aligned}
\left(\mu_{\mathfrak{a}} e\left(\mathfrak{a} * \phi, \phi_{\mathfrak{a}}(\lambda)\right)\right)(\chi, c) & =1_{\mathfrak{c a}-1 \in \mathfrak{F} \chi} 1_{\mathfrak{c a}-1=1} 1_{\chi \in X_{\mathfrak{a} * \phi}} \chi\left(\phi_{\mathfrak{a}}(\lambda)\right) \\
& =1_{c a^{-1}=1} 1_{\chi \in X_{\mathfrak{a} * \phi}} \chi^{\mathfrak{a}}(\lambda) .
\end{aligned}
$$

By equation (28), this is equal to $\left(e(\phi, \lambda) \mu_{\mathfrak{a}}\right)(\chi, c)$.

(f): For any $\phi \in H(\operatorname{sgn}), \lambda \in \phi\left(\mathbf{C}_{\infty}\right)^{\text {tor }}$ and $(\chi, c) \in \mathcal{E}$, we have

$$
\begin{aligned}
& \left(\mu_{\mathfrak{a}} e(\phi, \lambda) \mu_{\mathfrak{a}}^{*}\right)(\chi, c)=1_{\mathfrak{c a}^{-1} \in \mathfrak{F}_{\chi}} 1_{\mathfrak{a}^{-1} \in \mathfrak{F}_{\chi}} e(\phi, \lambda)\left(\chi^{\mathfrak{a}^{-1}}, \mathfrak{c}\right) \quad \text { by equation (30) } \\
& =1_{c a^{-1} \in \mathfrak{F}_{\chi}} 1_{a^{-1} \in \mathfrak{F}_{\chi}} 1_{\mathfrak{c}=1} 1_{\chi \in X_{a^{-1} * \phi}} \chi^{\mathfrak{a}^{-1}}(\lambda) \\
& =1_{a^{-1} \in \mathfrak{F}_{\chi}} 1_{c=1} 1_{\chi \in X_{a^{-1} * \phi}} \chi^{a^{-1}}(\lambda) \\
& =1_{a^{-1} \in \mathfrak{F}_{\chi}} 1_{\mathfrak{c}=1} 1_{\chi \in X_{a^{-1} * \phi}}(\mathbf{N a})^{-1} \sum_{\left(a^{-1} * \phi\right)_{a}(\mu)=\lambda} \chi(\mu),
\end{aligned}
$$

where the last equality follows from Lemma 2.1.6. Let us first suppose that $a^{-1} \in \mathfrak{F}_{\chi}$. We then have

$$
\begin{aligned}
\left(\mu_{\mathfrak{a}} e(\phi, \lambda) \mu_{a}^{*}\right)(\chi, c) & =1_{c=1} 1_{\chi \in X_{a}-1_{* \phi}}(\mathbf{N a})^{-1} \sum_{\left(a^{-1} * \phi\right)_{\mathfrak{a}}(\mu)=\lambda} \chi(\mu) \\
& =(\mathbf{N a})^{-1} \sum_{\left(a^{-1} * \phi\right)_{a}(\mu)=\lambda} e\left(a^{-1} * \phi, \mu\right)(\chi, c),
\end{aligned}
$$

so we are done.

Let us now suppose that $a^{-1} \notin \mathfrak{F}_{\chi}$. We then have $1_{\mathfrak{a}^{-1} \in \mathfrak{F} \chi}=0$ and hence $\left(\mu_{\mathfrak{a}} e(\phi, \lambda) \mu_{\mathfrak{a}}^{*}\right)(\chi, c)=0$. Thus, it is enough to show that $\sum_{\left(\mathfrak{a}^{-1} * \phi\right)_{\mathfrak{\alpha}}(\mu)=\lambda} e\left(\mathfrak{a}^{-1} *\right.$ $\phi, \mu)(\chi, c)=0$. We have

$$
\sum_{\left(a^{-1} * \phi\right)_{\mathfrak{a}}(\mu)=\lambda} e\left(a^{-1} * \phi, \mu\right)(\chi, c)=1_{\mathfrak{c}=1} 1_{\chi \in X_{\alpha^{-1} * \phi}} \sum_{\left(a^{-1} * \phi\right)_{\alpha}(\mu)=\lambda} \chi(\mu),
$$

so it is enough to show that if $\chi \in X_{a^{-1} * \phi}$, then $\sum_{\psi_{a}(\mu)=\lambda} \chi(\mu)=0$, where we have set $\psi=a^{-1} * \phi$. Let $\mu_{1} \in \psi\left(\mathbf{C}_{\infty}\right)^{\text {tor }}$ such that $\psi_{a}\left(\mu_{1}\right)=\lambda$ (see Lemma 1.2.6). We then have

$$
\sum_{\psi_{a}(\mu)=\lambda} \chi(\mu)=\sum_{\psi_{\alpha}\left(\mu_{0}\right)=0} \chi\left(\mu_{0}+\mu_{1}\right)
$$

so

$$
\sum_{\psi_{\alpha}(\mu)=\lambda} \chi(\mu)=\left(\sum_{\mu_{0} \in \psi[a]} \chi\left(\mu_{0}\right)\right) \chi\left(\mu_{1}\right)
$$


But by Lemma 2.1.6, since $a^{-1} \notin \mathfrak{F}_{\chi}$, the restriction of $\chi$ to $\psi[a]$ is a non-trivial character of $\psi[a]$, so

$$
\sum_{\mu_{0} \in \psi[a]} \chi\left(\mu_{0}\right)=0
$$

so $\sum_{\psi_{\alpha}(\mu)=\lambda} \chi(\mu)=0$, which completes the proof.

3.2. Presentation of $\mathscr{H}$. The goal of this subsection is to show (Proposition 3.2.3) that the relations (a)-(f) of Proposition 3.1.2 define a presentation of $\mathscr{H}$ as a $*$-algebra.

The proof of the next lemma follows that of Proposition 18 in [3].

Lemma 3.2.1. Let $\tilde{\mathscr{H}}$ be a $*$-algebra with elements $\tilde{\mu}_{\mathfrak{a}}$, for $a \in \mathfrak{I}_{\mathcal{O}}$, and $\tilde{e}(\phi, \lambda)$, for $\phi \in H(\mathrm{sgn})$ and $\lambda \in \phi\left(\mathbf{C}_{\infty}\right)^{\text {tor }}$, satisfying the relations (a)-(f) of Proposition 3.1.2. Let $S$ be the following subset of $\widetilde{\mathscr{H}}$ :

$$
S=\left\{\tilde{\mu}_{\mathfrak{a}} \tilde{e}(\phi, \lambda) \tilde{\mu}_{\mathfrak{b}}^{*} \mid \mathfrak{a}, \mathfrak{b} \in \mathfrak{I}_{\mathcal{O}} \text { relatively prime, } \phi \in H(\operatorname{sgn}), \lambda \in \phi\left(\mathbf{C}_{\infty}\right)^{\mathrm{tor}}\right\} .
$$

Then:

(1) The elements $\tilde{\mu}_{\mathfrak{a}}$, for $a \in \mathfrak{I}_{\mathcal{O}}$, and $\tilde{e}(\phi, \lambda)$, for $\phi \in H(\operatorname{sgn})$ and $\lambda \in \phi\left(\mathbf{C}_{\infty}\right)^{\text {tor }}$, belong to the linear span of $S$. More specifically:

$$
\tilde{\mu}_{a}=\sum_{\phi \in H(\mathrm{sgn})} \tilde{\mu}_{a} \tilde{e}(\phi, 0) \tilde{\mu}_{1}^{*} \text { and } \tilde{e}(\phi, \lambda)=\tilde{\mu}_{1} \tilde{e}(\phi, \lambda) \tilde{\mu}_{1}^{*} .
$$

(2) Let $x_{1}, x_{2} \in S$. For $i=1,2$ write $x_{i}=\mu_{\mathfrak{a}_{i}} e\left(\phi^{i}, \lambda_{i}\right) \mu_{\mathfrak{b}_{i}}^{*}$. Let $\mathfrak{D}=\mathfrak{a}_{2}+\mathfrak{b}_{1}$ be the gcd of $\mathfrak{a}_{2}$ and $\mathfrak{b}_{1}$. Let $c$ be the gcd of $\mathfrak{D}^{-1} \mathfrak{a}_{1} \mathfrak{a}_{2}$ and $\mathfrak{D}^{-1} \mathfrak{b}_{1} \mathfrak{b}_{2}$. Set $\psi=c^{-1} \mathfrak{D}^{-1} \mathfrak{a}_{2} * \phi^{1}$ and $\lambda^{\prime}=\phi_{\mathfrak{D}^{-1} \mathfrak{a}_{2}}^{1}\left(\lambda_{1}\right)+\phi_{\mathfrak{D}^{-1} \mathfrak{b}_{1}}^{2}\left(\lambda_{2}\right)$. Then:

$$
\begin{aligned}
x_{1} x_{2} & =1_{\mathfrak{a}_{2} * \phi^{1}=\mathfrak{b}_{1} * \phi^{2}} \tilde{\mu}_{\mathfrak{D}^{-1} \mathfrak{a}_{1} \mathfrak{a}_{2}} \tilde{e}\left(\mathfrak{D}^{-1} \mathfrak{a}_{2} * \phi^{1}, \lambda^{\prime}\right) \tilde{\mu}_{\mathfrak{D}^{-1} \mathfrak{b}_{1} \mathfrak{b}_{2}}^{*} \\
& =1_{\mathfrak{a}_{2} * \phi^{1}=\mathfrak{b}_{1} * \phi^{2}} \sum_{\psi_{\mathfrak{c}}(\gamma)=\lambda^{\prime}} \tilde{\mu}_{\mathfrak{c}^{-1} \mathfrak{D}^{-1} \mathfrak{a}_{1} \mathfrak{a}_{2}} \tilde{e}(\psi, \gamma) \tilde{\mu}_{\mathfrak{c}^{-1} \mathfrak{D}^{-1} \mathfrak{b}_{1} \mathfrak{b}_{2}}^{*} .
\end{aligned}
$$

In particular, equation (35) shows that $x_{1} x_{2}$ belongs to the $\mathbb{C}$-linear span of $S$.

(3) If the elements $\tilde{\mu}_{\mathfrak{a}}$ and $\tilde{e}(\phi, \lambda)$ generate $\widetilde{\mathscr{H}}$ as a *algebra, then the set $S$ generates $\widetilde{\mathscr{H}}$ as a $\mathbb{C}$-vector space.

Proof. (1) easily follows from relations $\left(\mathrm{a}_{1}\right),\left(\mathrm{a}_{2}\right)$ of Proposition 3.1.2.

(2): We have

$$
x_{1} x_{2}=\mu_{\mathfrak{a}_{1}} e\left(\phi^{1}, \lambda_{1}\right) \mu_{\mathfrak{b}_{1}}^{*} \mu_{\mathfrak{a}_{2}} e\left(\phi^{2}, \lambda_{2}\right) \mu_{\mathfrak{b}_{2}}^{*} .
$$

Using relations $\left(\mathrm{a}_{1}\right),(\mathrm{b})$ and (c) of Proposition 3.1.2, we find

$$
\tilde{\mu}_{\mathfrak{b}_{1}}^{*} \tilde{\mu}_{\mathfrak{a}_{2}}=\tilde{\mu}_{\mathfrak{D}^{-1} \mathfrak{b}_{1}}^{*} \tilde{\mu}_{\searrow}^{*} \tilde{\mu}_{\mathfrak{\delta}} \tilde{\mu}_{\mathfrak{D}^{-1} \mathfrak{a}_{2}}=\tilde{\mu}_{\mathfrak{D}^{-1} \mathfrak{b}_{1}}^{*} \tilde{\mu}_{\mathfrak{D}^{-1} \mathfrak{a}_{2}} .
$$


Hence we get

$$
x_{1} x_{2}=\tilde{\mu}_{\mathfrak{a}_{1}} \tilde{e}\left(\phi^{1}, \lambda_{1}\right) \tilde{\mu}_{\delta^{-1} \mathfrak{a}_{2}} \tilde{\mu}_{\delta^{-1} \mathfrak{b}_{1}}^{*} \tilde{e}\left(\phi^{2}, \lambda_{2}\right) \tilde{\mu}_{\mathfrak{b}_{2}}^{*} .
$$

Using relations (e) and $\left(\mathrm{d}_{1}\right)$ of Proposition 3.1.2, we get

$x_{1} x_{2}=\tilde{\mu}_{\mathfrak{a}_{1}} \tilde{\mu}_{\mathfrak{D}^{-1} \mathfrak{a}_{2}} \tilde{e}\left(\mathfrak{D}^{-1} \mathfrak{a}_{2} * \phi^{1}, \phi_{\mathfrak{D}^{-1} \mathfrak{a}_{2}}^{1}\left(\lambda_{1}\right)\right) \tilde{e}\left(\mathfrak{D}^{-1} \mathfrak{b}_{1} * \phi^{2}, \phi_{\mathfrak{D}^{-1} \mathfrak{b}_{1}}^{2}\left(\lambda_{2}\right)\right) \tilde{\mu}_{\mathfrak{D}}^{* 1} \mathfrak{b}_{1} \tilde{\mu}_{\mathfrak{b}_{2}}^{*}$.

Hence relation (b) of Proposition 3.1.2 gives

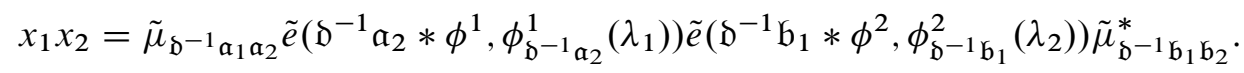

Thus, using relations $\left(\mathrm{d}_{2}\right)$ and $\left(\mathrm{d}_{3}\right)$ of Proposition 3.1.2, we get

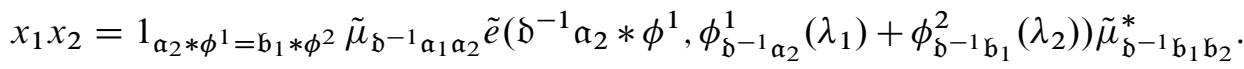

By definition of $\psi, \lambda^{\prime}$ and $c$, and using relation (b) of Proposition 3.1.2, we obtain

$$
x_{1} x_{2}=1_{\mathfrak{a}_{2} * \phi^{1}=\mathfrak{b}_{1} * \phi^{2}} \tilde{\mu}_{\mathfrak{c}^{-1} \mathfrak{D}^{-1} \mathfrak{a}_{1} \mathfrak{a}_{2}}\left(\tilde{\mu}_{\mathfrak{c}} \tilde{e}\left(\mathfrak{c} * \psi, \lambda^{\prime}\right) \tilde{\mu}_{\mathfrak{c}}^{*}\right) \tilde{\mu}_{\mathfrak{c}^{-1} \mathfrak{D}^{-1} \mathfrak{b}_{1} \mathfrak{b}_{2}}^{*} .
$$

Relation (f) of Proposition 3.1.2 then gives the result.

(3): The $\mathbb{C}$-linear span of $S$ contains the generators $\tilde{\mu}_{\mathfrak{a}}$ and $\tilde{e}(\phi, \lambda)$ by (1) and is stable under multiplication by (2). Moreover, it is obviously stable under the involution. Hence it is equal to $\widetilde{\mathscr{H}}$.

Lemma 3.2.2. The functions $\mu_{\mathfrak{a}} e(\phi, \lambda) \mu_{\mathfrak{b}}^{*}$, for $\mathfrak{a}, \mathfrak{b} \in \mathfrak{I}_{\mathcal{O}}$ relatively prime, $\phi \in$ $H(\mathrm{sgn})$ and $\lambda \in \phi\left(\mathbf{C}_{\infty}\right)^{\text {tor }}$, form a basis of $\mathscr{H}$ as a $\mathbb{C}$-vector space.

Proof. By Lemma 3.2.1(3), they generate $\mathscr{H}$ as a $\mathbb{C}$-vector space. Thus we only have to prove that they are linearly independent. Let us suppose that there exist $\alpha_{1}, \ldots, \alpha_{n} \in \mathbb{C}, \mathfrak{a}_{0}, \ldots, \mathfrak{a}_{n}, \mathfrak{b}_{0}, \ldots, \mathfrak{b}_{n} \in \mathfrak{I}_{\mathcal{O}}$ with $\mathfrak{a}_{i}$ relatively prime to $\mathfrak{b}_{i}$ for each $i, \phi^{1}, \ldots, \phi^{n} \in H(\operatorname{sgn})$ and, for each $i, \lambda_{i} \in \phi\left(\mathbf{C}_{\infty}\right)^{\text {tor }}$, such that

$$
\mu_{\mathfrak{a}_{0}} e\left(\phi^{0}, \lambda_{0}\right) \mu_{\mathfrak{b}_{0}}^{*}=\sum_{i=1}^{n} \alpha_{i} \mu_{\mathfrak{a}_{i}} e\left(\phi^{i}, \lambda_{i}\right) \mu_{\mathfrak{b}_{i}}^{*} .
$$

By equation (30) and the definition of $e\left(\phi^{i}, \lambda_{i}\right)$, we have

$$
\begin{aligned}
& \left(\mu_{\mathfrak{a}_{i}} e\left(\phi^{i}, \lambda_{i}\right) \mu_{\mathfrak{b}_{i}}^{*}\right)(\chi, \mathfrak{c}) \\
& \quad=1_{\mathfrak{c a} a_{i}-1 \in \mathfrak{F}_{\chi}} 1_{\mathfrak{b}_{i}-1 \in \mathfrak{F}_{\chi}} 1_{\mathfrak{c a}_{i}{ }^{-1} \mathfrak{b}_{i}=1} 1_{\chi \in X_{\mathfrak{b}_{i}^{-1} * \phi^{i}}} \chi \mathfrak{b}_{i}{ }^{-1}\left(\lambda_{i}\right) \quad \text { for all }(\chi, \mathfrak{c}) \in \mathcal{E} .
\end{aligned}
$$

Thus, the support of $\mu_{\mathfrak{a}_{i}} e\left(\phi^{i}, \lambda_{i}\right) \mu_{\mathfrak{b}_{i}}^{*}$ is included in

$$
\left\{g=(\chi, c) \in \mathcal{E} \mid \chi \in X_{\mathfrak{b}_{i}^{-1} * \phi^{i}} \text { and } \mathrm{c}=a_{i} \mathfrak{b}_{i}{ }^{-1}\right\} \text {. }
$$


Let $I$ denote the set of all $i \neq 0$ such that $a_{i} \mathfrak{b}_{i}{ }^{-1}=a_{0} \mathfrak{b}_{0}{ }^{-1}$ and $\mathfrak{b}_{i}^{-1} * \phi^{i}=\mathfrak{b}_{0}^{-1} * \phi^{0}$. We thus have

$$
\mu_{\mathfrak{a}_{0}} e\left(\phi^{0}, \lambda_{0}\right) \mu_{\mathfrak{b}_{0}}^{*}=\sum_{i \in I} \alpha_{i} \mu_{\mathfrak{a}_{i}} e\left(\phi^{i}, \lambda_{i}\right) \mu_{\mathfrak{b}_{i}}^{*} .
$$

As $a_{i}$ is relatively prime to $\mathfrak{b}_{i}$, we see that for all $i \in I$, we have $a_{i}=a_{0}$ and $\mathfrak{b}_{i}=\mathfrak{b}_{0}$, so $\phi^{i}=\phi^{0}$. Hence we get

$$
\mu_{\mathfrak{a}_{0}} e\left(\phi^{0}, \lambda_{0}\right) \mu_{\mathfrak{b}_{0}}^{*}=\sum_{i \in I} \alpha_{i} \mu_{\mathfrak{a}_{0}} e\left(\phi^{i}, \lambda_{i}\right) \mu_{\mathfrak{b}_{0}}^{*} .
$$

Hence, multiplying by $\mu_{\mathfrak{a}_{0}}^{*}$ on the left and by $\mu_{\mathfrak{b}_{0}}$ on the right, and using relation (a) of Proposition 3.1.2, we get

$$
e\left(\phi^{0}, \lambda_{0}\right)=\sum_{i \in I} \alpha_{i} e\left(\phi^{0}, \lambda_{i}\right)
$$

But the $e\left(\phi^{0}, \lambda\right)$, for $\lambda \in \phi^{0}\left(\mathbf{C}_{\infty}\right)^{\text {tor }}$, are linearly independent (use e.g. the isomorphism $C\left(X_{\phi^{0}} \times\{1\}\right) \simeq C^{*}\left(\phi^{0}\left(\mathbf{C}_{\infty}\right)^{\text {tor }}\right)$ as in Lemma 3.3.2), so this is absurd.

Proposition 3.2.3. The relations (a)-(f) of Proposition 3.1.2 define a presentation of $\mathscr{H}$ as $a *$-algebra.

Proof. Let $\widetilde{\mathscr{H}}$ be another $*$-algebra having elements $\tilde{\mu}_{\mathfrak{a}}$, for $a \in \widetilde{I}_{\mathcal{O}}$, and $\tilde{e}(\phi, \lambda)$, for $\phi \in H(\operatorname{sgn})$ and $\lambda \in \phi\left(\mathbf{C}_{\infty}\right)^{\text {tor }}$, satisfying the relations (a)-(f) of Proposition 3.1.2. We want to show that there exists a unique morphism $\sigma: \mathscr{H} \rightarrow \widetilde{\mathscr{H}}$ such that $\sigma \mu_{\mathfrak{a}}=\tilde{\mu}_{\mathfrak{a}}$ and $\sigma e(\phi, \lambda)=\tilde{e}(\phi, \lambda)$.

The uniqueness is clear by definition of $\mathscr{H}$. Let us now prove existence. By Lemma 3.2.2, we may define a $\mathbb{C}$-linear map $\sigma: \mathscr{H} \rightarrow \widetilde{\mathscr{H}}$ by letting

$$
\sigma\left(\mu_{\mathfrak{a}} e(\phi, \lambda) \mu_{\mathfrak{b}}^{*}\right)=\tilde{\mu}_{\mathfrak{a}} \tilde{e}(\phi, \lambda) \tilde{\mu}_{\mathfrak{b}}^{*}
$$

for all $\mathfrak{a}, \mathfrak{b} \in \mathfrak{I}_{\mathcal{O}}$ relatively prime, $\phi \in H(\operatorname{sgn})$ and $\lambda \in \phi\left(\mathbf{C}_{\infty}\right)^{\text {tor }}$. Clearly, $\sigma\left(f^{*}\right)=$ $\sigma(f)^{*}$. Moreover, Lemma 3.2.1 shows that $\sigma \mu_{a}=\tilde{\mu}_{\mathfrak{a}}, \sigma e(\phi, \lambda)=\tilde{e}(\phi, \lambda)$ and

$$
\sigma\left(f_{1} f_{2}\right)=\sigma\left(f_{1}\right) \sigma\left(f_{2}\right)
$$

which completes the proof.

3.3. Presentation of $\boldsymbol{C}_{\boldsymbol{k}, \infty}$. The goal of this subsection is to show (Proposition 3.3.6) that the relations (a)-(f) of Proposition 3.1.2 define a presentation of $C_{k, \infty}$ as a $\mathrm{C}^{*}$ algebra.

Let $\phi \in H(\mathrm{sgn})$. Let $C_{\phi}$ denote the subset of $C_{c}(\mathcal{E})$ of all functions whose support is a subset of $X_{\phi} \times\{1\}$. 
Lemma 3.3.1. Let $f_{1}, f_{2} \in C_{\phi}$. For all $g \in \mathcal{E}$, we have

$$
\left(f_{1} f_{2}\right)(g)=f_{1}(g) f_{2}(g) \text {. }
$$

Proof. Let $g=(\chi, c) \in \mathcal{E}$. By equation (26), we have

$$
\left(f_{1} f_{2}\right)(\chi, c)=\sum_{\mathfrak{c}_{2} \in \mathfrak{F}_{\chi}} f_{1}\left(\chi^{c_{2}}, \mathfrak{c c}_{2}^{-1}\right) f_{2}\left(\chi, c_{2}\right)
$$

Thus, since $f_{1}, f_{2} \in C_{\phi}$, we can only have a nonzero term when $\mathrm{cc}_{2}^{-1}=1$ and $\mathfrak{c}_{2}=1$. If $\mathfrak{c} \neq 1$ then we get $\left(f_{1} f_{2}\right)(\chi, c)=0$, as expected. If $\mathfrak{c}=1$ we obtain

$$
\left(f_{1} f_{2}\right)(\chi, 1)=f_{1}(\chi, 1) f_{2}(\chi, 1),
$$

as expected.

In particular, we see that for any $f_{1}, f_{2} \in C_{\phi}$, we have $f_{1} f_{2} \in C_{\phi}$. We also have $f_{1}^{*} \in C_{\phi}$. Thus $C_{\phi}$ is a $*$-subalgebra of $C_{c}(\mathcal{G})$.

Let us define a norm $\|\cdot\|_{\phi}$ on $C_{\phi}$ by:

$$
\|f\|_{\phi}=\sup _{g \in \mathscr{E}}|f(g)| \text { for all } f \in C_{\phi} .
$$

Lemma 3.3.2. $C_{\phi}$ is a $C^{*}$-algebra for the norm $\|\cdot\|_{\phi}$. We have isomorphisms of $C^{*}$-algebras

$$
C_{\phi} \simeq C\left(X_{\phi}\right) \simeq C^{*}\left(\phi\left(\mathbf{C}_{\infty}\right)^{\text {tor }}\right)
$$

Proof. The identification $X_{\phi} \times\{1\} \simeq X_{\phi}$ gives a bijection $C_{\phi} \simeq C\left(X_{\phi}\right)$. By Lemma 3.3.1, this is a $*$-isomorphism. By definition of $\|\cdot\|_{\phi}$, this is an isometry, so $\|\cdot\|_{\phi}$ is a $\mathrm{C}^{*}$-norm on $C_{\phi}$. The isomorphism $C\left(X_{\phi}\right) \simeq C^{*}\left(\phi\left(\mathbf{C}_{\infty}\right)^{\text {tor }}\right)$ is a classical result, see Davidson [14], Proposition VII.1.1.

Corollary 3.3.3. $C_{\phi}$ is a $C^{*-s u b a l g e b r a ~ o f ~} C_{k, \infty}$.

Proof. It is a classical result that any injective $*$-morphism between two $\mathrm{C}^{*}$-algebras is an isometry; see [14], Theorem 1.5.5. Apply this to the inclusion map $\iota: C_{\phi} \rightarrow C_{k, \infty}$.

Lemma 3.3.4. The $e(\phi, \lambda)$, for $\lambda \in \phi\left(\mathbf{C}_{\infty}\right)^{\text {tor }}$, generate a norm-dense $*$-subalgebra of $C_{\phi}$.

Proof. By definition of the $e(\phi, \lambda)$, the isomorphism $C_{\phi} \simeq C^{*}\left(\phi\left(\mathbf{C}_{\infty}\right)^{\text {tor }}\right)$ given by Lemma 3.3.2 identifies $e(\phi, \lambda)$ with $\lambda$. But, by definition of $C^{*}\left(\phi\left(\mathbf{C}_{\infty}\right)^{\text {tor }}\right)$, the $\lambda$ generate a dense $*$-subalgebra of $C^{*}\left(\phi\left(\mathbf{C}_{\infty}\right)^{\text {tor }}\right)$, so the result follows. 
Proposition 3.3.5. $\mathcal{H}$ is dense in $C_{k, \infty}$ and any $*$-representation of $\mathscr{H}$ extends uniquely to a representation of $C_{k, \infty}$.

Proof. Let us first prove density. Since $C_{c}(\mathscr{G})$ is dense in $C_{k, \infty}$, it is enough to show that any $f \in C_{c}(\mathscr{E})$ can be approached by elements of $\mathscr{H}$. Let $f \in C_{c}(\mathscr{E})$. As $f$ has compact support, there is a finite subset $\left\{c_{1}, \ldots, c_{n}\right\} \subset \mathfrak{F}_{\mathcal{O}}$ such that for all $(\chi, c) \in \mathscr{G}$, if $c \notin\left\{c_{1}, \ldots, c_{n}\right\}$, then $f(\chi, c)=0$. Let $f_{i}$ be defined by

$$
f_{i}(\chi, c)=1_{\mathfrak{c}=c_{i}} f(\chi, c) \quad \text { for all }(\chi, c) \in \mathscr{E} .
$$

We have

$$
f=f_{1}+\cdots+f_{n} .
$$

It is thus enough to show that each of the $f_{i}$ can be approached by elements of $\mathscr{H}$. Let $i \in \mathbb{N}$ such that $1 \leqslant i \leqslant n$. Write $\mathfrak{c}_{i}=\mathfrak{a}_{i}^{-1} \mathfrak{b}_{i}$, with $\mathfrak{a}_{i}, \mathfrak{b}_{i} \in \mathfrak{I}_{\mathcal{O}}$ relatively prime. Let $f_{i}^{\prime}=\mu_{\mathfrak{a}_{i}} f_{i} \mu_{\mathfrak{b}_{i}}^{*}$. We have $f_{i}=\mu_{\mathfrak{a}_{i}}^{*} f_{i}^{\prime} \mu_{\mathfrak{b}_{i}}$, so it is enough to show that each of the $f_{i}^{\prime}$ can be approached by elements of $\mathscr{H}$. By equation (30), we have, for all $(\chi, c) \in \mathcal{E}$,

$$
f_{i}^{\prime}(\chi, \mathfrak{c})=1_{\mathfrak{c a}_{i}^{-1} \in \mathfrak{F}_{\chi}} 1_{\mathfrak{b}_{i}^{-1} \in \mathfrak{F}_{\chi}} f_{i}\left(\chi^{\mathfrak{b}_{i}^{-1}}, \mathfrak{c c}_{i}\right) .
$$

Thus, the support of $f_{i}^{\prime}$ is a subset of $X \times\{1\}$. For $\phi \in H(\operatorname{sgn})$, let $f_{i, \phi}^{\prime}$ be defined by

$$
f_{i, \phi}^{\prime}(\chi, c)=1_{\chi \in X_{\phi}} f_{i}^{\prime}(\chi, c) \quad \text { for all }(\chi, c) \in \mathcal{E} .
$$

We have

$$
f_{i}^{\prime}=\sum_{\phi \in H(\mathrm{sgn})} f_{i, \phi}^{\prime},
$$

so it is enough to show that each of the $f_{i, \phi}^{\prime}$ can be approached by elements of $\mathscr{H}$. We have $f_{i, \phi}^{\prime} \in C_{\phi}$, so the result follows from Lemma 3.3.4.

Now let us prove that any $*$-representation of $\mathscr{H}$ extends uniquely to a representation of $C_{k, \infty}$. Uniqueness follows from the density of $\mathscr{H}$ in $C_{k, \infty}$. Let us show existence. Let $\pi$ be a $*$-representation of $\mathscr{H}$. By definition of $C_{k, \infty}$, it is enough to show that $\pi$ extends to a $*$-representation of $C_{c}(\mathscr{E})$. The construction we just made with the $f_{i}, f_{i}^{\prime}$ and $f_{i, \phi}^{\prime}$ shows that as a $*$-algebra, $C_{c}(\mathscr{G})$ is generated by the $C_{\phi}$, for $\phi \in H(\operatorname{sgn})$, and the $\mu_{\mathfrak{a}}$, for $a \in \mathfrak{I}_{\mathcal{O}}$. It is thus enough to show that the restriction of $\pi$ to the group algebra $\mathbb{C}\left[\phi\left(\mathbf{C}_{\infty}\right)^{\text {tor }}\right]$ extends to a representation of $C_{\phi}$. But this follows from Lemma 3.3.2

Proposition 3.3.6. The relations (a)-(f) of Proposition 3.1.2 define a presentation of $C_{k, \infty}$ as a $C^{*}$-algebra.

Proof. Let $\widetilde{C}$ be another $C^{*}$-algebra having elements $\tilde{\mu}_{\mathfrak{a}}$, for $\mathfrak{a} \in \mathfrak{I}_{\mathcal{O}}$, and $\tilde{e}(\phi, \lambda)$, for $\phi \in H$ (sgn) and $\lambda \in \phi\left(\mathbf{C}_{\infty}\right)^{\text {tor }}$, satisfying the relations (a)-(f) of Proposition 3.1.2. 
We want to show that there exists a unique morphism $\sigma: C_{k, \infty} \rightarrow \widetilde{C}$ such that $\sigma \mu_{\mathfrak{a}}=\tilde{\mu}_{\mathfrak{a}}$ and $\sigma e(\phi, \lambda)=\tilde{e}(\phi, \lambda)$.

Uniqueness follows from the density of $\mathscr{H}$ in $C_{k, \infty}$, see Proposition 3.3.5. Let us prove existence.

Let $\widetilde{\mathscr{H}}$ denote the $*$-algebra generated by the $\tilde{\mu}_{\mathfrak{a}}$ and the $\tilde{e}(\phi, \lambda)$. By the universal property of $\mathscr{H}$ (Proposition 3.2.3), there exists a $*$-morphism $\sigma: \mathscr{H} \rightarrow \widetilde{\mathscr{H}}$ such that $\sigma \mu_{\mathfrak{a}}=\tilde{\mu}_{\mathfrak{a}}$ and $\sigma e(\phi, \lambda)=\tilde{e}(\phi, \lambda)$. Composing it with the inclusion $\widetilde{\mathscr{H}} \rightarrow \widetilde{C}$ gives a $*$-representation of $\mathscr{H}$. By Proposition 3.3.5, this representation extends to a *-morphism from $C_{k, \infty}$ into $\widetilde{C}$, so we are done.

The flow $\left(\sigma_{t}\right)$ has a simple expression for this presentation: one checks directly that

$$
\sigma_{t}\left(\mu_{\mathfrak{a}}\right)=\mathbf{N} a^{i t} \mu_{\mathfrak{a}} \quad \text { for all } t \in \mathbb{R}, a \in \mathfrak{s}_{\mathcal{O}}
$$

and

$$
\sigma_{t} e(\phi, \lambda)=e(\phi, \lambda) \quad \text { for all } t \in \mathbb{R}, \phi \in H(\operatorname{sgn}), \lambda \in \phi\left(\mathbf{C}_{\infty}\right)^{\text {tor }}
$$

3.4. Galois symmetry of $\left(C_{\boldsymbol{k}, \infty},\left(\sigma_{\boldsymbol{t}}\right)\right)$. Recall that an action of $\operatorname{Gal}(K / k)$ on $X$ has been defined by equation (19).

Let $\operatorname{Gal}(K / k)$ act by $*$-automorphisms on $C_{c}(\mathcal{G})$ by

$$
(\sigma f)(\chi, c)=f(\sigma \chi, c) \quad \text { for all } \sigma \in \operatorname{Gal}(K / k), f \in C_{c}(\mathscr{G}),(\chi, c) \in \mathcal{E}
$$

Definition 3.4.1. We still denote $(\sigma, f) \mapsto \sigma f$ the unique extension (given by Lemma 2.2.3) of this action to an action of $\operatorname{Gal}(K / k)$ on $C_{k, \infty}$.

One checks directly that the action of $\mathrm{Gal}(K / k)$ on the generators is given by

$$
\sigma \mu_{\mathfrak{a}}=\mu_{\mathfrak{a}} \quad \text { for all } \sigma \in \operatorname{Gal}(K / k), a \in \mathfrak{I}_{\mathcal{O}}
$$

and

$$
\sigma(e(\phi, \lambda))=e(\sigma \phi, \sigma \lambda) \text { for all } \sigma \in \operatorname{Gal}(K / k), \phi \in H(\operatorname{sgn}), \lambda \in \phi\left(\mathbf{C}_{\infty}\right)^{\text {tor }} .
$$

Proposition 3.4.2. The group $\mathrm{Gal}(K / k)$, endowed with its profinite topology, is a topological symmetry group of $\left(C_{k, \infty},\left(\sigma_{t}\right)\right)$. In other words, the action of $\operatorname{Gal}(K / k)$ on $C_{k, \infty}$ is faithful, continuous, and commutes with the flow $\left(\sigma_{t}\right)$, i.e.,

$$
\sigma\left(\sigma_{t}(f)\right)=\sigma_{t}(\sigma f) \quad \text { for all } \sigma \in \operatorname{Gal}(K / k), t \in \mathbb{R}, f \in C_{k, \infty}
$$

Proof. By Lemma 2.2.3, it is enough to check equation (40) for $f \in C_{c}(\mathcal{E})$, which is easily done by going back to the definitions.

Let us check that the action of $\operatorname{Gal}(K / k)$ on $C_{k, \infty}$ is faithful. Let $\sigma \in \operatorname{Gal}(K / k)$ with $\sigma \neq 1$. Let $\phi \in H(\operatorname{sgn})$. If $\sigma \phi \neq \phi$ then it is clear that $\sigma$ acts non-trivially 
on $C_{k, \infty}$. If $\sigma \phi=\phi$ then, by definition of $H^{+}$, we have $\sigma \in \operatorname{Gal}\left(K / H^{+}\right)$. By definition of $K$, the action of $\operatorname{Gal}\left(K / H^{+}\right)$on $\phi\left(\mathbf{C}_{\infty}\right)^{\text {tor }}$ is faithful. Thus there exists $\lambda \in \phi\left(\mathbf{C}_{\infty}\right)^{\text {tor }}$ such that $\sigma \lambda \neq \lambda$, so $e(\phi, \sigma \lambda) \neq e(\phi, \lambda)$. Thus, by equation (39), $\sigma e(\phi, \lambda) \neq e(\phi, \lambda)$, so the action of $\operatorname{Gal}(K / k)$ on $C_{k, \infty}$ is faithful.

Let us check that the action of $\operatorname{Gal}(K / k)$ on $C_{k, \infty}$ is continuous. Let $f \in C_{k, \infty}$ and $\varepsilon>0$. By Proposition 3.3.5, the subalgebra $\mathscr{H}$ is dense in $C_{k, \infty}$, so there exists $f_{0} \in \mathscr{H}$ with $\left\|f-f_{0}\right\|<\varepsilon / 3$. Write $f_{0}$ in the basis provided by Lemma 3.2.2,

$$
f_{0}=\sum_{i \in I} c_{i} \mu_{\mathfrak{a}_{i}} e\left(\phi^{i}, \lambda_{i}\right) \mu_{\mathfrak{b}_{i}}^{*},
$$

where $I$ is a finite set and where, for all $i \in I$, we have $c_{i} \in \mathbb{C}, \mathfrak{a}_{i}, \mathfrak{b}_{i} \in \mathfrak{I}_{\mathcal{O}}$ relatively prime, $\phi^{i} \in H(\mathrm{sgn})$, and $\lambda_{i} \in \phi^{i}\left(\mathbf{C}_{\infty}\right)^{\text {tor }}$. Let $K_{0}$ be the extension of $k$ generated by the $\lambda_{i}$ and all their conjugates under $\operatorname{Gal}(K / k)$. Thus, $K_{0} / k$ is a finite Galois subextension of $K / k$. Let $V=\operatorname{Gal}\left(K / K_{0}\right)$. By definition of the profinite topology, $V$ is a neighborhood of $1 \mathrm{in} \operatorname{Gal}(K / k)$. For all $\sigma \in V$, we have $\sigma f_{0}=f_{0}$. We have $\left\|\sigma f-f_{0}\right\|=\left\|\sigma\left(f-f_{0}\right)\right\|=\left\|f-f_{0}\right\|<\varepsilon / 3$, so we find $\|\sigma f-f\|<2 \varepsilon / 3$. Let $W$ denote the open ball of radius $\varepsilon / 3$ centered at $f$. For all $f^{\prime} \in W$, we have $\left\|\sigma f^{\prime}-\sigma f\right\|=\left\|\sigma\left(f^{\prime}-f\right)\right\|=\left\|f^{\prime}-f\right\|<\varepsilon / 3$, whence $\left\|\sigma f^{\prime}-f\right\|<\varepsilon$, which completes the proof of the continuity.

3.5. The Galois-fixed subalgebra. In this subsection, we introduce two $\mathrm{C}^{*}$-subalgebras of $C_{k, \infty}$, and it will turn out (Lemma 3.5.2) that they are the same one.

The first one, denoted by $C^{*}\left(\widetilde{I}_{\mathcal{O}}\right)$, is the $\mathrm{C}^{*}$-subalgebra of $C_{k, \infty}$ generated by the $\mu_{\mathfrak{a}}$, for all $a \in \mathfrak{I}_{\mathcal{O}}$. The second one, denoted by $C_{k, \infty}^{\mathrm{Gal}(K / k)}$, is the subset of $C_{k, \infty}$ of all fixed points under the action of $\operatorname{Gal}(K / k)$. This is a $\mathrm{C}^{*}$-subalgebra of $C_{k, \infty}$.

Let

$$
\Phi: \mathfrak{I}_{\mathcal{O}} \rightarrow \mathbb{N}
$$

and

$$
\mathbf{M}: \mathfrak{I}_{\mathcal{O}} \rightarrow \mathbb{Z}
$$

denote the Euler totient and Möbius inversion functions respectively, i.e., $\Phi$ and $\mathbf{M}$ are the multiplicative functions defined, for all primes $p$ and for all $n \geqslant 0$, by

$$
\Phi\left(\mathfrak{p}^{n}\right)=\mathbf{N} \mathfrak{p}^{n}-1_{n \geqslant 1} \mathbf{N} \mathfrak{p}^{n-1}
$$

and

$$
\mathbf{M}\left(\mathfrak{p}^{n}\right)=1_{n=0}-1_{n=1} .
$$

Note that we have, for all $a \in \mathfrak{I}_{\mathcal{O}}$,

$$
\Phi(\mathfrak{a})=\sum_{\mathfrak{b} \mid \mathfrak{a}} \mathbf{M}\left(\mathfrak{b}^{-1} \mathfrak{a}\right) \mathbf{N} \mathfrak{b} .
$$


Lemma 3.5.1. For all $\phi \in H(\mathrm{sgn})$, for all $a \in \mathfrak{I}_{\mathcal{O}}$, the $\mathcal{O}$-module $\phi[\mathfrak{a}]$ has exactly $\Phi(\mathfrak{a})$ generators.

Proof. Let $a=\prod_{i} p_{i}^{n_{i}}$ be the factorization of $a$. Since the $\mathfrak{p}_{i}$ are relatively prime, we have $a=\bigcap_{i} p_{i}^{n_{i}}$, so by equations (15) and (16), we have

$$
\phi[\mathfrak{a}]=\bigoplus_{i} \phi\left[\mathfrak{p}_{i}^{n_{i}}\right],
$$

so it is enough to do the proof when $a$ is a prime power, which is then easy.

The proof of the next lemma has been inspired by that of Proposition 21 (b) in [3] and of Proposition 4.1 (3) in [17].

Lemma 3.5.2. The two subalgebras $C^{*}\left(\mathfrak{I}_{\mathcal{O}}\right)$ and $C_{k, \infty}^{\mathrm{Gal}(K / k)}$ of $C_{k, \infty}$ are the same:

$$
C^{*}\left(\mathfrak{\Im}_{\mathcal{O}}\right)=C_{k, \infty}^{\mathrm{Gal}(K / k)}
$$

Definition 3.5.3. We let $C_{1}$ denote this $\mathrm{C}^{*}$-algebra:

$$
C_{1}=C^{*}\left(\mathfrak{I}_{\mathcal{O}}\right)=C_{k, \infty}^{\mathrm{Gal}(K / k)} .
$$

This notation will be justified in Subsection 4.4, where $C_{1}$ will be viewed as a spectral subspace of $C_{k, \infty}$ for the action of $\operatorname{Gal}(K / k)$.

Proof. One inclusion is clear: $C_{k, \infty}^{\mathrm{Gal}(K / k)}$ contains $C^{*}\left(\mathfrak{I}_{\mathcal{O}}\right)$. Let us check the other inclusion. The Galois group $\operatorname{Gal}(K / k)$ is endowed with its profinite topology, so it is a compact abelian group. Let $d \sigma$ be the normalized Haar measure on it. Let us consider the map $\mathbf{E}$ defined by

$$
\begin{aligned}
\mathbf{E}: C_{k, \infty} & \rightarrow C_{k, \infty}^{\mathrm{Gal}(K / k),} \\
x & \mapsto \int_{\mathrm{Gal}(K / k)} \sigma(x) d \sigma .
\end{aligned}
$$

By Proposition 3.3.5, $\mathscr{H}$ is dense in $C_{k, \infty}$, so $\mathbf{E}(\mathcal{H})$ is dense in $C_{k, \infty}^{\mathrm{Gal}(K / k)}$. But, by Lemma 3.2.1, $\mathscr{H}$ is the linear span of the $\mu_{\mathfrak{a}} e(\phi, \lambda) \mu_{\mathfrak{b}}^{*}$, for $\mathfrak{a}, \mathfrak{b} \in \mathfrak{\Im}_{\mathcal{O}}, \phi \in H(\operatorname{sgn})$, and $\lambda \in \phi\left(\mathbf{C}_{\infty}\right)^{\text {tor }}$. Thus $\mathbf{E}(\mathscr{H})$ is the linear span of the $\mu_{\mathfrak{a}} \mathbf{E}(e(\phi, \lambda)) \mu_{\mathfrak{b}}^{*}$. Hence, it is enough to show that for all $\phi \in H(\operatorname{sgn})$ and for all $\lambda \in \phi\left(\mathbf{C}_{\infty}\right)^{\text {tor }}$, the element $\mathbf{E}(e(\phi, \lambda))$ belongs to $C^{*}\left(\widetilde{\Im}_{\mathcal{O}}\right)$.

So let $\phi \in H(\operatorname{sgn})$ and $\lambda \in \phi\left(\mathbf{C}_{\infty}\right)^{\text {tor }}$. Let us first assume that $\lambda=0$. The group $\operatorname{Gal}\left(H^{+} / k\right)$ acts transitively on $H(\operatorname{sgn})$ (see Theorem 1.3.9). By Galois theory, 
the restriction map $\operatorname{Gal}(K / k) \rightarrow \operatorname{Gal}\left(H^{+} / k\right)$ is surjective. Hence $\operatorname{Gal}(K / k)$ acts transitively on $H(\operatorname{sgn})$. Thus, by relation $\left(\mathrm{a}_{2}\right)$ in Proposition 3.1.2, we get

$$
\mathbf{E}(e(\phi, \lambda))=1 / h(\operatorname{sgn}),
$$

where $h(\operatorname{sgn})$ is the cardinal of $H(\operatorname{sgn})$. So the proof is complete.

Let us now assume that $\lambda \neq 0$. Let

$$
a=\operatorname{ann}_{\mathcal{O}}(\lambda)=\left\{a \in \mathcal{O} \mid \phi_{a}(\lambda)=0\right\} .
$$

We have $\lambda \in \phi[\mathfrak{a}]$ and, for all $\mathfrak{b} \neq \mathfrak{a}$ such that $\mathfrak{b} \mid \mathfrak{a}, \lambda \notin \phi[\mathfrak{b}]$. So $\lambda$ is a generator of the $\mathcal{O}$-module $\phi[a]$. Let $K_{a}$ denote the extension of $H^{+}$generated by the elements of $\phi[a]$. By [20], Theorem 16.2, $\operatorname{Gal}\left(K_{\mathfrak{a}} / k\right)$ acts transitively on the set $\mathfrak{X}_{\mathfrak{a}}$ defined by

$$
\mathfrak{X}_{a}=\{(\psi, \mu) \mid \psi \in H(\operatorname{sgn}), \mu \text { is a generator of } \psi[a]\} .
$$

By Galois theory, the map $\operatorname{Gal}(K / k) \rightarrow \operatorname{Gal}\left(K_{a} / k\right)$ is surjective, so $\operatorname{Gal}(K / k)$ also acts transitively on $\mathfrak{X}_{a}$. Thus $\mathbf{E}(e(\phi, \lambda))$ only depends on $a$. We therefore note

$$
\mathbf{E}\left(e\left(a^{-1}\right)\right)=\mathbf{E}(e(\phi, \lambda)) .
$$

Relation (f) of Proposition 3.1.2 gives, for all $\psi \in H(\operatorname{sgn})$ and $\mathfrak{b} \in \mathfrak{I}_{\mathcal{O}}$,

$$
\mu_{\mathfrak{b}} e(\psi, 0) \mu_{\mathfrak{b}}^{*}=\frac{1}{\mathbf{N b}} \sum_{\mu \in\left(\mathfrak{b}^{-1} * \psi\right)[\mathfrak{b}]} e\left(\mathfrak{b}^{-1} * \psi, \mu\right) .
$$

Thus equation $\left(\mathrm{a}_{2}\right)$ of Proposition 3.1.2 gives

$$
\mu_{\mathfrak{b}} \mu_{\mathfrak{b}}^{*}=\frac{1}{\mathbf{N} \mathfrak{b}} \sum_{\psi \in H(\operatorname{sgn})} \sum_{\mu \in\left(\mathfrak{b}^{-1} * \psi\right)[\mathfrak{b}]} e\left(\mathfrak{b}^{-1} * \psi, \mu\right) .
$$

Applying $\mathbf{E}$ to this equality and using Lemma 3.5.1, we get

$$
\mathbf{N b} \mu_{\mathfrak{b}} \mu_{\mathfrak{b}}^{*}=h(\operatorname{sgn}) \sum_{\mathfrak{c} \mid \mathfrak{b}} \Phi(\mathfrak{c}) \mathbf{E}\left(e\left(\mathrm{c}^{-1}\right)\right),
$$

where $h(\operatorname{sgn})$ is the cardinal of $H(\operatorname{sgn})$. Doing a Möbius inversion, we then find

$$
h(\operatorname{sgn}) \Phi(\mathfrak{b}) \mathbf{E}\left(e\left(\mathfrak{b}^{-1}\right)\right)=\sum_{\mathfrak{c} \mid \mathfrak{b}} \mathbf{M}\left(\mathfrak{c}^{-1} \mathfrak{b}\right) \mathbf{N} c \mu_{\mathfrak{c}} \mu_{\mathfrak{c}}^{*}
$$

Thus, for all $\mathfrak{b} \in \mathfrak{I}_{\mathcal{O}}$, we get the following explicit expression of $\mathbf{E}\left(e\left(\mathfrak{b}^{-1}\right)\right)$ as an element of $C^{*}\left(\mathfrak{I}_{\mathcal{O}}\right)$ :

$$
\mathbf{E}\left(e\left(\mathfrak{b}^{-1}\right)\right)=\frac{\sum_{\mathfrak{c} \mid \mathfrak{b}} \mathbf{M}\left(\mathfrak{c}^{-1} \mathfrak{b}\right) \mathbf{N} c \mu_{\mathfrak{c}} \mu_{\mathfrak{c}}^{*}}{h(\operatorname{sgn}) \Phi(\mathfrak{b})}=\frac{\sum_{\mathfrak{c} \mid \mathfrak{b}} \mathbf{M}\left(\mathfrak{c}^{-1} \mathfrak{b}\right) \mathbf{N} c \mu_{\mathfrak{c}} \mu_{\mathfrak{c}}^{*}}{h(\operatorname{sgn}) \sum_{\mathfrak{c} \mid \mathfrak{b}} \mathbf{M}\left(c^{-1} \mathfrak{b}\right) \mathbf{N} c}
$$


Proposition 3.5.4. $C_{1}$ is isomorphic to the universal $C^{*}$-algebra generated by elements $\tilde{\mu}_{\mathfrak{a}}$, for $\mathfrak{a} \in \mathfrak{I}_{\mathcal{O}}$, subject to the relations $\left(\mathrm{a}_{1}\right)$, (b) and (c) of Proposition 3.1.2.

Proof. This follows directly from Proposition 3.3.6 and Lemma 3.5.2.

3.6. Admissible characters. Some ideas in this subsection have been inspired by [17], §5. Our main goal here is to prove Proposition 3.6.9, which will be useful for the classification of extremal $\mathrm{KMS}_{\beta}$ states at low temperature.

Lemma 3.6.1. Let $\chi \in X$. Let $\phi \in H(\mathrm{sgn})$ be such that $\chi \in X_{\phi}$. The following conditions are equivalent:

(1) For any maximal ideal $\mathfrak{p} \in \mathfrak{I}_{\mathcal{O}}$, the restriction of $\chi$ to $\phi[\mathfrak{p}]$ is non-trivial.

(2) For any $\mathfrak{b} \in \mathfrak{I}_{\mathcal{O}}$ different from 1 , the restriction of $\chi$ to $\phi[\mathfrak{b}]$ is non-trivial.

(3) $\mathfrak{F}_{\chi}=\mathfrak{s}_{\mathcal{O}}$

Proof. (2) $\Rightarrow$ (1) is trivial. (1) $\Rightarrow(2)$ : Since $\mathfrak{b} \neq 1$ there exists a maximal ideal $\mathfrak{p}$ dividing $\mathfrak{b}$. By equation (14), we then have $\phi[\mathfrak{p}] \subset \phi[\mathfrak{b}]$, so the result follows. (2) $\Rightarrow$ (3): Let $c \in \mathfrak{F}_{\chi}$. Write $\mathfrak{c}=\mathfrak{b}^{-1} \mathfrak{a}$ with $\mathfrak{a}, \mathfrak{b} \in \mathfrak{I}_{\mathcal{O}}$ relatively prime. By Lemma 2.1.7, we have $\mathfrak{b}^{-1} \in \mathfrak{F}_{\chi}$. Thus, by Lemma 2.1.6, the restriction of $\chi$ to $\phi[\mathfrak{b}]$ is trivial, so $\mathfrak{b}=1$, so $\mathfrak{c} \in \widetilde{\mathfrak{I}}_{\mathcal{O}}$. (3) $\Rightarrow(2)$ : Let $\mathfrak{b} \in \mathfrak{I}_{\mathcal{O}}$ with $\mathfrak{b} \neq 1$. We have $\mathfrak{b}^{-1} \notin \mathfrak{F}_{\chi}$, so the result follows by Lemma 2.1.6.

Definition 3.6.2. A character $\chi \in X$ is said to be admissible if it satisfies the above equivalent conditions. Let $X^{\text {adm }}$ denote the topological subspace of $X$ of admissible elements.

Recall that $A_{f}$ is the ring of finite adèles of $k$ with respect to $\mathcal{O}$. Thus, $A_{f}$ is the restricted product of the $k_{\mathfrak{p}}$ with respect to the $\mathcal{O}_{\mathfrak{p}}$, where $\mathfrak{p}$ runs over all finite places of $k$.

The following lemma is well known.

Lemma 3.6.3. Let $a \in \mathfrak{I}_{\mathcal{O}}$. The diagonal map $\iota: k \hookrightarrow A_{f}$ induces an $\mathcal{O}$-module isomorphism

$$
k / \mathfrak{a} \stackrel{\sim}{\longrightarrow} \bigoplus_{\mathfrak{p}} k_{\mathfrak{p}} / \mathfrak{a}_{\mathfrak{p}},
$$

where $\mathfrak{p}$ runs over all finite places of $k, k_{\mathfrak{p}}$ is the completion of $k$ at $\mathfrak{p}$, and $\mathfrak{a}_{\mathfrak{p}}$ is the closure of $a$ in $k_{\mathfrak{p}}$.

Proof. Let $R=\prod_{\mathfrak{p}} \mathfrak{a}_{\mathfrak{p}} \subset A_{f}$. This contains $\iota(\mathfrak{a})$. Hence $\iota$ induces a map

$$
k / \mathfrak{a} \rightarrow A_{f} / R .
$$


This map is an $\mathcal{O}$-module morphism. It is injective because $\iota^{-1}(R)=a$. By the strong approximation theorem (Theorem 1.1.1), the range of $\iota$ is dense in $A_{f}$. But by definition of the restricted product, $R$ is an open subset of $A_{f}$. Hence $\iota$ induces a surjection modulo $R$. Thus $\iota$ induces an isomorphism of $\mathcal{O}$-modules $k / \mathfrak{a} \simeq A_{f} / R$. But $A_{f} / R=\bigoplus_{\mathfrak{p}} k_{\mathfrak{p}} / \mathfrak{a}_{\mathfrak{p}}$, so the result follows.

Lemma 3.6.4. For any ideal $a \in \mathfrak{I}_{\mathcal{O}}$ and for any finite place $\mathfrak{p}$ of $k$, there exists a character $\chi$ of $k_{\mathfrak{p}} / \mathfrak{a}_{\mathfrak{p}}$ whose restriction to $\mathfrak{p}^{-1} \mathfrak{a}_{\mathfrak{p}} / \mathfrak{a}_{\mathfrak{p}}$ is non-trivial.

Proof. Let $\mathbb{F}_{\mathfrak{p}}$ denote the residue field of $\mathcal{O}_{\mathfrak{p}}$. This is a finite extension of $\mathbb{F}_{p}$. The ring $\mathcal{O}_{\mathfrak{p}}$ is principal (as is any local ring of a Dedekind ring), so its maximal ideal $\mathfrak{p} \mathcal{O}_{\mathfrak{p}}$ is equal to $u \mathcal{O}_{\mathfrak{p}}$ for some $u \in \mathcal{O}_{\mathfrak{p}}$. Now $\mathfrak{a}_{\mathfrak{p}}$ is also an ideal of $\mathcal{O}_{\mathfrak{p}}$, so it is equal to $u^{v} \mathcal{O}_{\mathfrak{p}}$ for some $v \geqslant 0$. Hence we have $\mathfrak{p}^{-1} \mathfrak{a}_{\mathfrak{p}} / \mathfrak{a}_{\mathfrak{p}}=u^{v-1} \mathcal{O}_{\mathfrak{p}} / u^{v} \mathcal{O}_{\mathfrak{p}}$. But we have $k_{\mathfrak{p}}=\mathbb{F}_{\mathfrak{p}}((u))$ and $\mathcal{O}_{\mathfrak{p}}=\mathbb{F}_{\mathfrak{p}}[[u]]$, so we can define a character $\chi$ on $k_{\mathfrak{p}} / \mathfrak{a}_{\mathfrak{p}}$ by letting

$$
\chi\left(\sum_{k \in \mathbb{Z}} a_{k} u^{k}\right)=\exp \left(2 i \pi \operatorname{Tr}_{\mathbb{F}_{p}}^{\mathbb{F}_{\mathfrak{p}}}\left(a_{v-1}\right) / p\right) .
$$

The restriction of $\chi$ to $\mathfrak{p}^{-1} \mathfrak{a}_{\mathfrak{p}} / \mathfrak{a}_{\mathfrak{p}}$ is non-trivial since we have $\chi\left(u^{v-1}\right)=\exp (2 i \pi / p)$.

Lemma 3.6.5. For any ideal $a \in \mathfrak{I}_{\mathcal{O}}$, there exists a character $\chi$ of $k / a$ whose restriction to $\mathfrak{p}^{-1} \mathfrak{a}_{\mathfrak{p}} / \mathfrak{a}_{\mathfrak{p}}$, for any finite place $\mathfrak{p}$ of $k$, is non-trivial.

Proof. Use Lemma 3.6.3 to identify $k / \mathfrak{a}$ with $\bigoplus_{\mathfrak{p}} k_{\mathfrak{p}} / \mathfrak{a}_{\mathfrak{p}}$. For all $\mathfrak{p}$, let $\chi_{\mathfrak{p}}$ be a character of $k_{\mathfrak{p}} / \mathfrak{a}_{\mathfrak{p}}$ as given by the preceding lemma. Let $\chi=\prod_{\mathfrak{p}} \chi_{\mathfrak{p}}$. Then $\chi$ is a character of $k / a$ which has the required property.

Lemma 3.6.6. For any $\phi \in H(\mathrm{sgn})$, there exists an admissible character $\chi \in X_{\phi}$. In particular, $X^{\mathrm{adm}}$ is non-empty.

Proof. Let $L$ denote the lattice corresponding to $\phi$. Write $L=\xi$ a with $\xi \in \mathbf{C}_{\infty}^{*}$ and $a \in \mathfrak{I}_{\mathcal{O}}$. Let $\chi_{0}$ be a character of $k / a$ as given by Lemma 3.6.5. Define a character $\chi$ of $\phi\left(\mathbf{C}_{\infty}\right)^{\text {tor }}$ by

$$
\chi(\lambda)=\chi_{0}\left(e_{L}^{-1}(\lambda) / \xi\right)
$$

Then $\chi$ is admissible.

Lemma 3.6.7. For any $\chi \in X^{\mathrm{adm}}$, the map $\mathfrak{I}_{\mathcal{O}} \rightarrow X, a \mapsto \chi^{\mathfrak{a}}$, is injective.

Proof. By definition of admissibility and equation (21), we have $\mathfrak{F}_{\chi^{\alpha}}=\mathfrak{a}^{-1} \mathfrak{I}_{\mathcal{O}}$, so the result follows.

Lemma 3.6.8. For any $\chi \in X^{\mathrm{adm}}$ and for any $\sigma \in \mathrm{Gal}(K / k)$, we have $\sigma \chi \in X^{\mathrm{adm}}$. 
Proof. The actions of $\operatorname{Gal}(K / k)$ and of $\mathfrak{\Im}_{\mathcal{O}}$ on $X$ commute with one another. Hence, $\mathfrak{F}_{\sigma \chi}=\mathfrak{F}_{\chi}=\mathfrak{\Im}_{\mathcal{O}}$. Hence $\sigma \chi$ is admissible.

Proposition 3.6.9. For any $\chi \in X^{\mathrm{adm}}$, the map $\operatorname{Gal}(K / k) \rightarrow X^{\mathrm{adm}}, \sigma \mapsto \sigma \chi$, is injective.

Proof. Let $\phi \in H$ (sgn) such that $\chi \in X_{\phi}$. Let $1 \neq \sigma \in \operatorname{Gal}(K / k)$. Suppose that $\sigma \chi=\chi$. We have $\sigma \chi \in X_{\sigma^{-1} \phi}$, so $\sigma^{-1} \phi=\phi$. Thus $\sigma \phi=\phi$, so by definition of $H^{+}$, we see that $\sigma \in \operatorname{Gal}\left(K / H^{+}\right)$. Also $\sigma$ induces a map

$$
\sigma: \phi\left(\mathbf{C}_{\infty}\right)^{\text {tor }} \rightarrow \phi\left(\mathbf{C}_{\infty}\right)^{\text {tor }}
$$

For any $\lambda \in \phi\left(\mathbf{C}_{\infty}\right)^{\text {tor }}$, for any $a \in \mathcal{O}$, we have $\phi_{a}(\sigma \lambda)=\left(\sigma \phi_{a}\right)(\sigma \lambda)=\sigma\left(\phi_{a}(\lambda)\right)$, so $\sigma$ is an $\mathcal{O}$-module automorphism of $\phi\left(\mathbf{C}_{\infty}\right)^{\text {tor }}$. Let $L$ denote the lattice corresponding to $\phi$. Write $L=\xi$ a with $\xi \in \mathbf{C}_{\infty}^{*}$ and $a \in \mathfrak{I}_{\mathcal{O}}$. Thus, we have $\mathcal{O}$-module isomorphisms

$$
k / \mathfrak{a} \stackrel{\xi}{\rightarrow} k L / L \stackrel{e_{L}}{\rightarrow} \phi\left(\mathbf{C}_{\infty}\right)^{\text {tor }},
$$

which we use to identify $k / \mathfrak{a}$ with $\phi\left(\mathbf{C}_{\infty}\right)^{\text {tor }}$ as $\mathcal{O}$-modules. Thus, $\sigma$ is seen as an $\mathcal{O}$-module automorphism of $k / \mathfrak{a}$. Use Lemma 3.6 .3 to identify $k / \mathfrak{a}$ with $\bigoplus_{\mathfrak{p}} k_{\mathfrak{p}} / \mathfrak{a}_{\mathfrak{p}}$. For any finite place $\mathfrak{p}$ of $k$, writing $k_{\mathfrak{p}}$ as a field of Laurent series as in the proof of Lemma 3.6.4, one sees that $k_{\mathfrak{p}} / \mathfrak{a}_{\mathfrak{p}} \simeq k_{\mathfrak{p}} / \mathcal{O}_{\mathfrak{p}}$ as $\mathcal{O}_{\mathfrak{p}}$-modules, hence as $\mathcal{O}$-modules. Hence $\operatorname{End}_{\mathcal{O}}\left(k_{\mathfrak{p}} / \mathfrak{a}_{\mathfrak{p}}\right)=\mathcal{O}_{\mathfrak{p}}$, acting by multiplication. Thus

$$
\operatorname{End}_{\mathcal{O}}(k / \mathfrak{a})=\prod_{\mathfrak{p}} \mathcal{O}_{\mathfrak{p}}
$$

View $\sigma$ as an element of $\operatorname{End}_{\mathcal{O}}(k / \mathfrak{a})$ and write $\sigma=\prod_{\mathfrak{p}} \sigma_{\mathfrak{p}}$ with $\sigma_{\mathfrak{p}} \in \mathcal{O}_{\mathfrak{p}}$ for all $\mathfrak{p}$.

By definition of $K$, the action of $\operatorname{Gal}\left(K / H^{+}\right)$on $\phi\left(\mathbf{C}_{\infty}\right)^{\text {tor }}$ is faithful. Thus, as an $\mathcal{O}$-module automorphism of $\phi\left(\mathbf{C}_{\infty}\right)^{\text {tor }}$, we have $\sigma \neq 1$. Thus, there exists a $\mathfrak{p}$ such that $\sigma_{\mathfrak{p}} \neq 1$, so $\sigma_{\mathfrak{p}}-1 \in \mathcal{O}_{\mathfrak{p}}-\{0\}$. Since $\chi$ is admissible, there exists $\lambda \in \phi[\mathfrak{p}]$ such that $\chi(\lambda) \neq 1$. View $\lambda$ as an element of $\mathfrak{p}^{-1} \mathfrak{a}_{\mathfrak{p}} / \mathfrak{a}_{\mathfrak{p}}$. Let $\tilde{\lambda} \in \mathfrak{p}^{-1} \mathfrak{a}_{\mathfrak{p}} \subset k_{\mathfrak{p}}$ be a representative of $\lambda$. Let $\tilde{\mu}=\left(\sigma_{\mathfrak{p}}-1\right)^{-1} \tilde{\lambda} \in k_{\mathfrak{p}}$. Let $\mu$ denote the class of $\tilde{\mu}$ in $k_{\mathfrak{p}} / \mathfrak{a}_{\mathfrak{p}}$. We have $\left(\sigma_{\mathfrak{p}}-1\right) \mu=\lambda$, so $(\sigma-1) \mu=\lambda$, so

$$
\chi((\sigma-1) \mu) \neq 1
$$

and so

$$
\chi(\sigma \mu) \neq \chi(\mu)
$$

which is absurd since $\sigma \chi=\chi$. 
3.7. Irreducibility of regular representations at admissible characters. The goal of this subsection is to show that the regular representations of $\mathcal{E}$ associated to admissible characters are irreducible. This will be used to classify extremal KMS states at low temperature.

Recall that for any $\chi \in X$ we defined the regular representation $\pi_{\chi}$ of $C_{c}(\mathscr{E})$ by equation (24). By definition of $C_{k, \infty}, \pi_{\chi}$ extends uniquely to a representation of $C_{k, \infty}$.

Recall that $X^{\text {adm }}$ is the subset of $X$ of admissible elements.

Lemma 3.7.1. For all $\chi \in X^{\mathrm{adm}}$, the regular representation $\pi_{\chi}$ of $C_{k, \infty}$ is irreducible.

Proof. Let $\chi \in X^{\mathrm{adm}}$. Let $\phi$ be such that $\chi \in X_{\phi}$. The representation $\pi_{\chi}$ is a map $C_{k, \infty} \rightarrow B \ell^{2}\left(\mathscr{E}_{\chi}\right)$. Identify $\mathfrak{F}_{\chi}$ with $\mathscr{E}_{\chi}$ through the map $\mathrm{c} \mapsto(\chi, \mathrm{c})$. As $\chi$ is admissible, we have $\mathfrak{F}_{\chi}=\mathfrak{I}_{\mathcal{O}}$. Thus $\mathscr{E}_{\chi}$ is identified with $\mathfrak{I}_{\mathcal{O}}$. Let $A \in B \ell^{2}\left(\mathfrak{I}_{\mathcal{O}}\right)$ such that

$$
\pi_{\chi}(f) A=A \pi_{\chi}(f) \text { for all } f \in C_{k, \infty} .
$$

Let us show that $A$ is a scalar multiple of the identity. For that let us first prove that $A$ is diagonal. Let $\left(\varepsilon_{\mathfrak{c}}\right)_{\mathfrak{c} \in \mathfrak{I}_{\mathcal{O}}}$ be the standard orthonormal basis of $\ell^{2}\left(\mathfrak{I}_{\mathcal{O}}\right)$ : in other words, for all $\mathfrak{c}, a \in \mathfrak{I}_{\mathcal{O}}, \varepsilon_{\mathfrak{c}}(\mathfrak{a})=1_{\mathfrak{a}=\mathfrak{c}}$. Let $\left(a_{\mathfrak{c}, \mathfrak{b}}\right)$ be the matrix representing $A$ in this basis. Thus we have

$$
A \varepsilon_{\mathfrak{D}}=\sum_{\mathfrak{c}} a_{\mathfrak{c}, \mathfrak{D}} \varepsilon_{\mathfrak{c}} \quad \text { for all } \mathrm{c} \in \mathfrak{I}_{\mathcal{O}}
$$

Using equation (24), we check that

$$
\pi_{\chi}\left(\mu_{\mathfrak{a}}\right) \varepsilon_{\mathfrak{b}}=\varepsilon_{\mathfrak{a} \mathfrak{b}} \quad \text { for all } \mathfrak{a} \in \mathfrak{s}_{\mathcal{O}}, \mathfrak{b} \in \mathfrak{s}_{\mathcal{O}}
$$

and

$\pi_{\chi}(e(\psi, \lambda)) \varepsilon_{\mathfrak{b}}=1_{\psi=\mathfrak{b}^{-1} * \phi} \chi^{\mathfrak{b}}(\lambda) \varepsilon_{\mathfrak{b}}$ for all $\psi \in H(\operatorname{sgn}), \lambda \in \psi\left(\mathbf{C}_{\infty}\right)^{\text {tor }}, \mathfrak{b} \in \widetilde{I}_{\mathcal{O}}$.

Now let $\underline{\lambda}=\left(\lambda_{\psi}\right)_{\psi \in H(\operatorname{sgn})}$ be a family with $\lambda_{\psi} \in \psi\left(\mathbf{C}_{\infty}\right)^{\text {tor }}$ for all $\psi \in H(\operatorname{sgn})$. Let

$$
e(\underline{\lambda})=\sum_{\psi \in H(\mathrm{sgn})} e\left(\psi, \lambda_{\psi}\right) .
$$

We have

$$
\pi_{\chi}(e(\underline{\lambda})) \varepsilon_{\mathfrak{b}}=\chi^{\mathfrak{b}}\left(\lambda_{\mathfrak{b}^{-1} * \phi}\right) \varepsilon_{\mathfrak{b}} \quad \text { for all } \mathfrak{b} \in \mathfrak{I}_{\mathcal{O}} .
$$

Thus, for all $\mathfrak{b} \in \mathfrak{s}_{\mathcal{O}}$, we get

$$
\begin{aligned}
& A \pi_{\chi}(e(\underline{\lambda})) \varepsilon_{\mathfrak{b}}=\sum_{\mathfrak{a} \in \mathfrak{I}_{\mathcal{O}}} a_{\mathfrak{a}, \mathfrak{b}} \chi^{\mathfrak{b}}\left(\lambda_{\mathfrak{b}-1 * \phi}\right) \varepsilon_{\mathfrak{a}}, \\
& \pi_{\chi}(e(\underline{\lambda})) A \varepsilon_{\mathfrak{b}}=\sum_{\mathfrak{a} \in \mathfrak{I}_{\mathcal{O}}} a_{\mathfrak{a}, \mathfrak{b}} \chi^{\mathfrak{a}}\left(\lambda_{\mathfrak{a}^{-1} * \phi}\right) \varepsilon_{\mathfrak{a}} .
\end{aligned}
$$


Thus, for all $\mathfrak{a}, \mathfrak{b} \in \mathfrak{I}_{\mathcal{O}}$ with $a_{\mathfrak{a}, \mathfrak{b}} \neq 0$ and for all $\underline{\lambda}$, we get

$$
\chi^{\mathfrak{b}}\left(\lambda_{\mathfrak{b}^{-1} * \phi}\right)=\chi^{\mathfrak{a}}\left(\lambda_{a^{-1} * \phi}\right) .
$$

If $\mathfrak{b}^{-1} * \phi \neq a^{-1} * \phi$, since $\chi$ is admissible, we can obviously choose $\underline{\lambda}$ to make equation (46) fail. Thus we have $\mathfrak{b}^{-1} * \phi=\mathfrak{a}^{-1} * \phi$. By letting $\underline{\lambda}$ vary, we see that $\chi^{\mathfrak{b}}$ and $\chi^{\mathfrak{a}}$ are the same character of $\left(\mathfrak{a}^{-1} * \phi\right)\left(\mathbf{C}_{\infty}\right)^{\text {tor }}$. Thus $\chi^{\overline{\mathfrak{a}}}=\chi^{\mathfrak{b}}$. Thus, as $\chi$ is admissible, by Lemma 3.6.7, we find $\mathfrak{a}=\mathfrak{b}$. Thus $\left(a_{\mathfrak{c}, \mathfrak{\delta}}\right)$ is a diagonal matrix. Finally, using the equality $A \pi_{\chi}\left(\mu_{a}\right)=\pi_{\chi}\left(\mu_{a}\right) A$ for all $a \in \mathfrak{s}_{\mathcal{O}}$, one sees that the diagonal entries $\left(a_{\mathfrak{c}, \mathfrak{c}}\right)$ are all equal, so that $\left(a_{\mathfrak{c}, \mathfrak{b}}\right)$ is a scalar multiple of the identity matrix. Thus $\pi_{\chi}$ is an irreducible representation.

3.8. A lemma on the action of $\operatorname{Gal}(K / \boldsymbol{k})$ on $\mathscr{H}$. In this subsection we prove an important lemma which we shall use in Subsections 4.4 and 4.5 .

Definition 3.8.1. Let $F$ be a set of finite places of $k$. An ideal $c \in \mathfrak{I}_{\mathcal{O}}$ is said to be $F$-localized if all its prime divisors belong to $F$.

Definition 3.8.2. Let $\searrow \in \mathfrak{S}_{\mathcal{O}}$. Let $F_{\searrow}$ be the set of all places of $k$ dividing $\delta$. We define $\mathscr{H}[§]$ to be the $*$-algebra generated by the $\mu_{\mathfrak{a}}$, for all $F_{\mathfrak{D}}$-localized ideals $a \in \mathfrak{I}_{\mathcal{O}}$, and the $e(\phi, \lambda)$, for all $\phi \in H(\operatorname{sgn})$ and $\lambda \in \phi[\delta]$.

Note that for any $\searrow \in \mathfrak{I}_{\mathcal{O}}, \operatorname{Gal}\left(K / K_{\mathfrak{D}}\right)$ acts trivially on $\mathscr{H}[§]$. Thus the action of $\operatorname{Gal}(K / k)$ on $\mathscr{H}[§]$ gives an action of the quotient group $\operatorname{Gal}\left(K_{\triangleright} / k\right)=$ $\operatorname{Gal}(K / k) / \mathrm{Gal}\left(K / K_{\searrow}\right)$ on $\mathscr{H}[\S]$ (remember that the field $K_{\searrow}$ was defined in Definition 1.3.10).

Lemma 3.8.3. Let $\mathfrak{D} \in \mathfrak{I}_{\mathcal{O}}$. Let $\mathfrak{p}$ be a maximal ideal of $\mathfrak{I}_{\mathcal{O}}$ not dividing $\mathfrak{D}$. Let $\sigma_{\mathfrak{p}}=\left(\mathfrak{p}, K_{\mathfrak{D}} / k\right) \in \operatorname{Gal}\left(K_{\mathfrak{D}} / k\right)$ be the Artin automorphism of $K_{\mathfrak{D}}$ associated to $\mathfrak{p}$. For all $x \in \mathscr{H}[\delta]$, we have

$$
x \mu_{\mathfrak{p}}=\mu_{\mathfrak{p}} \sigma_{\mathfrak{p}}(x) .
$$

Proof. Let $A$ denote the subset of $\mathscr{H}[\delta]$ of all elements $x$ such that equation (47) holds. Obviously, $A$ is a $\mathbb{C}$-subalgebra of $\mathscr{H}[\delta]$. But $\mathscr{H}[\delta]$ is generated as a $\mathbb{C}$ algebra by the $\mu_{\mathfrak{a}}$, the $\mu_{\mathfrak{a}}^{*}$ and the $e(\phi, \lambda)$, for all $F_{\mathfrak{D}}$-localized ideals a $\in \mathfrak{I}_{\mathcal{O}}$, all $\phi \in H(\mathrm{sgn})$ and all $\lambda \in \phi[\delta]$. Indeed, by relation $\left(\mathrm{d}_{1}\right)$ of Proposition 3.1.2, we have $e(\phi, \lambda)^{*}=e(\phi,-\lambda)$. Hence in order to prove that $A=\mathscr{H}[\delta]$, it is enough to check that $\mu_{\mathfrak{a}} \in A, \mu_{\mathfrak{a}}^{*} \in A$ and $e(\phi, \lambda) \in A$ for any $F_{\mathfrak{D}}$-localized ideal $a \in \mathfrak{I}_{\mathcal{O}}$, any $\phi \in H(\operatorname{sgn})$ and any $\lambda \in \phi[\delta]$.

Let $a \in \mathfrak{I}_{\mathcal{O}}$ be a $F_{\mathfrak{D}}$-localized ideal. By relation (b) of Proposition 3.1.2, we have $\mu_{\mathfrak{a}} \mu_{\mathfrak{p}}=\mu_{\mathfrak{p}} \mu_{\mathfrak{a}}=\mu_{\mathfrak{p}} \sigma_{\mathfrak{p}}\left(\mu_{\mathfrak{a}}\right)$, so $\mu_{\mathfrak{a}} \in A$. As $a$ is $F_{\mathfrak{D}}$-localized and $\mathfrak{p}$ does not divide $\mathfrak{b}$, relation (c) of Proposition 3.1.2 gives $\mu_{\mathfrak{a}}^{*} \mu_{\mathfrak{p}}=\mu_{\mathfrak{p}} \mu_{\mathfrak{a}}^{*}=\mu_{\mathfrak{p}} \sigma_{\mathfrak{p}}\left(\mu_{\mathfrak{a}}^{*}\right)$, so $\mu_{a}^{*} \in A$. 
Now let $\phi \in H$ (sgn) and $\lambda \in \phi[\delta]$. We have

$$
\begin{aligned}
e(\phi, \lambda) \mu_{\mathfrak{p}} & =\mu_{\mathfrak{p}} e\left(\mathfrak{p} * \phi, \phi_{\mathfrak{p}}(\lambda)\right) & & \text { by relation (e) of Proposition 3.1.2 } \\
& =\mu_{\mathfrak{p}} e\left(\sigma_{\mathfrak{p}} \phi, \phi_{\mathfrak{p}}(\lambda)\right) & & \text { by Theorem 1.3.9 } \\
& =\mu_{\mathfrak{p}} e\left(\sigma_{\mathfrak{p}} \phi, \sigma_{\mathfrak{p}}(\lambda)\right) & & \text { by Theorem 1.3.11, as } \mathfrak{p} \nmid \mathfrak{D} \\
& =\mu_{\mathfrak{p}} \sigma_{\mathfrak{p}}(e(\phi, \lambda)) . & &
\end{aligned}
$$

Thus $e(\phi, \lambda) \in A$, which completes the proof.

\section{4. $\mathrm{KMS}_{\beta}$ equilibrium states of $\left(C_{k, \infty},\left(\sigma_{t}\right)\right)$}

4.1. The Galois-invariant $\mathrm{KMS}_{\boldsymbol{\beta}}$ state at any temperature. The goal of this subsection is to construct (Proposition 4.1.2), for any $\beta \in \mathbb{R}_{+}^{*}$, a Galois-invariant $\mathrm{KMS}_{\beta}$ state $\varphi_{\beta}$ of $\left(C_{k, \infty},\left(\sigma_{t}\right)\right)$. We shall also show (Proposition 4.1.3) that $\varphi_{\beta}$ is the only Galois-invariant $\mathrm{KMS}_{\beta}$ state of $\left(C_{k, \infty},\left(\sigma_{t}\right)\right)$.

Proposition 3.5.4 shows that $C_{1}$ is isomorphic to the infinite tensor product

$$
C_{1}=\bigotimes_{\mathfrak{p}} \tau_{\mathfrak{p}}
$$

where $\mathfrak{p}$ runs over the finite places of $k$ and where, for each $\mathfrak{p}, \tau_{\mathfrak{p}}$ is the (Toeplitz) $\mathrm{C}^{*}$-algebra generated by $\mu_{\mathfrak{p}}$. Note that the $\tau_{\mathfrak{p}}$ are nuclear.

Let $\beta \in \mathbb{R}_{+}^{*}$. For each $\mathfrak{p}$, define a state $\varphi_{\beta, \mathfrak{p}}$ on $\tau_{\mathfrak{p}}$ by

$$
\varphi_{\beta, \mathfrak{p}}\left(\mu_{\mathfrak{p}}^{n} \mu_{\mathfrak{p}}^{* m}\right)=1_{n=m} \mathbf{N p}^{-n \beta} \quad \text { for all } n, m \geqslant 0 .
$$

Define a state $\varphi_{\beta}$ on $C_{1}$ by

$$
\varphi_{\beta}=\bigotimes_{\mathfrak{p}} \varphi_{\beta, \mathfrak{p}}
$$

Note that we have

$$
\varphi_{\beta}\left(\mu_{\mathfrak{a}} \mu_{\mathfrak{b}}^{*}\right)=1_{\mathfrak{a}=\mathfrak{b}} \mathbf{N} \mathfrak{a}^{-\beta} \quad \text { for all } \mathfrak{a}, \mathfrak{b} \in \mathfrak{I}_{\mathcal{O}} .
$$

Recall that the map E: $C_{k, \infty} \rightarrow C_{1}$ was defined in equation (41).

Definition 4.1.1. We extend $\varphi_{\beta}$ to a state on $C_{k, \infty}$ by letting

$$
\varphi_{\beta}(f)=\varphi_{\beta}(\mathbf{E}(f)) \quad \text { for all } f \in C_{k, \infty} .
$$

Proposition 4.1.2. For any $\beta \in \mathbb{R}_{+}^{*}$, the state $\varphi_{\beta}$ on $C_{k, \infty}$ is a $K M S_{\beta}$ state of $\left(C_{k, \infty},\left(\sigma_{t}\right)\right)$. In particular, the state $\varphi_{\beta}$ on $C_{1}$ is a $K M S_{\beta}$ state of $\left(C_{1},\left(\sigma_{t}\right)\right)$. 
Proof. For any $f_{1}, f_{2} \in C_{k, \infty}$, we look for a bounded holomorphic function $F_{\beta, f_{1}, f_{2}}$ on the strip $0<\operatorname{Im} z<\beta$ realizing the $\mathrm{KMS}_{\beta}$ property for the state $\varphi_{\beta}$ and the pair $\left(f_{1}, f_{2}\right)$.

Since $\mathcal{H}$ is a dense $\left(\sigma_{t}\right)$-invariant $*$-subalgebra of $C_{k, \infty}$, by [4], $\$ 5.3 .1$, it is enough to do that for $f_{1}, f_{2} \in \mathcal{H}$. In Lemma 3.2.2, we found a basis of $\mathscr{H}$ as a $\mathbb{C}$-vector space. Obviously, it is enough to check the $\mathrm{KMS}_{\beta}$ condition in the case when $f_{1}$ and $f_{2}$ are elements of that basis. Thus, write $f_{1}=\mu_{\mathfrak{a}_{1}} e\left(\psi^{1}, \lambda_{1}\right) \mu_{\mathfrak{b}_{1}}^{*}$ and $f_{2}=\mu_{\mathfrak{a}_{2}} e\left(\psi^{2}, \lambda_{2}\right) \mu_{\mathfrak{b}_{2}}^{*}$ with $\mathfrak{a}_{i}, \mathfrak{b}_{i} \in \mathfrak{s}_{\mathcal{O}}$ relatively prime, with $\psi^{i} \in H(\mathrm{sgn})$ and with $\lambda_{i} \in \psi^{i}\left(\mathbf{C}_{\infty}\right)^{\text {tor }}$. By Lemma 3.2.1(2),

$$
f_{1} f_{2}=1_{\mathfrak{a}_{2} * \psi^{1}=\mathfrak{b}_{1} * \psi^{2}} \mu_{\delta^{-1} \mathfrak{a}_{1} \mathfrak{a}_{2}} e\left(\mathfrak{\delta}^{-1} \mathfrak{a}_{2} * \psi^{1}, \lambda^{\prime}\right) \mu_{\mathfrak{D}^{-1} \mathfrak{b}_{1} \mathfrak{b}_{2}}^{*}
$$

where $\mathfrak{D}$ is the $\operatorname{gcd}$ of $\mathfrak{a}_{2}$ and $\mathfrak{b}_{1}$ and $\lambda^{\prime}=\psi_{\mathfrak{D}^{-1} \mathfrak{a}_{2}}^{1}\left(\lambda_{1}\right)+\psi_{\mathfrak{D}^{-1} \mathfrak{b}_{1}}^{2}\left(\lambda_{2}\right)$. We thus have

$$
\mathbf{E}\left(f_{1} f_{2}\right)=1_{\mathfrak{a}_{2} * \psi^{1}=\mathfrak{b}_{1} * \psi^{2}} \mu_{\mathfrak{D}^{-1} \mathfrak{a}_{1} \mathfrak{a}_{2}} \mathbf{E}\left(e\left(\mathfrak{D}^{-1} \mathfrak{a}_{2} * \psi^{1}, \lambda^{\prime}\right)\right) \mu_{\mathfrak{D}^{-1} \mathfrak{b}_{1} \mathfrak{b}_{2}}^{*}
$$

Let $c=\operatorname{ann}_{\mathcal{O}}\left(\lambda^{\prime}\right)$. Using equation (43), we deduce

$$
\mathbf{E}\left(f_{1} f_{2}\right)=1_{\mathfrak{a}_{2} * \psi^{1}=\mathfrak{b}_{1} * \psi^{2}} \mu_{\mathfrak{D}^{-1} \mathfrak{a}_{1} \mathfrak{a}_{2}} \frac{\sum_{\mathfrak{f} \mid \mathfrak{c}} \mathbf{M}\left(\mathfrak{f}^{-1} \mathfrak{c}\right) \mathbf{N} \mathfrak{f} \mu_{\mathfrak{f}} \mu_{\mathfrak{f}}^{*}}{h(\operatorname{sgn}) \Phi(\mathfrak{c})} \mu_{\mathfrak{D}^{-1} \mathfrak{b}_{1} \mathfrak{b}_{2}}^{*},
$$

where $h(\operatorname{sgn})$ is the cardinal of $H(\operatorname{sgn})$. Using the formula for $\varphi_{\beta}\left(\mu_{\mathfrak{a}} \mu_{\mathfrak{b}}^{*}\right)$ given in equation (48), we then get

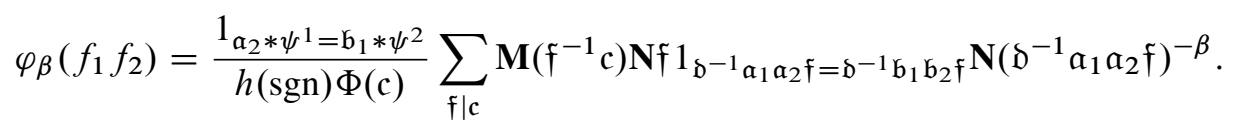

Now the condition $\mathfrak{D}^{-1} \mathfrak{a}_{1} \mathfrak{a}_{2} \mathfrak{f}=\mathfrak{D}^{-1} \mathfrak{b}_{1} \mathfrak{b}_{2} \mathfrak{f}$ is equivalent to $\mathfrak{a}_{1} \mathfrak{a}_{2}=\mathfrak{b}_{1} \mathfrak{b}_{2}$ and, as $\mathfrak{a}_{i}$ is relatively prime to $\mathfrak{b}_{i}$, this is equivalent to $\mathfrak{a}_{1}=\mathfrak{b}_{2}$ and $\mathfrak{a}_{2}=\mathfrak{b}_{1}$. We thus get

$$
\varphi_{\beta}\left(f_{1} f_{2}\right)=\frac{1_{\mathfrak{a}_{1}=\mathfrak{b}_{2}} 1_{\mathfrak{a}_{2}=\mathfrak{b}_{1}} 1_{\psi^{1}=\psi^{2}}}{h(\operatorname{sgn}) \Phi(\mathfrak{c})} \sum_{\mathfrak{f} \mid \mathfrak{c}} \mathbf{M}\left(\mathfrak{f}^{-1} \mathfrak{c}\right) \mathbf{N} \mathfrak{f} \mathbf{N}\left(\mathfrak{D}^{-1} \mathfrak{a}_{1} \mathfrak{a}_{2} \mathfrak{f}\right)^{-\beta} .
$$

Now if $\mathfrak{a}_{2}=\mathfrak{b}_{1}$, then $\mathfrak{b}=\mathfrak{a}_{2}=\mathfrak{b}_{1}$, and so $\mathfrak{c}=\operatorname{ann}_{\mathcal{O}}\left(\lambda_{1}+\lambda_{2}\right)$. Summing this up, we have

$$
\varphi_{\beta}\left(f_{1} f_{2}\right)=\frac{1_{\mathfrak{a}_{1}=\mathfrak{b}_{2}} 1_{\mathfrak{a}_{2}=\mathfrak{b}_{1}} 1_{\psi^{1}=\psi^{2}}}{h(\operatorname{sgn}) \Phi(\mathfrak{c})} \sum_{\mathfrak{f} \mid \mathfrak{c}} \mathbf{M}\left(\mathfrak{f}^{-1} \mathfrak{c}\right) \mathbf{N} \mathfrak{f} \mathbf{N}\left(\mathfrak{a}_{1} \mathfrak{f}\right)^{-\beta}
$$

where $c=\operatorname{ann}_{\mathcal{O}}\left(\lambda_{1}+\lambda_{2}\right)$. Swapping $f_{1}$ with $f_{2}$ amounts to swapping 1 with 2 in the indices, so we get

$$
\varphi_{\beta}\left(f_{2} f_{1}\right)=\frac{1_{\mathfrak{a}_{1}=\mathfrak{b}_{2}} 1_{\mathfrak{a}_{2}=\mathfrak{b}_{1}} 1_{\psi^{1}=\psi^{2}}}{h(\operatorname{sgn}) \Phi(\mathfrak{c})} \sum_{\mathfrak{f} \mid \mathfrak{c}} \mathbf{M}\left(\mathfrak{f}^{-1} \mathfrak{c}\right) \mathbf{N} \mathfrak{N}\left(\mathfrak{a}_{2} \mathfrak{f}\right)^{-\beta}
$$


where $c=\operatorname{ann}_{\mathcal{O}}\left(\lambda_{1}+\lambda_{2}\right)$. Thus we find

$$
\varphi_{\beta}\left(f_{2} f_{1}\right)=\left(\frac{\mathbf{N} a_{2}}{\mathbf{N} a_{1}}\right)^{-\beta} \varphi_{\beta}\left(f_{1} f_{2}\right) .
$$

We already know that both sides vanish unless $\mathfrak{a}_{1}=\mathfrak{b}_{2}$, so we get

$$
\varphi_{\beta}\left(f_{2} f_{1}\right)=\left(\frac{\mathbf{N a}}{\mathbf{N b}_{2}}\right)^{-\beta} \varphi_{\beta}\left(f_{1} f_{2}\right) .
$$

Now we have for all $t \in \mathbb{R}$,

$$
\sigma_{t}\left(f_{2}\right)=\sigma_{t}\left(\mu_{\mathfrak{a}_{2}} e\left(\psi^{2}, \lambda_{2}\right) \mu_{\mathfrak{b}_{2}}^{*}\right)=\mathbf{N a} \mathfrak{a}_{2}^{i t} \mu_{\mathfrak{a}_{2}} e\left(\psi^{2}, \lambda_{2}\right) \mathbf{N b}_{2}^{-i t} \mu_{\mathfrak{b}_{2}}^{*}=\left(\frac{\mathbf{N a} \mathfrak{a}_{2}}{\mathbf{N b}_{2}}\right)^{i t} f_{2} .
$$

Thus, letting

$$
F_{\beta, f_{1}, f_{2}}(z)=\left(\frac{\mathbf{N} \mathfrak{a}_{2}}{\mathbf{N} \mathfrak{b}_{2}}\right)^{i z} \varphi_{\beta}\left(f_{1} f_{2}\right)
$$

defines a bounded holomorphic function $F_{\beta, f_{1}, f_{2}}$ on the strip, realizing the $\mathrm{KMS}_{\beta}$ property for the state $\varphi_{\beta}$ and the pair $\left(f_{1}, f_{2}\right)$.

Proposition 4.1.3. Let $\beta \in \mathbb{R}_{+}^{*}$.

(1) The state $\varphi_{\beta}$ on $C_{1}$ is the only $K M S_{\beta}$ state of $\left(C_{1},\left(\sigma_{t}\right)\right)$.

(2) The state $\varphi_{\beta}$ on $C_{k, \infty}$ is the only Galois-invariant $K M S_{\beta}$ state of $\left(C_{k, \infty},\left(\sigma_{t}\right)\right)$.

Proof. Clearly, the two statements are equivalent. Let us prove (1). Let $\varphi$ be a $\operatorname{KMS}_{\beta}$ state of $\left(C_{1},\left(\sigma_{t}\right)\right)$. Let us show that

$$
\varphi=\varphi_{\beta}
$$

Let $\mathfrak{a}, \mathfrak{b} \in \mathfrak{I}_{\mathcal{O}}$. We have

$$
\varphi\left(\mu_{\mathfrak{a}} \mu_{\mathfrak{b}}^{*}\right)=\varphi\left(\mu_{\mathfrak{b}}^{*} \sigma_{i \beta}\left(\mu_{\mathfrak{a}}\right)\right)=\mathbf{N a}{ }^{-\beta} \varphi\left(\mu_{\mathfrak{b}}^{*} \mu_{\mathfrak{a}}\right) .
$$

Let us first work in the case when $\mathfrak{a} \neq \mathfrak{b}$. Let us prove that $\varphi\left(\mu_{\mathfrak{a}} \mu_{\mathfrak{b}}^{*}\right)=0$. Since $\varphi\left(\mu_{\mathfrak{a}} \mu_{\mathfrak{b}}^{*}\right)=\overline{\varphi\left(\mu_{\mathfrak{b}} \mu_{\mathfrak{a}}^{*}\right)}$, we may swap $\mathfrak{a}$ and $\mathfrak{b}$, and therefore we may assume without loss of generality that $a \nmid \mathfrak{b}$. Let $\mathfrak{b}=\mathfrak{a}+\mathfrak{b}$ denote the gcd of $a$ and $\mathfrak{b}$. We have

$$
\mu_{\mathfrak{b}}^{*} \mu_{\mathfrak{a}}=\mu_{\mathfrak{D}^{-1} \mathfrak{b}}^{*} \mu_{\mathfrak{b}}^{*} \mu_{\mathfrak{D}} \mu_{\mathfrak{D}^{-1} \mathfrak{a}}=\mu_{\mathfrak{D}^{-1} \mathfrak{b}}^{*} \mu_{\mathfrak{D}^{-1} \mathfrak{a}} .
$$

Since $\mathfrak{D}^{-1} \mathfrak{a}$ and $\mathfrak{D}^{-1} \mathfrak{b}$ are relatively prime, we have

$$
\mu_{\mathfrak{D}^{-1} \mathfrak{b}}^{*} \mu_{\mathfrak{D}^{-1} \mathfrak{a}}=\mu_{\mathfrak{D}^{-1} \mathfrak{a}} \mu_{\mathfrak{D}^{-1} \mathfrak{b}}^{*} .
$$


Thus, equation (50) applied to $\mathfrak{b}^{-1} \mathfrak{a}$ and $\mathfrak{D}^{-1} \mathfrak{b}$ gives

$$
\varphi\left(\mu_{\mathfrak{D}^{-1} \mathfrak{a}} \mu_{\mathfrak{D}^{-1} \mathfrak{b}}^{*}\right)=\mathbf{N}\left(\mathfrak{D}^{-1} \mathfrak{a}\right)^{-\beta} \varphi_{\beta}\left(\mu_{\mathfrak{D}^{-1} \mathfrak{a}} \mu_{\mathfrak{D}^{-1} \mathfrak{b}}^{*}\right) .
$$

As $a \nmid \mathfrak{b}$, we have $\mathfrak{D}^{-1} \mathfrak{a} \neq 1$, so equation (53) gives

$$
\varphi\left(\mu_{\mathfrak{D}^{-1} \mathfrak{a}} \mu_{\mathfrak{D}^{-1} \mathfrak{b}}^{*}\right)=0 .
$$

Hence equation (52) gives $\varphi\left(\mu_{\mathfrak{d}^{-1} \mathfrak{b}_{\mathfrak{b}}}^{*} \mu_{\mathfrak{D}^{-1} \mathfrak{a}}\right)=0$, so equation (51) gives $\varphi\left(\mu_{\mathfrak{b}}^{*} \mu_{\mathfrak{a}}\right)=0$, so equation (50) gives $\varphi\left(\mu_{\mathfrak{a}} \mu_{\mathfrak{b}}^{*}\right)=0$. Hence we have proven that

$$
\mathfrak{a} \neq \mathfrak{b} \Longrightarrow \varphi\left(\mu_{\mathfrak{a}} \mu_{\mathfrak{b}}^{*}\right)=0=\varphi_{\beta}\left(\mu_{\mathfrak{a}} \mu_{\mathfrak{b}}^{*}\right) .
$$

In the case when $\mathfrak{a}=\mathfrak{b}$ equation (50) gives

$$
\varphi\left(\mu_{\mathfrak{a}} \mu_{\mathfrak{a}}^{*}\right)=\mathbf{N a}^{-\beta} \varphi\left(\mu_{\mathfrak{a}}^{*} \mu_{\mathfrak{a}}\right)=\mathbf{N a}^{-\beta}=\varphi_{\beta}\left(\mu_{\mathfrak{a}} \mu_{\mathfrak{a}}^{*}\right) .
$$

Thus we have proven that

$$
\varphi\left(\mu_{\mathfrak{a}} \mu_{\mathfrak{b}}^{*}\right)=\varphi_{\beta}\left(\mu_{\mathfrak{a}} \mu_{\mathfrak{b}}^{*}\right) \quad \text { for all } \mathfrak{a}, \mathfrak{b} \in \mathfrak{I}_{\mathcal{O}} .
$$

As the linear span of the $\mu_{\mathfrak{a}} \mu_{\mathfrak{b}}^{*}$ is the $*$-algebra generated by the $\mu_{\mathfrak{a}}$, it is dense in $C_{1}$ (by Definition 3.5.3), so we get $\varphi=\varphi_{\beta}$.

4.2. Action of $\operatorname{Gal}(K / \boldsymbol{k})$ on extremal $\mathrm{KMS}_{\boldsymbol{\beta}}$ states. As usual $\mathrm{Gal}(K / k)$ is endowed with its profinite topology. It acts on the set of states by $(\sigma, \varphi) \mapsto \varphi \circ \sigma$. Obviously the $\mathrm{KMS}_{\beta}$ condition and factoriality are preserved by this action. Hence, the sets $K_{\beta}$ and $\mathscr{E}\left(K_{\beta}\right)$ are invariant under the action of $\mathrm{Gal}(K / k)$.

The proof of the next proposition comes from that of Theorem 25 in [3].

Proposition 4.2.1. For any $\beta \in \mathbb{R}_{+}^{*}$, the action of $\operatorname{Gal}(K / k)$ on $\mathcal{E}\left(K_{\beta}\right)$ is transitive.

Proof. The main ingredient is that the Galois-fixed subalgebra has a unique $\mathrm{KMS}_{\beta}$ state (cf. Proposition 4.1.3). As in the proof of Lemma 3.5.2, let $d \sigma$ be the normalized Haar measure on $\operatorname{Gal}(K / k)$, and let $\mathbf{E}$ denote the map defined in equation (41).

Let $\varphi_{1}, \varphi_{2} \in \mathcal{E}\left(K_{\beta}\right)$. Then $\varphi_{1} \circ \mathbf{E}$ and $\varphi_{2} \circ \mathbf{E}$ are Galois-invariant elements of $K_{\beta}$. Thus, by Proposition 4.1.3, they are equal:

$$
\varphi_{1} \circ \mathbf{E}=\varphi_{2} \circ \mathbf{E} .
$$

But we have, for $i=1,2$,

$$
\varphi_{i} \circ \mathbf{E}=\int_{\mathrm{Gal}(K / k)} \varphi_{i} \circ \sigma d \sigma .
$$

Equation (54) gives two decompositions of the same state as a barycenter of extremal $\mathrm{KMS}_{\beta}$ states, but such a decomposition is unique (cf. [4], II, Theorem 5.3.30), so the orbits of $\varphi_{1}$ and of $\varphi_{2}$ under $\operatorname{Gal}(K / k)$ are the same one. 
Let $S$ denote the space of all states of $C_{k, \infty}$, endowed with the weak* topology. Recall that the weak* topology on $S$ is the one for which a basis of open neighborhoods of a state $\varphi_{0}$ is given by the

$$
B\left(\varphi_{0} ; x_{1}, \ldots, x_{n} ; \varepsilon\right)=\left\{\varphi \in S|| \varphi\left(x_{i}\right)-\varphi_{0}\left(x_{i}\right) \mid<\varepsilon \text { for all } i\right\}
$$

for all $n \geqslant 1, x_{1}, \ldots, x_{n} \in C_{k, \infty}$ and $\varepsilon>0$.

Lemma 4.2.2. The action of $\operatorname{Gal}(K / k)$ on $S$, given by $(\sigma, \varphi) \mapsto \varphi \circ \sigma$, is continuous.

Proof. Let $\varphi_{0} \in S, n \leqslant 1$, let $x_{1}, \ldots, x_{n} \in C_{k, \infty}$, and let $\varepsilon>0$. Let $U=B\left(\varphi_{0} ; x_{1}, \ldots, x_{n} ; \varepsilon\right)$, as defined in equation (55). Let us find an open set $V \subset \operatorname{Gal}(K / k)$ and an open set $W \subset S$ such that

$$
\varphi \circ \sigma \in U \text { for all } \sigma \in V, \varphi \in W \text {. }
$$

Let us take $W=B\left(\varphi_{0} \in S ; x_{1}, \ldots, x_{n} ; \varepsilon / 2\right)$. By Proposition 3.4.2, for any $i$, $1 \leqslant i \leqslant n$, the map

$$
\begin{aligned}
\operatorname{Gal}(K / k) & \rightarrow C_{k, \infty}, \\
\sigma & \mapsto \sigma\left(x_{i}\right)
\end{aligned}
$$

is continuous, so the finite intersection

$$
V=\bigcap_{i=1}^{n}\left\{\sigma \in \operatorname{Gal}(K / k) \mid\left\|\sigma\left(x_{i}\right)-x_{i}\right\|<\varepsilon / 2\right\}
$$

is an open neighborhood of 1 in $\operatorname{Gal}(K / k)$. Hence, all we have to do is to check equation (56). Let $\sigma \in V$ and $\varphi \in W$. Let $1 \leqslant i \leqslant n$. We have $\left\|\sigma\left(x_{i}\right)-x_{i}\right\|<\varepsilon / 2$, so, as $\varphi$ is a state, $\left|\varphi\left(\sigma\left(x_{i}\right)\right)-\varphi\left(x_{i}\right)\right|<\varepsilon / 2$. On the other hand, as $\varphi \in W$, we have $\left|\varphi\left(x_{i}\right)-\varphi_{0}\left(x_{i}\right)\right|<\varepsilon / 2$. Thus $\left|\varphi\left(\sigma\left(x_{i}\right)\right)-\varphi_{0}\left(x_{i}\right)\right|<\varepsilon$, so $\varphi \circ \sigma \in U$.

\subsection{Extremal $\mathrm{KMS}_{\beta}$ states at low temperature $1 / \beta<1$ and special values.} Recall that $X^{\mathrm{adm}}$ is the subspace of $X$ of admissible elements, that $\mathcal{E}\left(K_{\beta}\right)$ is endowed with the weak* topology, and that $\operatorname{Gal}(K / k)$ is endowed with its profinite topology. In this subsection, for any $\beta>1$, we shall construct a homeomorphism $X^{\text {adm }} \rightarrow \mathcal{E}\left(K_{\beta}\right)$, $\chi \mapsto \varphi_{\beta, \chi}$, commuting with the actions of $\operatorname{Gal}(K / k)$, and we shall show that both $\mathcal{E}\left(K_{\beta}\right)$ (for $\beta>1$ ) and $X^{\text {adm }}$ are principal homogeneous spaces under $\operatorname{Gal}(K / k)$. Moreover, we shall compute the values of $\varphi_{\beta, \chi}$ at certain elements of $\mathscr{H}$ and relate them to special values of partial zeta functions of $k$.

For any $\chi \in X^{\mathrm{adm}}$, as at the beginning of the proof of Lemma 3.7.1, let us make the identification $\mathscr{E}_{\chi}=\mathfrak{F}_{\chi}=\mathfrak{S}_{\mathcal{O}}$ so that $\pi_{\chi}$ is seen as a representation in $\ell^{2}\left(\mathfrak{I}_{\mathcal{O}}\right)$. Let $\left(\varepsilon_{\mathfrak{a}}\right)_{\mathfrak{a} \in \mathfrak{I}_{\mathcal{O}}}$ be the standard orthonormal basis of $\ell^{2}\left(\mathfrak{I}_{\mathcal{O}}\right)$. 
Definition 4.3.1. Let $H$ be the unbounded operator on $\ell^{2}\left(\widetilde{I}_{\mathcal{O}}\right)$ defined by

$$
H \varepsilon_{\mathfrak{a}}=(\log \mathbf{N a}) \varepsilon_{\mathfrak{a}} \quad \text { for all } \mathfrak{a} \in \mathfrak{s}_{\mathcal{O}}
$$

Lemma 4.3.2. For any $\chi \in X^{\mathrm{adm}}$, for all $t \in \mathbb{R}$ and for all $f \in C_{k, \infty}$, we have

$$
\pi_{\chi}\left(\sigma_{t}(f)\right)=e^{i t H} \pi_{\chi}(f) e^{-i t H} .
$$

Proof. By Lemma 2.2.3, it is enough to do the proof in the case when $f \in C_{c}(\mathcal{G})$. It is then a straightforward computation.

The function $\beta \mapsto \operatorname{Tr}\left(e^{-\beta H}\right)$ is trivially computed:

Lemma 4.3.3. For all $\beta>1$, we have $\operatorname{Tr}\left(e^{-\beta H}\right)=\zeta_{k, \infty}(\beta)$.

Proof. $\operatorname{Tr}\left(e^{-\beta H}\right)=\sum_{\mathfrak{a} \in \mathfrak{I}_{\mathcal{O}}} e^{-\beta \log \mathbf{N a}}=\sum_{\mathfrak{a} \in \mathfrak{I}_{\mathcal{O}}} \mathbf{N a} a^{-\beta}=\zeta_{k, \infty}(\beta)$.

Definition 4.3.4. For any $\chi \in X^{\text {adm }}$, for any $\beta>1$, we define a linear functional $\varphi_{\beta, \chi}$ on $C_{k, \infty}$ by

$$
\varphi_{\beta, \chi}(f)=\zeta_{k, \infty}(\beta)^{-1} \operatorname{Tr}\left(\pi_{\chi}(f) e^{-\beta H}\right)
$$

Let $\left(\varepsilon_{\mathfrak{a}}\right)_{\mathfrak{a} \in \mathfrak{I}_{\mathcal{O}}}$ denote the standard basis of $\ell^{2}\left(\mathfrak{I}_{\mathcal{O}}\right)$.

Lemma 4.3.5. For any $\chi \in X^{\mathrm{adm}}$ and for any $\beta>1, \varphi_{\beta, \chi}$ is a $K M S_{\beta}$ state of the $C^{*}$-dynamical system $\left(C_{k, \infty},\left(\sigma_{t}\right)\right)$.

Proof. By Lemma 4.3.3, we have $\varphi_{\beta, \chi}(1)=1$. We also have, for any $f \in C_{k, \infty}$,

$$
\varphi_{\beta, \chi}\left(f f^{*}\right)=\zeta_{k, \infty}(\beta)^{-1} \operatorname{Tr}\left(\pi_{\chi}\left(f^{*}\right) e^{-\beta H} \pi_{\chi}(f)\right) \geqslant 0,
$$

so $\varphi_{\beta, \chi}$ is a state on $C_{k, \infty}$. For any $f, f^{\prime} \in C_{k, \infty}$, let us define a bounded continuous function $F_{\beta, \chi, f, f^{\prime}}$ on the strip $\{z \in \mathbb{C} \mid 0 \leqslant \operatorname{Im} z \leqslant \beta\}$ by

$$
F_{\beta, \chi, f, f^{\prime}}(z)=\zeta_{k, \infty}(\beta)^{-1} \operatorname{Tr}\left(e^{-\beta H} \pi_{\chi}(f) e^{i z H} \pi_{\chi}\left(f^{\prime}\right) e^{-i z H}\right) .
$$

One checks that the restriction of $F_{\beta, \chi, f, f^{\prime}}$ to $\{z \in \mathbb{C} \mid 0<\operatorname{Im} z<\beta\}$ is holomorphic. By Lemma 4.3.2, we have, for all $t \in \mathbb{R}$,

$$
F_{\beta, \chi, f, f^{\prime}}(t)=\varphi_{\beta, \chi}\left(f \sigma_{t}\left(f^{\prime}\right)\right) \quad \text { and } \quad F_{\beta, \chi, f, f^{\prime}}(t+i \beta)=\varphi_{\beta, \chi}\left(\sigma_{t}\left(f^{\prime}\right) f\right) .
$$

So $\varphi_{\beta, \chi}$ is a $\mathrm{KMS}_{\beta}$ state of $\left(C_{k, \infty},\left(\sigma_{t}\right)\right)$.

Lemma 4.3.6. For any $\chi \in X^{\mathrm{adm}}$, for any $\beta>1$, for any $\sigma \in \mathrm{Gal}(K / k)$, we have

$$
\varphi_{\beta, \sigma \chi}=\varphi_{\beta, \chi} \circ \sigma \text {. }
$$


Proof. By definition of $\varphi_{\beta, \sigma \chi}$, it is enough to check that $\pi_{\sigma \chi}(f)=\pi_{\chi}(\sigma f)$. By Proposition 3.3.5, it is enough to prove it when $f$ is one of the $e(\psi, \lambda)$ or one of the $\mu_{\mathfrak{a}}$. The result then follows from equations (38), (39).

Lemma 4.3.7. For any $\chi \in X^{\mathrm{adm}}$ and for any $\beta>1$, the GNS representation of $\varphi_{\beta, \chi}$ is $\left(\pi_{\beta, \chi}, \Omega_{\beta, \chi}\right)$, where $\pi_{\beta, \chi}: C_{k, \infty} \rightarrow B\left(\ell^{2}\left(\widetilde{\Im}_{\mathcal{O}}\right) \otimes \ell^{2}\left(\widetilde{\Im}_{\mathcal{O}}\right)\right)$ is given by

$$
\pi_{\beta, \chi}(f)(\xi \otimes \eta)=\pi_{\chi}(f) \xi \otimes \eta,
$$

and the cyclic vector $\Omega_{\beta, \chi} \in \ell^{2}\left(\mathfrak{I}_{\mathcal{O}}\right) \otimes \ell^{2}\left(\mathfrak{I}_{\mathcal{O}}\right)$ is given by

$$
\Omega_{\beta, \chi}=\zeta_{k, \infty}(\beta)^{-1 / 2} \sum_{\mathfrak{a} \in \Im_{\mathcal{O}}} \mathbf{N} a^{-\beta / 2} \varepsilon_{\mathfrak{a}} \otimes \varepsilon_{\mathfrak{a}} .
$$

Proof. We obviously have

$$
\varphi_{\beta, \chi}(f)=\left\langle\pi_{\beta, \chi}(f) \Omega_{\beta, \chi}, \Omega_{\beta, \chi}\right\rangle .
$$

Hence, we only have to show that $\Omega_{\beta, \chi}$ is a cyclic vector for $\pi_{\beta, \chi}$. For any maximal ideal $\mathfrak{p}$ of $\mathcal{O}$ and any $n \geqslant 0$, using equation(45), we find

$$
\pi_{\chi}\left(\mu_{\mathfrak{p}^{n}}^{*}\right) \varepsilon_{\mathfrak{a}}=1_{\mathfrak{p}^{n} \mid \mathfrak{a}} \varepsilon_{\mathfrak{p}^{-n} \mathfrak{a}} \quad \text { for all } \mathfrak{a} \in \mathfrak{I}_{\mathcal{O}}
$$

and hence

$$
\pi_{\chi}\left(\mu_{\mathfrak{p}^{n}} \mu_{\mathfrak{p}^{n}}^{*}\right) \varepsilon_{\mathfrak{a}}=1_{\mathfrak{p}^{n} \mid \mathfrak{a}} \varepsilon_{\mathfrak{a}} \quad \text { for all } \mathfrak{a} \in \mathfrak{I}_{\mathcal{O}} .
$$

Thus, if we let $v_{\mathfrak{p}^{n}}=\mu_{\mathfrak{p}^{n}} \mu_{\mathfrak{p}^{n}}^{*}-\mu_{\mathfrak{p}^{n+1}} \mu_{\mathfrak{p}^{n+1}}^{*}$ we get

$$
\pi_{\chi}\left(v_{\mathfrak{p}^{n}}\right) \varepsilon_{\mathfrak{a}}=1_{\mathfrak{p}^{n} \mid \mathfrak{a} \text { and } \mathfrak{p}^{n+1} \nmid \mathfrak{a}} \varepsilon_{\mathfrak{a}} \quad \text { for all } \mathfrak{a} \in \mathfrak{I}_{\mathcal{O}} .
$$

Now let $\mathfrak{b} \in \mathfrak{I}_{\mathcal{O}}$. Let us show that $\varepsilon_{\mathfrak{b}} \otimes \varepsilon_{\mathfrak{b}}$ is in the closure of $\pi_{\beta, \chi}\left(C_{k, \infty}\right)\left(\Omega_{\beta, \chi}\right)$. Write $\mathfrak{b}=\prod_{\mathfrak{p}} \mathfrak{p}^{n_{\mathfrak{p}}}$ with $n_{\mathfrak{p}} \geqslant 0$. For $T>0$, let $P_{T}$ denote the set of all maximal ideals $\mathfrak{p}$ with $\mathbf{N} \mathfrak{p}<T$. The family $\left(P_{T}\right)_{T}$ is a growing family of finite sets whose union is the set of all maximal ideals of $\mathcal{O}$. For all $T$, let $v_{T} \in C_{k, \infty}$ be defined by

$$
v_{T}=\prod_{\mathfrak{p} \in P_{T}} v_{\mathfrak{p}^{n}} \text {. }
$$

We have

$$
\pi_{\beta, \chi}\left(v_{T}\right)\left(\Omega_{\beta, \chi}\right)=\zeta_{k, \infty}(\beta)^{-1 / 2} \sum_{\mathfrak{a} \in Q_{T}} \mathbf{N a} a^{-\beta / 2} \varepsilon_{\mathfrak{a}} \otimes \varepsilon_{\mathfrak{a}},
$$

where $Q_{T}$ is the set of all $\mathfrak{a} \in \mathfrak{I}_{\mathcal{O}}$ such that for all $\mathfrak{p} \in P_{T}$, the $\mathfrak{p}$-adic valuations of $\mathfrak{a}$ and $\mathfrak{b}$ are equal. Since the series $\sum_{\mathfrak{a}} \mathbf{N a}^{-\beta}$ is convergent, we see that

$$
\pi_{\beta, \chi}\left(v_{T}\right)\left(\Omega_{\beta, \chi}\right) \stackrel{T \rightarrow+\infty}{\longrightarrow} \zeta_{k, \infty}(\beta)^{-1 / 2} \mathbf{N b}^{-\beta / 2} \varepsilon_{\mathfrak{b}} \otimes \varepsilon_{\mathfrak{b}} .
$$

Thus, we have shown that $\varepsilon_{\mathfrak{b}} \otimes \varepsilon_{\mathfrak{b}}$ is in the closure of $\pi_{\beta, \chi}\left(C_{k, \infty}\right)\left(\Omega_{\beta, \chi}\right)$. Applying the $\pi_{\beta, \chi}\left(\mu_{\mathfrak{a}}\right)$ and the $\pi_{\beta, \chi}\left(\mu_{\mathfrak{a}}^{*}\right)$ to that shows that for all $\mathfrak{b}_{1}, \mathfrak{b}_{2} \in \mathfrak{I}_{\mathcal{O}}$, the element $\varepsilon_{\mathfrak{b}_{1}} \otimes \varepsilon_{\mathfrak{b}_{2}}$ is in the closure of $\pi_{\beta, \chi}\left(C_{k, \infty}\right)\left(\Omega_{\beta, \chi}\right)$. 
Proposition 4.3.8. For any $\chi \in X^{\mathrm{adm}}$ and for any $\beta>1$, the state $\varphi_{\beta, \chi}$ is factorial (hence extremal) of type $\mathrm{I}_{\infty}$.

Proof. Let $A$ denote the weak closure of $\pi_{\beta, \chi}\left(C_{k, \infty}\right)$ in $B\left(\ell^{2}\left(\widetilde{I}_{\mathcal{O}}\right) \otimes \ell^{2}\left(\mathfrak{I}_{\mathcal{O}}\right)\right)$. By Lemma 3.7.1, the representation $\pi_{\chi}$ is irreducible. Thus inside $B \ell^{2}\left(\mathfrak{\Im}_{\mathcal{O}}\right)$, we have $\pi_{\chi}\left(C_{k, \infty}\right)^{\prime}=\mathbb{C}$. Using Takesaki [33], I, Chapter IV, Proposition 1.6 (i), we deduce that inside $B\left(\ell^{2}\left(\widetilde{\mathfrak{I}}_{\mathcal{O}}\right) \otimes \ell^{2}\left(\widetilde{\mathfrak{I}}_{\mathcal{O}}\right)\right)$, we have

$$
\pi_{\beta, \chi}\left(C_{k, \infty}\right)^{\prime}=\mathbb{C} \otimes B \ell^{2}\left(\widetilde{\Im}_{\mathcal{O}}\right) .
$$

Thus, using [33], I, Chapter IV, Proposition 1.6(ii), we deduce

$$
A=\pi_{\beta, \chi}\left(C_{k, \infty}\right)^{\prime \prime}=B \ell^{2}\left(\mathfrak{\Im}_{\mathcal{O}}\right) \otimes \mathbb{C} .
$$

In particular, we have $A \simeq B \ell^{2}\left(\widetilde{\Im}_{\mathcal{O}}\right)$, so $A$ is a factor of type $\mathrm{I}_{\infty}$.

Lemma 4.3.9. For any $\beta>1$, the map $X^{\mathrm{adm}} \rightarrow \mathcal{E}\left(K_{\beta}\right), \chi \mapsto \varphi_{\beta, \chi}$, is injective.

Proof. We reuse the notations of the proof of the previous lemma. Let us extend $\varphi_{\beta, \chi}$ to a state $\widetilde{\varphi}_{\beta, \chi}$ on the von Neumann algebra $A=B \ell^{2}\left(\widetilde{I}_{\mathcal{O}}\right) \otimes \mathbb{C}$ by

$$
\tilde{\varphi}_{\beta, \chi}(a \otimes 1)=\left\langle a\left(\Omega_{\beta, \chi}\right), \Omega_{\beta, \chi}\right\rangle \quad \text { for all } a \in B \ell^{2}\left(\widetilde{\Im}_{\mathcal{O}}\right) .
$$

For any $\hat{\beta}>0$, we have $e^{-\hat{\beta} H} \in B \ell^{2}\left(\widetilde{\mathfrak{S}}_{\mathcal{O}}\right)$. We have, for all $\psi \in H($ sgn) and for all $\lambda \in \psi\left(\mathbf{C}_{\infty}\right)^{\text {tor }}:$

$\zeta_{k, \infty}(\beta) \lim _{\hat{\beta} \rightarrow+\infty} \tilde{\varphi}_{\beta, \chi}\left(\pi_{\chi}(e(\psi, \lambda)) e^{-\hat{\beta} H} \otimes 1\right)=\left\langle\pi_{\chi}(e(\psi, \lambda))\left(\varepsilon_{1}\right), \varepsilon_{1}\right\rangle=1_{\chi \in X_{\psi}} \chi(\lambda)$.

Thus, $\chi$ is uniquely determined.

We can now prove the main result classifying extremal $\mathrm{KMS}_{\beta}$ states at low temperature. Recall that $\operatorname{Gal}(K / k)$ is endowed with its profinite topology, and $\mathscr{E}\left(K_{\beta}\right)$ is endowed with the weak* topology.

Theorem 4.3.10. For any $\beta>1$, the topological space $\mathcal{E}\left(K_{\beta}\right)$ is principal homogeneous under $\operatorname{Gal}(K / k)$.

Proof. We must show that for any $\varphi \in \mathcal{E}\left(K_{\beta}\right)$, the map $\operatorname{Gal}(K / k) \rightarrow \mathcal{E}\left(K_{\beta}\right)$, $\sigma \mapsto \varphi \circ \sigma$, is a homeomorphism. We already know that it is surjective (Proposition 4.2.1) and continuous (Lemma 4.2.2). Thus, as $\operatorname{Gal}(K / k)$ is compact, it only remains to show that it is injective. Let $\varphi \in \mathcal{E}\left(K_{\beta}\right)$ and $\sigma \in \mathrm{Gal}(K / k)$ such that $\varphi \circ \sigma=\varphi$. We have to show that $\sigma=1$. Let $\chi \in X^{\mathrm{adm}}$. By Proposition 4.3.8 we have $\varphi_{\beta, \chi} \in \mathscr{E}\left(K_{\beta}\right)$. By Proposition 4.2.1, there exists $\tau \in \operatorname{Gal}(K / k)$ such that $\varphi=\varphi_{\beta, \chi} \circ \tau$. By Lemma 4.3.6 we have $\varphi=\varphi_{\beta, \tau \chi}$ and $\varphi \circ \sigma=\varphi_{\beta, \sigma \tau \chi}$, so $\varphi_{\beta, \sigma \tau \chi}=\varphi_{\beta, \tau \chi}$. By Lemma 4.3.9, we deduce $\sigma \tau \chi=\tau \chi$. By Proposition 3.6.9 we find $\sigma \tau=\tau$, so $\sigma=1$. 
Theorem 4.3.11. For any $\beta>1$, the map $X^{\mathrm{adm}} \rightarrow \mathcal{E}\left(K_{\beta}\right), \chi \mapsto \varphi_{\beta, \chi}$, is a homeomorphism.

Proof. It is injective by Lemma 4.3.9. Let us check surjectivity. Let $\varphi \in \mathcal{E}\left(K_{\beta}\right)$ and let $\chi_{0} \in X^{\mathrm{adm}}$. By Proposition 4.2.1 and Lemma 4.3.6, there exists $\sigma \in \operatorname{Gal}(K / k)$ such that $\varphi=\varphi_{\beta, \sigma \chi_{0}}$. Thus, the map $\chi \mapsto \varphi_{\beta, \chi}$ is bijective. One checks that it is continuous. By definition of an admissible character, $X^{\mathrm{adm}}$ is a closed subspace of $X$. Thus $X^{\text {adm }}$ is compact, so the considered map is a homeomorphism.

Relations between certain special values of $\mathrm{KMS}_{\boldsymbol{\beta}}$ states and of partial zeta functions. Let us now compute the values of the states $\varphi_{\beta, \chi}$ on some of the generators $e(\phi, \lambda)$. Let $A_{+}$denote the subset of $\mathfrak{s}_{\mathcal{O}}$ of all ideals a such that $\sigma_{\mathfrak{a}}=1$, where $\sigma_{\mathfrak{a}}=\left(a, H^{+} / k\right) \in \operatorname{Gal}\left(H^{+} / k\right)$ is the Artin automorphism of $H^{+}$associated to a. For any $\mathfrak{c} \in \mathfrak{I}_{\mathcal{O}}$ and any $\sigma \in \operatorname{Gal}\left(K_{\mathfrak{c}} / k\right)$, let $A_{\mathfrak{c}, \sigma}$ denote the subset of $A_{+}$of all ideals a prime to $c$ and such that $\sigma_{\mathfrak{a}}=\sigma$, where $\sigma_{\mathfrak{a}}=\left(a, K_{\mathfrak{c}} / k\right) \in \operatorname{Gal}\left(K_{\mathfrak{c}} / k\right)$ is the Artin automorphism of $K_{\mathfrak{c}}$ associated to a. Note that $A_{+}$and the $A_{\mathfrak{c}, \sigma}$ are generalized ideal classes of $\mathcal{O}$.

Let $\zeta_{k, \infty}^{+}$and $\zeta_{k, \infty}^{\mathfrak{c}, \sigma}$ (for any $c \in \mathfrak{S}_{\mathcal{O}}$ and $\sigma \in \operatorname{Gal}\left(K_{\mathfrak{c}} / H^{+}\right)$) be the partial zeta functions associated to $A_{+}$and $A_{\mathfrak{c}, \sigma}$, respectively:

$$
\begin{aligned}
& \zeta_{k, \infty}^{+}(\beta)=\sum_{\mathfrak{a} \in A_{+}} \mathbf{N a}^{-\beta}, \\
& \zeta_{k, \infty}^{\mathfrak{c}, \sigma}(\beta)=\sum_{\mathfrak{a} \in A_{\mathfrak{c}, \sigma}} \mathbf{N a}^{-\beta} .
\end{aligned}
$$

Theorem 4.3.12. Let $\beta>1, \phi \in H(\operatorname{sgn})$, and $\chi \in X^{\mathrm{adm}} \cap X_{\phi}$.

(1) We have

$$
\varphi_{\beta, \chi}(e(\phi, 0))=\frac{\zeta_{k, \infty}^{+}(\beta)}{\zeta_{k, \infty}(\beta)}
$$

(2) For any maximal ideal $\mathfrak{p}$ of $\mathcal{O}$, for any $\lambda \in \phi[\mathfrak{p}]$, we have

$$
\varphi_{\beta, \chi}(e(\phi, \lambda))=\zeta_{k, \infty}(\beta)^{-1}\left(\mathbf{N p}^{-\beta} \zeta_{k, \infty}^{+}(\beta)+\sum_{\sigma \in \operatorname{Gal}\left(K_{\mathfrak{p}} / H^{+}\right)} \chi(\sigma \lambda) \zeta_{k, \infty}^{\mathfrak{p}, \sigma}(\beta)\right) .
$$

Proof. Let us first prove (1). By definition, $A_{+}$is the subset of $\mathfrak{I}_{\mathcal{O}}$ of all ideals a such that $\sigma_{\mathfrak{a}}=1$, where $\sigma_{\mathfrak{a}}=\left(\mathfrak{a}, H^{+} / k\right) \in \operatorname{Gal}\left(H^{+} / k\right)$ is the Artin automorphism of $H^{+}$associated to $a$. Hence, by Theorem 1.3.9, we have

$$
A_{+}=\left\{a \in \mathfrak{I}_{\mathcal{O}} \mid a * \phi=\phi\right\}=\left\{a \in \mathfrak{I}_{\mathcal{O}} \mid a^{-1} * \phi=\phi\right\}=\left\{a \in \mathfrak{I}_{\mathcal{O}} \mid \chi^{a} \in X_{\phi}\right\} .
$$


Thus, by definition of $\varphi_{\beta, \chi}$, for any $\lambda \in \phi\left(\mathbf{C}_{\infty}\right)^{\text {tor }}$, we have

$$
\begin{aligned}
\varphi_{\beta, \chi}(e(\phi, \lambda)) & =\zeta_{k, \infty}(\beta)^{-1} \sum_{\mathfrak{a} \in \mathfrak{I}_{\mathcal{O}}} 1_{\chi^{\mathfrak{a} \in X_{\phi}}} \chi^{\mathfrak{a}}(\lambda) \mathbf{N a}{ }^{-\beta} \\
& =\zeta_{k, \infty}(\beta)^{-1} \sum_{\mathfrak{a} \in A_{+}} \chi\left(\phi_{\mathfrak{a}}(\lambda)\right) \mathbf{N a}^{-\beta}
\end{aligned}
$$

Applying this equality to $\lambda=0$ we get (1).

Let us now prove (2). Let $a \in A_{+}$. In the case when $\mathfrak{p} \mid a$, we have $\phi_{\mathfrak{a}}(\lambda)=0$, so

$$
\chi\left(\phi_{a}(\lambda)\right)=1
$$

In the case when $\mathfrak{p} \nmid a$, by Theorem 1.3.11, we have $\phi_{a}(\lambda)=\sigma_{a}(\lambda)$. Hence, equation (57) gives

$$
\begin{aligned}
& \zeta_{k, \infty}(\beta) \varphi_{\beta, \chi}(e(\phi, \lambda))=\sum_{\mathfrak{a} \in A_{+}, \mathfrak{p} \mid \mathfrak{a}} \chi\left(\phi_{\mathfrak{a}}(\lambda)\right) \mathbf{N} a^{-\beta}+\sum_{\mathfrak{a} \in A_{+}, \mathfrak{p} \mid \mathfrak{a}} \chi\left(\phi_{\mathfrak{a}}(\lambda)\right) \mathbf{N} a^{-\beta} \\
& =\sum_{\mathfrak{a} \in A_{+}, \mathfrak{p} \mid \mathfrak{a}} \mathbf{N} a^{-\beta}+\sum_{\mathfrak{a} \in A_{+}, \mathfrak{p} \mid \mathfrak{a}} \chi\left(\sigma_{\mathfrak{a}}(\lambda)\right) \mathbf{N a} a^{-\beta} \\
& =\sum_{\mathfrak{a} \in A_{+}} \mathbf{N}(\mathfrak{p a})^{-\beta}+\sum_{\sigma \in \operatorname{Gal}\left(K_{\mathfrak{p}} / H^{+}\right)} \sum_{a \in A_{\mathfrak{p}, \sigma}} \chi(\sigma \lambda) \mathbf{N a} a^{-\beta} \\
& =\mathbf{N p}^{-\beta} \zeta_{k, \infty}^{+}(\beta)+\sum_{\sigma \in \operatorname{Gal}\left(K_{\mathfrak{p}} / H^{+}\right)} \chi(\sigma \lambda) \zeta_{k, \infty}^{\mathfrak{p}, \sigma}(\beta)
\end{aligned}
$$

which proves (2).

4.4. Uniqueness of the $\mathrm{KMS}_{\boldsymbol{\beta}}$ state at high temperature $1 / \boldsymbol{\beta} \geqslant 1$. Recall that in Proposition 4.1.2, for any $\beta \in \mathbb{R}_{+}^{*}$, we found a Galois-invariant $\mathrm{KMS}_{\beta}$ state $\varphi_{\beta}$ of $\left(C_{k, \infty},\left(\sigma_{t}\right)\right)$.

In this subsection we shall prove (Theorem 4.4.15) that when $\beta \leqslant 1$, there is no other $\mathrm{KMS}_{\beta}$ state of $\left(C_{k, \infty},\left(\sigma_{t}\right)\right)$. In other words,

$$
\beta \leqslant 1 \Longrightarrow K_{\beta}=\left\{\varphi_{\beta}\right\} .
$$

Most of the ideas here come from [3], §7.

Let $\beta \in \mathbb{R}_{+}^{*}$ be such that $\beta \leqslant 1$, and let $\psi$ be a $\operatorname{KMS}_{\beta}$ state of $\left(C_{k, \infty},\left(\sigma_{t}\right)\right)$. We must show that $\psi=\varphi_{\beta}$.

Let $\overline{\mathrm{Gal}(K / k)}$ be the dual group of $\operatorname{Gal}(K / k)$. Since $\operatorname{Gal}(K / k)$ is profinite, $\overline{\operatorname{Gal}(K / k)}$ is discrete.

Let $F$ be a non-empty finite set of finite places of $k$. Recall from Definition 3.8.1 that an ideal $a \in \mathfrak{I}_{\mathcal{O}}$ is $F$-localized if all its prime divisors belong to $F$. We also 
need to define what it means to be $F$-localized for an element of $\mathrm{Gal}(K / k)$ and for an element of $C(X)$.

Let us first define what it means to be $F$-localized for an element of $\mathrm{Gal}(K / k)$. We have

so

$$
K=\lim _{\mathfrak{c} \rightarrow} K_{\mathfrak{c}},
$$

so

$$
\operatorname{Gal}(K / k)=\lim _{\leftarrow c} \operatorname{Gal}\left(K_{\mathfrak{c}} / k\right),
$$

$$
\widehat{\operatorname{Gal}(K / k)}=\lim _{\mathfrak{c} \rightarrow} \widehat{\operatorname{Gal}\left(K_{\mathfrak{c}} / k\right)} .
$$

This means that for any character $v$ of $\operatorname{Gal}(K / k)$, there exists $c \in \mathfrak{I}_{\mathcal{O}}$ such that $v$ factors through the projection $\operatorname{Gal}(K / k) \rightarrow \operatorname{Gal}\left(K_{\mathfrak{c}} / k\right)$.

Definition 4.4.1. A character $v$ of $\operatorname{Gal}(K / k)$ is said to be $F$-localized if there exists an $F$-localized ideal $c \in \mathfrak{I}_{\mathcal{O}}$ such that $v$ factors through the projection $\operatorname{Gal}(K / k) \rightarrow$ $\operatorname{Gal}\left(K_{\mathfrak{c}} / k\right)$.

Thus any $v \in \operatorname{Gal}(K / k)$ is $F$-localized for some $F$.

Let $K_{F}$ denote the extension of $H^{+}$generated by the elements of the $\phi[F]$, for $\phi \in H$ (sgn). In other words,

$$
K_{F}=\lim _{\mathfrak{c} \rightarrow} K_{\mathfrak{c}}
$$

where $c$ runs over $\mathfrak{I}_{\mathcal{O}}$. Thus a character $v$ of $\operatorname{Gal}(K / k)$ is $F$-localized if and only if it factors through the surjection

$$
\operatorname{Gal}(K / k) \rightarrow \operatorname{Gal}\left(K_{F} / k\right) .
$$

Let us now define what it means to be $F$-localized for an element of $C(X)$. For any $\phi \in H(\operatorname{sgn})$, let $\phi[F]$ denote the following subgroup of $\phi\left(\mathbf{C}_{\infty}\right)^{\text {tor }}$ :

$$
\phi[F]=\bigcup_{\mathrm{c} \text { is } F \text {-loc. }} \phi[\mathrm{c}] .
$$

Here $\mathrm{c}$ runs over all the $F$-localized ideals in $\mathfrak{\Im}_{\mathcal{O}}$. Let $X_{\phi, F}$ denote the dual group of $\phi[F]$. The restriction-to- $\phi[F]$ map is a surjective morphism

$$
X_{\phi} \rightarrow X_{\phi, F}
$$

Let $X_{F}$ denote the (disjoint) union of the $X_{\phi, F}$, for all $\phi \in H$ (sgn). The restriction maps $X_{\phi} \rightarrow X_{\phi, F}$ give a surjection

$$
X \rightarrow X_{F}
$$

This gives an injective morphism of $\mathrm{C}^{*}$-algebras

$$
C\left(X_{F}\right) \hookrightarrow C(X) .
$$

Thus, we regard $C\left(X_{F}\right)$ as a $\mathrm{C}^{*}$-subalgebra of $C(X)$. 
Definition 4.4.2. An element $f \in C(X)$ is said to be $F$-localized if it belongs to $C\left(X_{F}\right)$. In other words, $f$ is $F$-localized if, seen as a function $f: X \rightarrow \mathbb{C}$, it factors through the map $X \rightarrow X_{F}$.

Lemma 4.4.3. The $C^{*}$-algebra $C\left(X_{F}\right)$ is generated by the $e(\phi, \lambda)$, for all $\phi \in H(\mathrm{sgn})$ and all $\lambda \in \phi[F]$.

Proof. This can be checked like Lemma 3.3.4.

For any character $v$ of $\operatorname{Gal}(K / k)$, let $C_{v}$ be the following spectral subspace of $C_{k, \infty}$ :

$$
C_{v}=\left\{f \in C_{k, \infty} \mid \sigma f=v(\sigma) f \text { for all } \sigma \in \operatorname{Gal}(K / k)\right\} .
$$

Thus, when $v=1$ is the trivial character, the corresponding subspace $C_{1}$ is the Galois-fixed subalgebra computed in 3.5.2.

Lemma 4.4.4. The following subspace is dense in $C_{k, \infty}$ :

$$
\bigoplus_{v \in \operatorname{Gal}(K / k)} C_{v}
$$

Proof. Since $\operatorname{Gal}(K / k)$ is a compact abelian group of $*$-automorphisms of $C_{k, \infty}$, this follows from a result found in Pedersen [30], $\$ \$ 8.1 .4$ and 8.1.10, p. 349.

Lemma 4.4.5. The states $\psi$ and $\varphi_{\beta}$ agree on $C_{1}$.

Proof. We saw in Proposition 4.1.3 that $\left(C_{1}, \sigma_{t}\right)$ has only one $\mathrm{KMS}_{\beta}$ state. Thus, as $\psi$ and $\varphi_{\beta}$ are $\mathrm{KMS}_{\beta}$, they must agree on $C_{1}$.

Lemma 4.4.6. Suppose that for any $v \in \widehat{\operatorname{Gal}(K / k)}$ with $v \neq 1$ the state $\psi$ vanishes on the spectral subspace $C_{v}$. Then $\psi=\varphi_{\beta}$.

Proof. By Lemma 4.4.4, in order to show that $\psi$ and $\varphi_{\beta}$ are equal, it is enough to show that they agree on $C_{\nu}$ for all $\nu$. We already know that $\psi$ and $\varphi_{\beta}$ agree on $C_{1}$. As $\varphi_{\beta}$ is $\operatorname{Gal}(K / k)$-invariant, it is easy to see that it vanishes on $C_{v}$ for any non-trivial $\nu$, so we deduce that $\psi=\varphi_{\beta}$.

Thus, in order to prove that $\psi=\varphi_{\beta}$, it is enough to prove that $\psi$ vanishes on each of the spectral subspaces $C_{v}$ for $v \neq 1$. The following lemma, which is inspired by Lemma 27 (c) in [3], will be useful to show this. 
Lemma 4.4.7. Let $v \in \overline{\operatorname{Gal}(K / k)}$ with $v \neq 1$. Let $F$ be a non-empty finite set of finite places of $k$ such that $v$ is $F$-localized. Suppose that for any $F$-localized partial isometry $V \in C(X) \cap C_{\nu}$, we have

$$
\psi(V x)=0 \text { for all } x \in C_{1} .
$$

Then $\psi$ vanishes on the spectral subspace $C_{v}$.

Proof. From Theorems 4.3.10 and 4.3.11 we know that $X^{\text {adm }}$ is principal homogeneous under $\operatorname{Gal}(K / k)$. Thus, by choosing a base point $\chi_{0} \in X^{\text {adm }}$, we can identify $\operatorname{Gal}(K / k)$ with $X^{\text {adm }}$ through the map $\sigma \mapsto \sigma \chi_{0}$. Let $\mathfrak{f} \in \mathfrak{I}_{\mathcal{O}}$ be defined by

$$
\mathfrak{f}=\prod_{\mathfrak{p} \in F} \mathfrak{p} .
$$

For any $n \geqslant 1$, let $V_{n} \in C(X)$ be defined as follows. Let $\chi \in X$. If $\mathfrak{F}_{\chi}$ is of the form $\mathfrak{a}^{-1} \mathfrak{I}_{\mathcal{O}}$ with $a \mid \mathfrak{f}^{n}$, write $\chi=\sigma \chi_{0}^{a}$ with $\sigma \in \operatorname{Gal}(K / k)$ and put $V_{n}(\chi)=v(\sigma)$. Otherwise, put $V_{n}(\chi)=0$. Note that $V_{n}$ is a partial isometry and belongs to $C_{v}$. Moreover, $V_{n}$ is $F$-localized because $v$ is.

For any $\chi \in X$ we have $\left|V_{n}(\chi)\right|=1$ if $\mathfrak{F}_{\chi}$ is of the form $a^{-1} \mathfrak{I}_{\mathcal{O}}$ for some $a \mid \mathfrak{f}^{n}$ and $\left|V_{n}(\chi)\right|=0$ otherwise. As $\left|V_{n}\right|$ takes values in $\{0,1\}$, we have $\left|V_{n}\right|=\left|V_{n}\right|^{2}=V_{n} V_{n}^{*}$. Thus, by Lemmas 2.1.7 and 2.1.8, for any $\chi \in X$, we have

$$
V_{n} V_{n}^{*}(\chi)=1_{\mathfrak{F}_{\chi} \subset \mathfrak{f}^{-n} \mathfrak{\Im}_{\mathcal{O}}}=\prod_{\mathfrak{p} \in F} 1_{\mathfrak{f}}^{-n} \mathfrak{p}^{-1} \notin \mathfrak{F}_{\chi}
$$

Hence, by equation (33), we get

$$
V_{n} V_{n}^{*}=\prod_{\mathfrak{p} \in F}\left(1-\mu_{\mathfrak{f}^{n} \mathfrak{p}} \mu_{\mathfrak{f}^{n} \mathfrak{p}}^{*}\right) .
$$

Since $\psi$ and $\varphi_{\beta}$ agree on $C_{1}$ (Lemma 4.4.5) and $F$ is finite, we obtain

$$
\psi\left(V_{n} V_{n}^{*}\right)=\varphi_{\beta}\left(\prod_{\mathfrak{p} \in F}\left(1-\mu_{\mathfrak{f} n} \mathfrak{p} \mu_{\mathfrak{f} n}^{*} \mathfrak{p}\right)\right) \stackrel{n \rightarrow \infty}{\longrightarrow} 1 .
$$

Now let $x \in C_{\nu}$. We want to prove that $\psi(x)=0$. For any $n \geqslant 1$, let $P_{n}=$ $1-V_{n} V_{n}^{*}$. The Schwarz inequality gives

$$
\left|\psi\left(P_{n} x\right)\right|^{2} \leqslant \psi\left(P_{n}\right) \psi\left(x x^{*}\right) .
$$

By equation (58), we have $\psi\left(P_{n}\right) \stackrel{n \rightarrow \infty}{\longrightarrow} 0$, so equation (59) gives $\psi\left(P_{n} x\right) \stackrel{n \rightarrow \infty}{\longrightarrow} 0$, so

$$
\psi\left(V_{n} V_{n}^{*} x\right) \stackrel{n \rightarrow \infty}{\longrightarrow} \psi(x) .
$$


For any $n \geqslant 1$, as $x \in C_{v}$ and $V_{n}^{*} \in C_{v^{-1}}$, we have $V_{n}^{*} x \in C_{1}$. Hence, by assumption, we have

$$
\psi\left(V_{n} V_{n}^{*} x\right)=0 .
$$

Together with equation (60), this gives $\psi(x)=0$, which completes the proof of Lemma 4.4.7.

Thus in order to prove that $\psi=\varphi_{\beta}$ it is enough to prove the following lemma.

Lemma 4.4.8. Let $v \in \overline{\operatorname{Gal}(K / k)}$ with $v \neq 1$. Let $F$ be a non-empty finite set of finite places of $k$ such that $v$ is $F$-localized. For any $F$-localized partial isometry $V \in C(X) \cap C_{v}$, we have

$$
\psi(V x)=0 \text { for all } x \in C_{1} .
$$

Proof. This proof is directly inspired by the proof of Lemma 27 (b) of [3]. It will make use of Lemmas 4.4.9, 4.4.10, 4.4.11, 4.4.12, 4.4.13 and 4.4.14, and will only be completed on p. 202.

Let $V \in C(X)$ be an $F$-localized partial isometry such that $V \in C_{\nu}$.

Let $E=V^{*} V=V V^{*}$ (the algebra $C(X)$ is commutative). Note that $E$ is a projection and belongs to $C_{1}$. Let

$$
C_{1, E}=E C_{1} E=\left\{f \in C_{1} \mid f=f E=E f\right\}
$$

denote the reduced algebra. As $V$ is fixed by the flow $\left(\sigma_{t}\right)$ and $\psi$ and $\varphi_{\beta}$ are $\operatorname{KMS}_{\beta}$ states for the flow $\left(\sigma_{t}\right)$, we see that $V$ belongs to the centralizer of $\psi$ and of $\varphi_{\beta}$.

Let $\alpha$ denote the following automorphism of $C_{1, E}$ :

$$
\alpha(f)=V f V^{*} \text { for all } f \in C_{1, E} .
$$

Let $M$ be the weak closure of $C_{k, \infty}$ in the GNS representation of $\varphi_{\beta}$. Let us extend the state $\varphi_{\beta}$ to a normal state $\widetilde{\varphi}_{\beta}$ on $M$. Let $M_{1} \subset M$ denote the weak closure of $C_{1}$ in the GNS representation of $\varphi_{\beta}$.

Since $V$ belongs to the centralizer of $\varphi_{\beta}$ for all $f \in C_{1, E}$, we have $\varphi_{\beta}(\alpha(f))=$ $\varphi_{\beta}(f)$. Thus $\alpha$ preserves $\varphi_{\beta}$, so it extends to an automorphism of the reduced algebra $M_{1, E}$ preserving $\widetilde{\varphi}_{\beta}$.

Let $c \in \mathfrak{I}_{\mathcal{O}}$ be an $F$-localized ideal such that $v$ factors through $\operatorname{Gal}\left(K_{\mathfrak{c}} / k\right)$.

Lemma 4.4.9. Let $\mathfrak{p}$ be a finite place of $k$ with $\mathfrak{p} \notin F$. We have

$$
E \mu_{\mathfrak{p}} \in C_{1, E} \quad \text { and } \quad \alpha\left(E \mu_{\mathfrak{p}}\right)=v\left(\sigma_{\mathfrak{p}}\right) E \mu_{\mathfrak{p}} \quad \text { for all } \mathfrak{p} \notin F,
$$

where $\sigma_{\mathfrak{p}}=\left(\mathfrak{p}, K_{F} / k\right) \in \operatorname{Gal}\left(K_{F} / k\right)$ is the Artin automorphism of $K_{F}$ associated to $\mathrm{p}$. 
Proof. Let $\mathscr{H}_{F}$ denote the $*$-algebra generated by the $e(\phi, \lambda)$, for all $\phi \in H(\mathrm{sgn})$ and all $\lambda \in \phi[F]$. By Proposition 4.4.3, we know that $\mathscr{H}_{F}$ is norm-dense in $C\left(X_{F}\right)$. So let $\left(V_{n}\right)_{n \in \mathbb{N}}$ be a sequence of elements of $\mathscr{H}_{F}$ converging to $V$ in the norm topology. Obviously we have

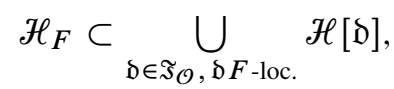

where $\mathfrak{D}$ runs over the $F$-localized elements of $\mathfrak{I}_{\mathcal{O}}$. Thus, for any $n \in \mathbb{N}$, there exists an $F$-localized $\mathfrak{\wp}_{n} \in \mathfrak{I}_{\mathcal{O}}$ such that $V_{n} \in \mathscr{H}\left[\mathfrak{D}_{n}\right]$. Since $\mathfrak{p} \notin F$ and $\mathfrak{D}_{n}$ is $F$-localized, we have $\mathfrak{p} \nmid \delta_{n}$, so Lemma 3.8.3 gives $V_{n} \mu_{\mathfrak{p}}=\mu_{\mathfrak{p}} \sigma_{\mathfrak{p}}\left(V_{n}\right)$. Now view $\sigma_{\mathfrak{p}}$ as an automorphism of the $\mathrm{C}^{*}$-algebra $C\left(X_{F}\right)$. In particular it is continuous. Hence we obtain

$$
V \mu_{\mathfrak{p}}=\mu_{\mathfrak{p}} \sigma_{\mathfrak{p}}(V)=v\left(\sigma_{\mathfrak{p}}\right) \mu_{\mathfrak{p}} V \quad \text { for all } \mathfrak{p} \notin F,
$$

and the result follows.

The ITPFI structure of $M_{1}$. For any $\mathfrak{p}$, recall that $\tau_{\mathfrak{p}}$ is the (Toeplitz) $\mathrm{C}^{*}$-algebra generated by $\mu_{\mathfrak{p}}$, and that $\varphi_{\beta, \mathfrak{p}}$ is the restriction of $\varphi_{\beta}$ to $\tau_{\mathfrak{p}}$. Let $\left(\varepsilon_{n}\right)_{n \geqslant 0}$ denote the standard orthonormal basis of $\ell^{2}(\mathbb{N})$. Let $\pi_{\beta, \mathfrak{p}}$ be the following representation of $\tau_{\mathfrak{p}}$ :

$$
\begin{aligned}
\pi_{\beta, \mathfrak{p}}: \tau_{\mathfrak{p}} & \rightarrow B\left(\ell^{2}(\mathbb{N}) \otimes \ell^{2}(\mathbb{N})\right), \\
\mu_{\mathfrak{p}} & \mapsto\left(\varepsilon_{n} \otimes \varepsilon_{m} \mapsto \varepsilon_{n+1} \otimes \varepsilon_{m}\right) .
\end{aligned}
$$

Let $\Omega_{\beta, p} \in \ell^{2}(\mathbb{N}) \otimes \ell^{2}(\mathbb{N})$ be the following vector:

$$
\Omega_{\beta, \mathfrak{p}}=\sqrt{1-\mathbf{N p}^{-\beta}} \sum_{n \geqslant 0} \mathbf{N p}^{-n \beta / 2} \varepsilon_{n} \otimes \varepsilon_{n} .
$$

It is easy to check that the pair $\left(\pi_{\beta, \mathfrak{p}}, \Omega_{\beta, \mathfrak{p}}\right)$ is the GNS representation of $\varphi_{\beta, \mathfrak{p}}$. Let $M_{1, \mathfrak{p}}$ denote the weak closure of $\tau_{\mathfrak{p}}$ in the representation $\pi_{\beta, \mathfrak{p}}$. One checks that

$$
M_{1, \mathfrak{p}}=B \ell^{2}(\mathbb{N}) \otimes \mathbb{C}
$$

In particular $M_{1, \mathfrak{p}}$ is a type $\mathrm{I}_{\infty}$ factor. Let $\tilde{\varphi}_{\beta, \mathfrak{p}}$ be the unique extension of $\varphi_{\beta, \mathfrak{p}}$ to a normal linear functional on $M_{1, \mathfrak{p}}$. Alternatively, $\tilde{\varphi}_{\beta, \mathfrak{p}}$ is the restriction of $\tilde{\varphi}_{\beta}$ to $M_{1, \mathfrak{p}}$. Note that the eigenvalue list of $\widetilde{\varphi}_{\beta, p}$ is the sequence

$$
\left(\left(1-\mathbf{N p}^{-\beta}\right) \mathbf{N p}^{-n \beta}\right)_{n \geqslant 0} .
$$

We have

$$
\left(M_{1}, \tilde{\varphi}_{\beta}\right)=\bigotimes_{\mathfrak{p}}\left(M_{1, \mathfrak{p}}, \tilde{\varphi}_{\beta, \mathfrak{p}}\right),
$$

where $\mathfrak{p}$ runs over the finite places of $k$. Recall from [33], III, Chapter XIV, Corollary 1.10 , that any ITPFI is a factor. In particular, $M_{1}$ is a factor. We shall check later (Lemma 4.5.1) that it is of type III $_{q-\beta}$. 
For any $\lambda \in \mathbb{C}$ with $|\lambda|=1$, let $\rho_{\mathfrak{p}, \lambda}$ denote the $*$-automorphism of $\tau_{\mathfrak{p}}$ such that $\rho_{\mathfrak{p}, \lambda}\left(\mu_{\mathfrak{p}}\right)=\lambda \mu_{\mathfrak{p}}$. As $\rho_{\mathfrak{p}, \lambda}$ preserves $\varphi_{\beta, \mathfrak{p}}$, it extends to an automorphism of $M_{1, \mathfrak{p}}$. Let $\theta_{F, v}$ be the following automorphism of $M_{1}$ :

$$
\theta_{F, v}=\left(\otimes_{\mathfrak{p} \in F} \operatorname{id}_{M_{1, \mathfrak{p}}}\right) \otimes\left(\otimes_{\mathfrak{p} \notin F} \rho_{\mathfrak{p}, v\left(\sigma_{\mathfrak{p}}\right)}\right) .
$$

Lemma 4.4.10. $\theta_{F, v}$ is an outer automorphism of $M_{1}$.

Proof. Suppose that $\theta_{F, v}$ is inner. Lemma 1.3.8 (b) from Connes [7] states that there exists a sequence $\left(u_{\mathfrak{p}}\right)$ where, for any finite place $\mathfrak{p}$ of $k, u_{\mathfrak{p}}$ is an unitary of $M_{1, \mathfrak{p}}$ with

$$
\theta_{F, v}(x)=u_{\mathfrak{p}} x u_{\mathfrak{p}}^{*} \quad \text { for all } x \in M_{1, \mathfrak{p}}
$$

and such that

$$
\sum_{\mathfrak{p}}\left(1-\left|\varphi_{\beta, \mathfrak{p}}\left(u_{\mathfrak{p}}\right)\right|\right)<\infty .
$$

Since $M_{1, \mathfrak{p}}$ is a factor, equation (65) determines $u_{\mathfrak{p}}$ up to multiplication by a $z \in \mathbb{C}$ with $|z|=1$. By definition of $\theta_{F, v}$, when $\mathfrak{p} \in F$ one can take $u_{\mathfrak{p}}=1$. When $\mathfrak{p} \notin F$ one can take $u_{\mathfrak{p}} \in M_{1, \mathfrak{p}}=B \ell^{2}(\mathbb{N})$ to be the diagonal matrix with eigenvalue list $\left(v\left(\sigma_{\mathfrak{p}}^{n}\right)\right)_{n \in \mathbb{N}}$. Using the expression of the GNS representation of $\varphi_{\beta, \mathfrak{p}}$ that we saw above, we get

$$
\varphi_{\beta, \mathfrak{p}}\left(u_{\mathfrak{p}}\right)=\left(1-\mathbf{N p}^{-\beta}\right) \sum_{n \in \mathbb{N}} v\left(\sigma_{\mathfrak{p}}\right)^{n} \mathbf{N p}^{-n \beta}=\frac{1-\mathbf{N p}^{-\beta}}{1-v\left(\sigma_{\mathfrak{p}}\right) \mathbf{N p}^{-\beta}} .
$$

This is equal to 1 whenever $v\left(\sigma_{\mathfrak{p}}\right)=1$; so, letting

$$
Y=\left\{\mathfrak{p} \mid \mathfrak{p} \text { is a finite place of } k, \mathfrak{p} \notin F \text {, and } v\left(\sigma_{\mathfrak{p}}\right) \neq 1\right\},
$$

equation (66) gives

$$
\sum_{\mathfrak{p} \in Y}\left(1-\left|\frac{1-\mathbf{N p}^{-\beta}}{1-v\left(\sigma_{\mathfrak{p}}\right) \mathbf{N} \mathfrak{p}^{-\beta}}\right|\right)<\infty
$$

Recall that we let $c$ be an $F$-localized ideal such that $v$ factors through $\operatorname{Gal}\left(K_{\mathfrak{c}} / k\right)$. This is a finite group, so the range of $\nu$ is finite, so there exists a $\gamma$ with $0<\gamma<1$ such that for any $\sigma \in \operatorname{Gal}(K / k)$ with $\nu(\sigma) \neq 1$, we have $\operatorname{Re} v(\sigma) \leqslant \gamma$. Let $\mathfrak{p} \in Y$. We have $\left|1-v\left(\sigma_{\mathfrak{p}}\right) \mathbf{N p}^{-\beta}\right| \geqslant 1-\gamma \mathbf{N p}^{-\beta}$. Thus we find

$$
1-\left|\frac{1-\mathbf{N} \mathfrak{p}^{-\beta}}{1-v\left(\sigma_{\mathfrak{p}}\right) \mathbf{N} \mathfrak{p}^{-\beta}}\right| \geqslant 1-\frac{1-\mathbf{N} \mathfrak{p}^{-\beta}}{1-\gamma \mathbf{N} \mathfrak{p}^{-\beta}}=\frac{(1-\gamma) \mathbf{N} \mathfrak{p}^{-\beta}}{1-\gamma \mathbf{N} \mathfrak{p}^{-\beta}} \geqslant(1-\gamma) \mathbf{N p}^{-\beta} .
$$

Since $1-\gamma>0$, together with equation (67) this gives

$$
\sum_{\mathfrak{p} \in Y} \mathbf{N p}^{-\beta}<\infty
$$


Since $\beta \leqslant 1$, this implies that for all $s \geqslant 1$ we have

$$
\sum_{\mathfrak{p} \in Y} \mathbf{N p}^{-s} \leqslant \sum_{\mathfrak{p} \in Y} \mathbf{N p}^{-1}<\infty .
$$

The Čebotarev density theorem (Theorem 1.1.4) states that for any $\sigma \in \operatorname{Gal}\left(K_{\mathrm{c}} / k\right)$ the following set $P_{\sigma}$ of places of $k$,

$$
P_{\sigma}=\left\{\mathfrak{p} \mid \mathfrak{p} \text { does not ramify in } K_{\mathfrak{c}} \text { and } \sigma_{\mathfrak{p}}=\sigma\right\},
$$

has a positive Dirichlet density

$$
d\left(P_{\sigma}\right)>0 .
$$

Up to a finite set of places of $k$, we have

$$
Y=\bigcup_{v(\sigma) \neq 1} P_{\sigma}
$$

where $\sigma$ runs over $\operatorname{Gal}\left(K_{\mathfrak{c}} / k\right)$. Hence, as $v \neq 1$, we have $d(Y)>0$, so

$$
\lim _{s \rightarrow 1_{+}} \sum_{\mathfrak{p} \in Y} \mathbf{N p}^{-s}=\infty,
$$

contradicting equation (69).

Define two subfactors $M_{1}^{F \pm}$ of $M_{1}$,

$$
\begin{aligned}
& M_{1}^{F+}=\bigotimes_{\mathfrak{p} \in F} M_{1, \mathfrak{p}}, \\
& M_{1}^{F-}=\bigotimes_{\mathfrak{p} \notin F}\left(M_{1, \mathfrak{p}}, \tilde{\varphi}_{\beta, \mathfrak{p}}\right),
\end{aligned}
$$

where $p$ runs over the finite places of $k$. We thus have

$$
M_{1}=M_{1}^{F+} \otimes M_{1}^{F-} .
$$

As the projection $E$ is $F$-localized, we have $E \in M_{1}^{F+}$, so letting

$$
N=\left(M_{1}^{F+}\right)_{E}
$$

and using [33], I, Chapter IV, Proposition 1.9, we get

$$
M_{1, E}=N \otimes M_{1}^{F-} .
$$

Lemma 4.4.11. $\alpha$ is an outer automorphism of $M_{1, E}$. 
Proof. Suppose that $\alpha$ is an inner automorphism of $M_{1, E}$. Let $\tau=\alpha^{-1} \circ \theta_{F, v} \in$ Aut $\left(M_{1, E}\right)$. By construction, $\tau$ induces the identity on $\mathbb{C} E \otimes M_{1}^{F-}$. As $N \otimes \mathbb{C}$ is of type $\mathrm{I}_{\infty}$, the restriction of $\tau$ to $N \otimes \mathbb{C}$ is inner. By equation (70), we get that $\tau$ is an inner automorphism of $M_{1, E}$. Hence, $\theta_{F, v}$ restricts to an inner automorphism of $M_{1, E}$. As $M_{1}$ is a factor, using Lemma 1.5.2 of [7], we deduce that $\theta_{F, v}$ is an inner automorphism of $M_{1}$, contradicting Lemma 4.4.10.

As we already noted, $V$ belongs to the centralizer of $\psi$. Define a linear functional $L$ on $C_{1, E}$ by

$$
L(x)=\psi(V x)=\psi(x V) \text { for all } x \in C_{1, E} .
$$

We want to prove by contradiction that $L$ is zero. Thus suppose that $L$ is nonzero.

The Schwarz inequality gives

$$
|L(x)|^{2} \leqslant \psi(E) \psi\left(x^{*} x\right) \quad \text { for all } x \in C_{1, E} .
$$

By Lemma 4.4.5, the states $\psi$ and $\varphi_{\beta}$ agree on $C_{1, E}$, so $\psi\left(x^{*} x\right)=\varphi_{\beta}\left(x^{*} x\right)$. Thus $L$ extends to a normal linear functional on $M_{1, E}$, which we still denote $L$.

Since $\varphi_{\beta}$ is $\mathrm{KMS}_{\beta}$ on $C_{k, \infty}$, by [4], II, Corollary 5.3.4 there exists a unique extension of $\left(\sigma_{t}\right)$ to an ultraweakly continuous flow $\left(\widetilde{\sigma}_{t}\right)$ on $M$ for which $\tilde{\varphi}_{\beta}$ is $\mathrm{KMS}_{\beta}$.

Lemma 4.4.12. The linear functional $L$ satisfies the $\alpha$-twisted $K M S_{\beta}$ condition for the flow $\left(\widetilde{\sigma}_{t}\right)$ on $M_{1, E}$. In other words, for any $x, y \in M_{1, E}$, there exists a bounded continuous function $F_{x, y}$ on the strip $0 \leqslant \operatorname{Im} z \leqslant \beta$, holomorphic on the interior of the strip, such that for any $t \in \mathbb{R}$ we have

$$
F_{x, y}(t)=L\left(x \sigma_{t}(y)\right) \text { and } F_{x, y}(t+i \beta)=L\left(\sigma_{t}(y) \alpha(x)\right) .
$$

Proof. In the case when $x, y \in C_{1, E}$, this can be easily checked by applying the $\mathrm{KMS}_{\beta}$ condition for $\psi$ to the pair $(V x, y)$. As both $\left(\widetilde{\sigma}_{t}\right)$ and $L$ are ultraweakly continuous, the result follows.

Lemma 4.4.13. There exists a nonzero $\widetilde{\sigma}_{t}$-invariant $w \in M_{1, E}$ such that

$$
L(x)=\tilde{\varphi}_{\beta}(w x) \text { for all } x \in M_{1, E} .
$$

Proof. Let $L=|L| u$ be the polar decomposition of $L$ (see [33], I, Chapter III, Theorem 4.2). In particular, $u \in M_{1, E}$ is a partial isometry, $|L|$ is a positive normal linear functional on $M_{1, E}$, and

$$
L(x)=|L|(u x) \quad \text { for all } x \in M_{1, E} .
$$

We want to apply Connes' Radon-Nikodým theorem to $|L|$ and $\widetilde{\varphi}_{\beta}$, seen as finite normal faithful weights on $M_{1, E}$. 
Since $L$ is $\widetilde{\sigma}_{t}$-invariant, by uniqueness of the polar decomposition, $u$ and $|L|$ are $\widetilde{\sigma}_{t^{-}}$invariant. As $\widetilde{\varphi}_{\beta}$ is $\mathrm{KMS}_{\beta}$ for the flow $\left(\widetilde{\sigma}_{t}\right)$, we deduce that $|L|$ is $\sigma_{t}^{\widetilde{\varphi}_{\beta}}$-invariant, where $\left(\sigma_{t}^{\widetilde{\varphi}_{\beta}}\right)$ is the modular automorphism group associated to the finite faithful normal weight $\tilde{\varphi}_{\beta}$ on $M_{1, E}$. Connes' Radon-Nikodým theorem ([7], Lemme 1.2.3 (b)) then states that there exists a positive $\widetilde{\sigma}_{t}$-invariant $h \in M_{1, E}$ such that

$$
|L|(x)=\tilde{\varphi}_{\beta}(h x) \text { for all } x \in M_{1, E} .
$$

Letting $w=h u$, we get

$$
L(x)=\tilde{\varphi}_{\beta}(w x) \text { for all } x \in M_{1, E},
$$

and $w$ is nonzero by our assumption that $L$ is nonzero. It is $\widetilde{\sigma}_{t}$-invariant because both $u$ and $h$ are.

Lemma 4.4.14. Let $w$ be given by Lemma 4.4.13. Then

$$
\alpha(x) w=w x \text { for all } x \in M_{1, E} .
$$

Proof. Let $x, y \in M_{1, E}$. Let $F_{x, y}^{L}$ be the function given by Lemma 4.4.12 such that for any $t \in \mathbb{R}$ we have

$$
F_{x, y}^{L}(t)=L\left(x \widetilde{\sigma}_{t}(y)\right) \quad \text { and } \quad F_{x, y}^{L}(t+i \beta)=L\left(\widetilde{\sigma}_{t}(y) \alpha(x)\right) .
$$

By definition of $w$, we get

$$
F_{x, y}^{L}(t)=\widetilde{\varphi}_{\beta}\left(w x \widetilde{\sigma}_{t}(y)\right) \quad \text { and } \quad F_{x, y}^{L}(t+i \beta)=\widetilde{\varphi}_{\beta}\left(\widetilde{\sigma}_{t}(w y) \alpha(x)\right) .
$$

Now let $F_{\alpha(x), w y}^{\widetilde{\varphi}_{\beta}}$ be the function given by the $\mathrm{KMS}_{\beta}$ property of $\widetilde{\varphi}_{\beta}$ applied to the pair $(\alpha(x), w y)$ so that

$$
F_{\alpha(x), w y}^{\widetilde{\varphi}_{\beta}}(t)=\widetilde{\varphi}_{\beta}\left(\alpha(x) \widetilde{\sigma}_{t}(w y)\right) \quad \text { and } \quad F_{\alpha(x), w y}^{\widetilde{\varphi}_{\beta}}(t+i \beta)=\widetilde{\varphi}_{\beta}\left(\widetilde{\sigma}_{t}(w y) \alpha(x)\right) .
$$

Let $G=F_{\alpha(x), w y}^{\widetilde{\varphi}_{\beta}}-F_{x, y}^{L}$. Note that $G$ vanishes on $\mathbb{R}+i \beta$. Therefore, one can extend $G$ to a holomorphic function on the broader strip $0<\operatorname{Im} z<2 \beta$ by letting

$$
G(z)=\overline{G(\bar{z}+2 i \beta)} \text { for all } z \in \mathbb{C} \text { with } \beta<\operatorname{Im} z<2 \beta .
$$

As $G$ vanishes on $\mathbb{R}+i \beta$ and is holomorphic on an open set containing $\mathbb{R}+i \beta$, it vanishes everywhere, so $F_{\alpha(x), w y}^{\widetilde{\varphi}_{\beta}}=F_{x, y}^{L}$. In particular, evaluating that at 0 , we get

$$
\tilde{\varphi}_{\beta}(w x y)=\tilde{\varphi}_{\beta}(\alpha(x) w y) .
$$

Since this holds for all $y \in M_{1, E}$ and the state $\widetilde{\varphi}_{\beta}$ is faithful on $M_{1, E}$, we get

$$
w x=\alpha(x) w .
$$


We already know by Lemma 4.4.11 that $\alpha$ is outer. Thus, Proposition 4.1.16 of Sunder [32] shows that

$$
\left\{y \in M_{1, E} \mid \alpha(x) y=y x \text { for all } x \in M_{1, E}\right\}=\{0\} .
$$

Together with Lemma 4.4.14 this shows that $w=0$. But $w$ is nonzero by construction (cf. Lemma 4.4.13), so we get a contradiction. Thus our assumption that $L$ is nonzero was false. Thus $L$ is zero, so

$$
\psi(V x)=0 \quad \text { for all } x \in C_{1, E} .
$$

Now let $x \in C_{1}$. We have $E x E \in C_{1, E}$, so it follows that $\psi(V E x E)=0$. As $E=V^{*} V=V V^{*}$ is a projection and belongs to the centralizer of $\psi$, we get $\psi(V E x E)=\psi(E V E x)=\psi(V x)$, so $\psi(V x)=0$, which proves Lemma 4.4.8.

From this we can deduce the main result of this subsection. Recall that we have assumed $0<\beta \leqslant 1$.

Theorem 4.4.15. The $C^{*}$-system $\left(C_{k, \infty}, \sigma_{t}\right)$ has exactly one $K M S_{\beta}$ state, $\varphi_{\beta}$.

Proof. This follows from Lemmas 4.4.6, 4.4.7 and 4.4.8.

Corollary 4.4.16. The state $\varphi_{\beta}$ of $C_{k, \infty}$ is a factor state, i.e., the von Neumann algebra $M$ is a factor.

Proof. This follows from Theorem 4.4.15 and [4], II, Theorem 5.3.30 (3).

4.5. The type $\mathrm{III}_{q^{-\beta}}$ of the $\mathrm{KMS}_{\beta}$ state at high temperature $1 / \beta \geqslant 1$. Let us go on with the notations of the preceding subsection. In particular, we assume that $\beta \leqslant 1$. The goal of this subsection is to prove (Theorem 4.5.8) that the state $\varphi_{\beta}$ on $C_{k, \infty}$ is of type III $_{q-\beta}$. In other words, we want to show that the factor $M$ is of type $\mathrm{III}_{q^{-\beta}}$. Before doing that we show that the subfactor $M_{1}$ is of type $\mathrm{III}_{q^{-\beta}}$.

Recall from equation (64) that $M_{1}$ is the following infinite tensor product, where $p$ runs over the finite places of $k$ :

$$
\left(M_{1}, \tilde{\varphi}_{\beta}\right)=\bigotimes_{\mathfrak{p}}\left(M_{1, \mathfrak{p}}, \tilde{\varphi}_{\beta, \mathfrak{p}}\right)
$$

Here each of the $M_{1, \mathfrak{p}}$ is a type $\mathrm{I}_{\infty}$ factor, so the usual methods (cf. Araki and Woods [1], [8]) allowing to compute asymptotic ratio sets cannot be applied directly to $M_{1}$. Instead we first find an integer $\tau \in \mathbb{N}$ and, for each $\mathfrak{p}$, a projection $e_{\mathfrak{p}} \in M_{1, \mathfrak{p}}$ such that the reduced factor $M_{1, \mathfrak{p}, e_{\mathfrak{p}}}$ is of type $\mathrm{I}_{\tau}$ and the infinite tensor product $e=\otimes_{\mathfrak{p}} e_{\mathfrak{p}}$ is a nonzero projection in $M_{1}$. 
Let $\tau \in \mathbb{N}$ be such that $\tau>1 / \beta$. For any finite place $\mathfrak{p}$ of $k$, let $e_{\mathfrak{p}}=1-\mu_{\mathfrak{p}}^{\tau} \mu_{\mathfrak{p}}^{* \tau} \in$ $M_{1, \mathfrak{p}}$. Recall from equation (63) that $M_{1, \mathfrak{p}}$ is naturally identified with $B \ell^{2}(\mathbb{N})$. Under this identification, the projection $e_{\mathfrak{p}}$ is the diagonal matrix whose $\tau$ first diagonal entries are 1 and whose other entries are 0 . Thus, the reduced subfactor $M_{1, \mathfrak{p}, e_{\mathfrak{p}}}$ is of type $\mathrm{I}_{\tau}$. Note that

$$
\tilde{\varphi}_{\beta, \mathfrak{p}}\left(e_{\mathfrak{p}}\right)=1-\mathbf{N p}^{-\tau \beta} .
$$

Any decreasing sequence of projections in a von Neumann algebra converges weakly to a projection, so we can define a projection $e \in M_{1}$ by

$$
e=\prod_{\mathfrak{p}} e_{\mathfrak{p}}=\bigotimes_{\mathfrak{p}} e_{\mathfrak{p}}
$$

By definition of $\tau$ we have $\tau \beta>1$, so

$$
\tilde{\varphi}_{\beta}(e)=\prod_{\mathfrak{p}} \tilde{\varphi}_{\beta, \mathfrak{p}}\left(e_{\mathfrak{p}}\right)=\prod_{\mathfrak{p}}\left(1-\mathbf{N p}^{-\tau \beta}\right)=\frac{1}{\zeta_{k, \infty}(\tau \beta)} \neq 0 .
$$

In particular, $e \neq 0$. Let us define a state $\widetilde{\varphi}_{\beta, e}$ on $M_{1, e}$ by

$$
\tilde{\varphi}_{\beta, e}(x)=\zeta_{k, \infty}(\tau \beta) \tilde{\varphi}_{\beta}(x)=\frac{\tilde{\varphi}_{\beta}(x)}{\tilde{\varphi}_{\beta}(e)} \quad \text { for all } x \in M_{1, e} .
$$

For any $\mathfrak{p}$, let us define a state $\widetilde{\varphi}_{\beta, \mathfrak{p}, e_{\mathfrak{p}}}$ on $M_{1, \mathfrak{p}, e_{\mathfrak{p}}}$ by

$$
\tilde{\varphi}_{\beta, \mathfrak{p}, e_{\mathfrak{p}}}(x)=\left(1-\mathbf{N} \mathfrak{p}^{-\tau \beta}\right)^{-1} \widetilde{\varphi}_{\beta, \mathfrak{p}}(x)=\frac{\widetilde{\varphi}_{\beta, \mathfrak{p}}(x)}{\widetilde{\varphi}_{\beta, \mathfrak{p}}\left(e_{\mathfrak{p}}\right)} \quad \text { for all } x \in M_{1, \mathfrak{p}, e_{\mathfrak{p}}} .
$$

Coming back to the definition of the infinite tensor product ([33], III, Chapter XIV, §1) and using the expression (64) of $M_{1}$ as the infinite tensor product of the $\left(M_{1, \mathfrak{p}}, \tilde{\varphi}_{\beta, \mathfrak{p}}\right)$, one can check that

$$
\left(M_{1, e}, \tilde{\varphi}_{\beta, e}\right)=\bigotimes_{\mathfrak{p}}\left(M_{1, \mathfrak{p}, e_{\mathfrak{p}}}, \widetilde{\varphi}_{\beta, \mathfrak{p}, e_{\mathfrak{p}}}\right) .
$$

Let $\left(\sigma_{t}^{\widetilde{\varphi}_{\beta}}\right)$ denote the modular flow of $\widetilde{\varphi}_{\beta}$. Since $\widetilde{\varphi}_{\beta}$ is $\mathrm{KMS}_{\beta}$ for the flow $\left(\widetilde{\sigma}_{t}\right)$, we have

$$
\sigma_{t}^{\tilde{\varphi}_{\beta}}=\tilde{\sigma}_{\beta t} \quad \text { for all } t \in \mathbb{R} .
$$

Lemma 4.5.1. The factor $M_{1}$ is of type $\mathrm{III}_{q^{-\beta}}$.

Proof. Let us first prove that $q^{-\beta}$ belongs to the asymptotic ratio set $r_{\infty}\left(M_{1, e}\right)$. We want to apply the criterion given on p. 465 of [8] to the ITPFI in equation (73). The eigenvalue list of $\tilde{\varphi}_{\beta, \mathfrak{p}, e_{\mathfrak{p}}}$ is $\left(\lambda_{\mathfrak{p}, a}\right)_{a=0, \ldots, \tau-1}$, where

$$
\lambda_{\mathfrak{p}, a}=\frac{\left(1-\mathbf{N} \mathfrak{p}^{-\beta}\right) \mathbf{N} \mathfrak{p}^{-a \beta}}{1-\mathbf{N p}^{-\tau \beta}} .
$$


Let $r$ be such that $0<r<1$. For any $n \in \mathbb{N}$, let

$$
r(n)=\left\lfloor r q^{n} / n\right\rfloor .
$$

By equation (1), there exists an $n_{0} \geqslant 1$ such that for any $n \geqslant n_{0}$, there exist (at least) $r(n)$ distinct finite places

$$
\mathfrak{p}_{n}^{1}, \ldots, \mathfrak{p}_{n}^{r(n)}
$$

of $k$ such that

$$
\mathbf{N} \mathfrak{p}_{n}^{i}=q^{n} \quad \text { for all } i \in\{1, \ldots, r(n)\} .
$$

For any $n \geqslant n_{0}$, let $I_{n}$ be the following set of places of $k$ :

$$
I_{n}=\left\{\mathfrak{p}_{2 n}^{1}, \ldots, \mathfrak{p}_{2 n}^{r(2 n)}, \mathfrak{p}_{2 n+1}^{1}, \ldots, \mathfrak{p}_{2 n+1}^{r(2 n)}\right\} .
$$

Let $X\left(I_{n}\right)=\{0, \ldots, \tau-1\}^{I_{n}}$ be the set of all maps from $I_{n}$ to $\{0, \ldots, \tau-1\}$. Define a measure $\lambda$ on $X\left(I_{n}\right)$ by

$$
\lambda(\{f\})=\prod_{i=1}^{r(2 n)} \lambda_{\mathfrak{p}_{2 n}^{i}, f\left(\mathfrak{p}_{2 n}^{i}\right)} \lambda_{\mathfrak{p}_{2 n+1}^{i}, f\left(\mathfrak{p}_{2 n+1}^{i}\right)} .
$$

For any $i \in\{1, \ldots, r(2 n)\}$, define elements $k_{n}^{1, i}$ and $k_{n}^{2, i}$ of $X\left(I_{n}\right)$ by

$$
k_{n}^{1, i}(\mathfrak{p})=1_{\mathfrak{p}=\mathfrak{p}_{2 n}^{i}} \quad \text { and } \quad k_{n}^{2, i}(\mathfrak{p})=1_{\mathfrak{p}=\mathfrak{p}_{2 n+1}^{i}} \quad \text { for all } \mathfrak{p} \in I_{n} .
$$

Let $K_{n}^{1}=\left\{k_{n}^{1,1}, \ldots, k_{n}^{1, r(2 n)}\right\}$ and let $K_{n}^{2}=\left\{k_{n}^{2,1}, \ldots, k_{n}^{2, r(2 n)}\right\}$. For any $i \in\{1, \ldots, r(2 n)\}$, we have $\lambda\left(\left\{k_{n}^{1, i}\right\}\right)=\lambda\left(\left\{k_{n}^{1,1}\right\}\right)$, so

$$
\begin{aligned}
\lambda\left(K_{n}^{1}\right) & =r(2 n) \lambda\left(\left\{k_{n}^{1,1}\right\}\right) \\
& =r \cdot\left(\frac{1-q^{-2 n \beta}}{1-q^{-2 n \tau \beta}}\right)^{\left\lfloor r q^{2 n} /(2 n)\right\rfloor}\left(\frac{1-q^{-(2 n+1) \beta}}{1-q^{-(2 n+1) \tau \beta}}\right)^{\left\lfloor r q^{2 n /(2 n)\rfloor}\right.} \frac{\left(q^{1-\beta}\right)^{2 n}}{(2 n)} .
\end{aligned}
$$

Since $\beta \leqslant 1$, one checks easily that

$$
\sum_{n \geqslant n_{0}} \lambda\left(K_{n}^{1}\right)=\infty
$$

Let $\phi_{n}: K_{n}^{1} \rightarrow K_{n}^{2}$ be the bijection defined by $\phi_{n}\left(k_{n}^{1, i}\right)=k_{n}^{2, i}$. For any $i \in$ $\{1, \ldots, 2 n\}$, we have

$$
\frac{\lambda\left(\left\{\phi_{n}\left(k_{n}^{1, i}\right)\right\}\right)}{\lambda\left(\left\{k_{n}^{1, i}\right\}\right)}=\frac{\lambda_{\mathfrak{p}_{2 n}^{i}, 0} \lambda_{p_{2 n+1}^{i}, 1}}{\lambda_{\mathfrak{p}_{2 n}^{i}, 1} \lambda_{\mathfrak{p}_{2 n+1}^{i}, 0}}=\frac{q^{-(2 n+1) \beta}}{q^{-2 n \beta}}=q^{-\beta} .
$$


Together with equation (75), this allows to apply the criterion given on p. 465 of [8], and we get

$$
q^{-\beta} \in r_{\infty}\left(M_{1, e}\right)
$$

Hence, by [7], Théorème 3.6.1, we have $q^{-\beta} \in S\left(M_{1, e}\right)$. Hence, by [7], Corollaire 3.2.8 (b), we have

$$
q^{-\beta} \in S\left(M_{1}\right)
$$

In particular, this shows that $S\left(M_{1}\right) \neq\{0,1\}$, so, by [7], Théorème 3.4.1, one gets that $S\left(M_{1}\right) \cap \mathbb{R}_{+}^{*}$ is the orthogonal of $T\left(M_{1}\right)$ for the duality $(s, t) \mapsto s^{i t}$. By construction, $\widetilde{\sigma}_{2 \pi / \log q}=1$, so equation (74) gives

$$
\sigma_{2 \pi /(\beta \log q)}^{\widetilde{\varphi}_{\beta}}=1 .
$$

Thus

$$
T\left(M_{1}\right) \supset \frac{2 \pi}{\beta \log q} \mathbb{Z} .
$$

Hence, by orthogonality, we get

$$
S\left(M_{1}\right) \cap \mathbb{R}_{+}^{*} \subset q^{\beta \mathbb{Z}} .
$$

Since we already know that $q^{-\beta} \in S\left(M_{1}\right)$, we obtain

$$
S\left(M_{1}\right) \cap \mathbb{R}_{+}^{*}=q^{\beta \mathbb{Z}} .
$$

Thus $M_{1}$ is of type $\mathrm{III}_{q^{-\beta}}$.

We only use Lemma 4.5 .1 for the proof the following corollary. Let $M_{1, \widetilde{\varphi}_{\beta}}$ denote the centralizer of $\widetilde{\varphi}_{\beta}$ in $M_{1}$.

Corollary 4.5.2. The centralizer $M_{1, \tilde{\varphi}_{\beta}}$ is a factor, of type $\mathrm{II}_{1}$.

Proof. Lemma 4.5.1 and equation (76) allow to apply [7], Théorème 4.2.6, and we obtain that $M_{1, \widetilde{\varphi}_{\beta}}$ is a factor. Note that $\widetilde{\varphi}_{\beta}$ is a finite faithful normal trace on $M_{1, \widetilde{\varphi}_{\beta}}$. Hence, the type of $M_{1, \widetilde{\varphi}_{\beta}}$ can only be either $\mathrm{II}_{1}$ or $\mathrm{I}_{n}$ with $n \in \mathbb{N}^{*}$. Let $\mathfrak{p}$ be a finite place of $k$. For any $n \geqslant 1$, set $x_{n}=\mu_{\mathfrak{p}}^{n} \mu_{\mathfrak{p}}^{* n}$. Note that the $x_{n}$ are fixed by the flow $\left(\sigma_{t}\right)$, hence by equation (74) they belong to $M_{1, \widetilde{\varphi}_{\beta}}$. Equation (33) shows that the $x_{n}$ are linearly independent over $\mathbb{C}$. Thus, $M_{1, \tilde{\varphi}_{\beta}}$ is infinite-dimensional over $\mathbb{C}$, so its type cannot be $\mathrm{I}_{n}$ with $n \in \mathbb{N}^{*}$. Hence, it must be $\mathrm{II}_{1}$.

Our next goal is to prove (Lemma 4.5.6) that the centralizer $M_{\tilde{\varphi}_{\beta}}$ of $\tilde{\varphi}_{\beta}$ in $M$ is also a factor.

Definition 4.5.3. For any $\delta \in \mathfrak{I}_{\mathcal{O}}$, let $M[\delta]$ denote the weak closure of $\mathscr{H}[\delta]$ in $M$. 
Lemma 4.5.4. Let $\mathfrak{D} \in \mathfrak{I}_{\mathcal{O}}$. Let $\mathfrak{p}$ be a maximal ideal of $\mathfrak{I}_{\mathcal{O}}$ not dividing $\mathfrak{D}$. Let $\sigma_{\mathfrak{p}}=\left(\mathfrak{p}, K_{\mathfrak{D}} / k\right) \in \operatorname{Gal}\left(K_{\mathfrak{D}} / k\right)$ be the Artin automorphism of $K_{\mathfrak{D}}$ associated to $\mathfrak{p}$. Then:

(1) The automorphism $\sigma_{\mathfrak{p}}$ of $\mathscr{H}[\S]$ extends uniquely to an ultraweakly continuous automorphism of $M[\delta]$.

(2) For all $x \in M[\delta]$, we have

$$
x \mu_{\mathfrak{p}}=\mu_{\mathfrak{p}} \sigma_{\mathfrak{p}}(x) .
$$

Proof. Let us first prove (1). Uniqueness is clear because, by the von Neumann density theorem, $\mathscr{H}[§]$ is ultraweakly dense in $M[\S]$. Let $\sigma \in \mathrm{Gal}(K / k)$ be such that $\left.\sigma\right|_{K_{\mathfrak{D}}}=\sigma_{\mathfrak{p}}$. As $\varphi_{\beta} \circ \sigma=\varphi_{\beta}$ on $C_{k, \infty}$, we know that $\sigma$ extends to an ultraweakly continuous automorphism of $M$, which we still note $\sigma$. The required extension of $\sigma_{\mathfrak{p}}$ is then obtained by taking the restriction of $\sigma$ to $M[\delta]$.

Let us now check (2). By density, it is enough to check equation (77) when $x \in \mathcal{H}[\delta]$. It then follows from Lemma 3.8.3.

Lemma 4.5.5. Let $\downarrow \in \mathfrak{I}_{\mathcal{O}}$. Let $M[\S]_{\tilde{\varphi}_{\beta}}$ denote the centralizer of $\widetilde{\varphi}_{\beta}$ in $M[\delta]$. Let $Z\left(M[\S]_{\tilde{\varphi}_{\beta}}\right)$ denote the center of $M[\S]_{\tilde{\varphi}_{\beta}}$. Then:

$$
Z\left(M[\delta]_{\tilde{\varphi}_{\beta}}\right) \subset M_{1} .
$$

Proof. Let $x$ belong to $Z\left(M[§]_{\tilde{\varphi}_{\beta}}\right)$. As $x$ belongs to $M[\S]$, it is fixed by $\operatorname{Gal}\left(K / K_{\triangleright}\right)$. Let $\sigma \in \operatorname{Gal}\left(K_{\mathfrak{D}} / k\right)=\operatorname{Gal}(K / k) / \operatorname{Gal}\left(K / K_{\mathfrak{D}}\right)$. By Corollary 1.1.5, there exist finite places $\mathfrak{p}, \mathfrak{q}$ of $k$ not dividing $\mathfrak{D}$ such that $\mathbf{N} \mathfrak{p}=\mathbf{N}_{\mathfrak{q}}, \sigma_{\mathfrak{p}}=\sigma$ and $\sigma_{\mathfrak{q}}=1$. Since $\mathbf{N} \mathfrak{p}=\mathbf{N} \mathfrak{q}$, we have

$$
\sigma_{t}\left(\mu_{\mathfrak{p}} \mu_{\mathfrak{q}}^{*}\right)=\mathbf{N} \mathfrak{p}^{i t} \mathbf{N q}_{\mathfrak{q}}^{-i t} \mu_{\mathfrak{p}} \mu_{\mathfrak{q}}^{*}=\mu_{\mathfrak{p}} \mu_{\mathfrak{q}}^{*} \quad \text { for all } t \in \mathbb{R} .
$$

Hence, by equation (74), $\mu_{\mathfrak{p}} \mu_{\mathfrak{q}}^{*} \in M[\S]_{\tilde{\varphi}_{\beta}}$. Thus, as $x$ belongs to the center of $M[\delta]_{\tilde{\varphi}_{\beta}}$, we have

$$
x \mu_{\mathfrak{p}} \mu_{\mathfrak{q}}^{*}=\mu_{\mathfrak{p}} \mu_{\mathfrak{q}}^{*} x .
$$

On the other hand, by Lemma 4.5.4 (2), we have

$$
x \mu_{\mathfrak{p}} \mu_{\mathfrak{q}}^{*}=\mu_{\mathfrak{p}} \sigma_{\mathfrak{p}}(x) \mu_{\mathfrak{q}}^{*},
$$

and we also compute

$$
\begin{aligned}
\mu_{\mathfrak{p}} \mu_{\mathfrak{q}}^{*} x & =\mu_{\mathfrak{p}}\left(x^{*} \mu_{\mathfrak{q}}\right)^{*} \\
& =\mu_{\mathfrak{p}}\left(\mu_{\mathfrak{q}} \sigma_{\mathfrak{q}}\left(x^{*}\right)\right)^{*} \quad \text { by Lemma 4.5.4 (2) } \\
& =\mu_{\mathfrak{p}} \sigma_{\mathfrak{q}}(x) \mu_{\mathfrak{q}}^{*} .
\end{aligned}
$$


Combining equations (78), (79), and (80), we get

$$
\mu_{\mathfrak{p}} \sigma_{\mathfrak{p}}(x) \mu_{\mathfrak{q}}^{*}=\mu_{\mathfrak{p}} \sigma_{\mathfrak{q}}(x) \mu_{\mathfrak{q}}^{*} .
$$

Multiplying both sides of equation (81) by $\mu_{\mathfrak{p}}^{*}$ on the left and by $\mu_{\mathfrak{q}}$ on the right, and applying relation $\left(a_{1}\right)$ of Proposition 3.1.2, we get

$$
\sigma_{\mathfrak{p}}(x)=\sigma_{\mathfrak{q}}(x) .
$$

Since $\sigma_{\mathfrak{p}}=\sigma$ and $\sigma_{\mathfrak{q}}=1$, we get

$$
\sigma(x)=x .
$$

Thus, $x \in M_{1}$.

Let $M_{\tilde{\varphi}_{\beta}}$ denote the centralizer of $\tilde{\varphi}_{\beta}$ in $M$.

Lemma 4.5.6. The centralizer $M_{\widetilde{\varphi}_{\beta}}$ is a factor of type $\mathrm{II}_{1}$.

Proof. Note that $\widetilde{\varphi}_{\beta}$ is a finite, faithful, normal, positive, normalized trace on $M_{\widetilde{\varphi}_{\beta}}$. Let $\operatorname{tr}$ be another such trace on $M_{\tilde{\varphi}_{\beta}}$. Let us prove that $\operatorname{tr}=\tilde{\varphi}_{\beta}$. Let $\delta \in \mathfrak{\Im}_{\mathcal{O}}$. By Connes' Radon-Nikodým theorem, [7], Lemme 1.2.3 (b), there exists a positive element $h$ of $M[\delta]_{\tilde{\varphi}_{\beta}}$ such that

$$
\operatorname{tr}(x)=\tilde{\varphi}_{\beta}(h x) \quad \text { for all } x \in M[\S]_{\tilde{\varphi}_{\beta}} .
$$

Since $\tilde{\varphi}_{\beta}$ and tr are faithful traces, one easily checks that $h$ belongs to the center $Z\left(M[\delta]_{\tilde{\varphi}_{\beta}}\right)$. Thus, by Lemma 4.5.5, $h \in M_{1}$. Hence, the restriction of tr to $M[\delta]_{\tilde{\varphi}_{\beta}}$ is $\operatorname{Gal}(K / k)$-invariant, so

$$
\operatorname{tr}(x)=\operatorname{tr}(\mathbf{E}(x)) \text { for all } x \in M[\delta]_{\tilde{\varphi}_{\beta}} .
$$

As $\left(\sigma_{t}^{\tilde{\varphi}_{\beta}}\right)$ is $(2 \pi / \log q)$-periodic, we have a normal conditional expectation

$$
\begin{aligned}
E_{\widetilde{\varphi}_{\beta}}: M & \rightarrow M_{\widetilde{\varphi}_{\beta}}, \\
x & \mapsto \frac{\log q}{2 \pi} \int_{0}^{2 \pi / \log q} \sigma_{t}^{\widetilde{\varphi}_{\beta}}(x) d t .
\end{aligned}
$$

Since $\mathscr{H}$ is norm-dense in $C_{k, \infty}$ (see Proposition 3.3.5), it is ultraweakly dense in $M$, and it follows that $E_{\widetilde{\varphi}_{\beta}}(\mathscr{H})$ is ultraweakly dense in $E_{\widetilde{\varphi}_{\beta}}(M)$. We have

$$
\begin{aligned}
E_{\widetilde{\varphi}_{\beta}}(\mathcal{H}) & =E_{\widetilde{\varphi}_{\beta}}\left(\bigcup_{\delta \in \Im_{\mathcal{O}}} \mathcal{H}[\delta]\right) \subset E_{\widetilde{\varphi}_{\beta}}\left(\bigcup_{\delta \in \Im_{\mathcal{O}}} M[\delta]\right) \\
& \subset \bigcup_{\delta \in \mathfrak{I}_{\mathcal{O}}} E_{\widetilde{\varphi}_{\beta}}(M[\delta]) \subset \bigcup_{\delta \in \mathfrak{I}_{\mathcal{O}}} M[\delta]_{\widetilde{\varphi}_{\beta}} .
\end{aligned}
$$


Thus, $\bigcup_{\mathfrak{D} \in \mathfrak{I}_{\mathcal{O}}} M[\S]_{\widetilde{\varphi}_{\beta}}$ is ultraweakly dense in $E_{\widetilde{\varphi}_{\beta}}(M)=M_{\widetilde{\varphi}_{\beta}}$. Thus equation (82) gives

$$
\operatorname{tr}(x)=\operatorname{tr}(\mathbf{E}(x)) \text { for all } x \in M_{\tilde{\varphi}_{\beta}} .
$$

We know by Corollary 4.5.2 that $M_{1, \widetilde{\varphi}_{\beta}}$ is a type $\mathrm{II}_{1}$ factor. Hence, by Jones [22], Corollary 7.1.19, we know that $\operatorname{tr}$ and $\widetilde{\varphi}_{\beta}$ agree on $M_{1, \widetilde{\varphi}_{\beta}}$. Thus, by equation (83), we deduce that $\operatorname{tr}$ and $\widetilde{\varphi}_{\beta}$ agree on $M_{\widetilde{\varphi}_{\beta}}$. Hence, by [22], Corollary 7.1.20, we deduce that $M_{\widetilde{\varphi}_{\beta}}$ is a factor, and the same argument that we made for $M_{1, \widetilde{\varphi}_{\beta}}$ shows that $M_{\widetilde{\varphi}_{\beta}}$ is also of type $\mathrm{II}_{1}$.

Corollary 4.5.7. We have $S(M) \neq\{0,1\}$. In other words, the factor $M$ is not of type $\mathrm{III}_{0}$.

Proof. Suppose that $S(M)=\{0,1\}$. Then, by [7], Corollaire 3.2.7 (b), the center of $M_{\tilde{\varphi}_{\beta}}$ has no minimal nonzero projection. Hence, by Lemma 4.5.6, one deduces that $\mathbb{C}$ has no minimal nonzero projection, which is absurd.

Finally we can prove the main result of this subsection. Recall that we have assumed $0<\beta \leqslant 1$.

Theorem 4.5.8. The state $\varphi_{\beta}$ on $C_{k, \infty}$ is of type $\mathrm{III}_{q^{-\beta}}$. In other words, the factor $M$ is of type $\mathrm{III}_{q^{-\beta}}$.

Proof. By Corollary 4.5.7 and [7], Théorème 3.4.1, the set $S(M) \cap \mathbb{R}_{+}^{*}$ is the orthogonal of $T(M)$ for the duality $(s, t) \mapsto s^{i t}$. Hence, it is enough to prove that

$$
T(M)=\frac{2 \pi}{\beta \log q} \cdot \mathbb{Z} .
$$

Since $\widetilde{\sigma}_{2 \pi / \log q}=1$, equation (74) gives

$$
\frac{2 \pi}{\beta \log q} \in T(M)
$$

which proves one inclusion. Let us prove the other one. Let $t_{0} \in \mathbb{R}$ be such that $t_{0} / \beta \in T(M)$. Thus, by equation (74), $\widetilde{\sigma}_{t_{0}}$ is an inner automorphism of $M$. Let $u$ be an unitary of $M$ such that

$$
\widetilde{\sigma}_{t_{0}}(x)=u x u^{*} \text { for all } x \in M .
$$

For any $t \in \mathbb{R}$ and $x \in M$, we have

$$
\widetilde{\sigma}_{t}(u) \widetilde{\sigma}_{t}(x) \widetilde{\sigma}_{t}(u)^{*}=\widetilde{\sigma}_{t_{0}+t}\left(u x u^{*}\right)=u \widetilde{\sigma}_{t}(x) u^{*},
$$


so the unitaries $u$ and $\sigma_{t}(u)$ implement the same inner automorphism of the factor $M$, so there exists some $z_{t} \in \mathbb{C}$ with $\left|z_{t}\right|=1$ and $\sigma_{t}(u)=z_{t} u$. The map $t \mapsto z_{t}$ is a character of $\mathbb{R}$, so there exists $\theta \in \mathbb{R}$ such that

$$
z_{t}=e^{i \theta t} \quad \text { for all } t \in \mathbb{R} .
$$

The $\mathrm{KMS}_{\beta}$ property of the state $\tilde{\varphi}_{\beta}$ for the flow $\left(\widetilde{\sigma}_{t}\right)$ applied to the pair $\left(u^{*}, u\right)$ gives a bounded continuous function $F$ on the strip $0 \leqslant \operatorname{Im} z \leqslant \beta$, holomorphic on the interior of the strip, such that

$$
F(t)=\tilde{\varphi}_{\beta}\left(u^{*} \sigma_{t}(u)\right) \quad \text { and } \quad F(t+i \beta)=\tilde{\varphi}_{\beta}\left(\sigma_{t}(u) u^{*}\right) \quad \text { for all } t \in \mathbb{R} \text {. }
$$

Thus,

$$
F(t)=e^{i \theta t}=F(t+i \beta) \text { for all } t \in \mathbb{R} .
$$

Hence $F$ is the holomorphic function $z \mapsto e^{i \theta z}$ and, evaluating equation (84) at $t=0$, one gets

$$
e^{-\theta \beta}=1
$$

Thus $\theta=0$, so $u$ is fixed by the flow $\left(\widetilde{\sigma}_{t}\right)$. Hence, by equation (74), the unitary $u$ belongs to the centralizer $M_{\widetilde{\varphi}_{\beta}}$ of $\tilde{\varphi}_{\beta}$. Moreover, by equation (74), any element of $M_{\widetilde{\varphi}_{\beta}}$ is fixed by the flow $\left(\widetilde{\sigma}_{t}\right)$ and so commutes with $u$, by definition of $u$. Hence $u$ belongs to the center of $M_{\tilde{\varphi}_{\beta}}$. Thus, by Lemma 4.5.6, one deduces that $u \in \mathbb{C}$, so, as an automorphism of $M$,

$$
\tilde{\sigma}_{t_{0}}=1 \text {. }
$$

By equation (1), for any sufficiently large $n$, there exist finite places $\mathfrak{p}$ and $\mathfrak{q}$ of $k$ such that $\mathbf{N} \mathfrak{p}=q^{n}$ and $\mathbf{N q}=q^{n+1}$. We then have $\sigma_{t_{0}}\left(\mu_{\mathfrak{q}} \mu_{\mathfrak{p}}^{*}\right)=q^{(n+1) i t_{0}-n i t_{0}} \mu_{\mathfrak{q}} \mu_{\mathfrak{p}}^{*}=$ $q^{i t_{0}} \mu_{\mathfrak{q}} \mu_{\mathfrak{p}}^{*}$. On the other hand, equation (85) gives $\sigma_{t_{0}}\left(\mu_{\mathfrak{q}} \mu_{\mathfrak{p}}^{*}\right)=\mu_{\mathfrak{q}} \mu_{\mathfrak{p}}^{*}$. Thus, we get $1=q^{i t_{0}}$, so $t_{0} \in 2 \pi /(\log q) \mathbb{Z}$, which completes the proof.

\section{References}

[1] H. Araki and E. J. Woods, A classification of factors. Publ. Res. Inst. Math. Sci. 4 (1968/1969), 51-130. Zbl 0206.12901 MR 0244773

[2] J. Arledge, M. Laca, and I. Raeburn, Semigroup crossed products and Hecke algebras arising from number fields. Doc. Math. 2 (1997), 115-138. Zbl 0940.47062 MR 1451963

[3] J.-B. Bost and A. Connes, Hecke algebras, type III factors and phase transitions with spontaneous symmetry breaking in number theory. Selecta Math. (N.S.) 1 (1995), 411-457. Zbl 0842.46040 MR 1366621

[4] O. Bratteli and D. W. Robinson, Operator algebras and quantum-statistical mechanics. I, II. 2nd ed., Springer-Verlag, New York 1987, Berlin 1997. Zbl 0905.46046 Zbl 0903.46066; MR 0887100 MR 1441540 
[5] J. W. S. Cassels and A. Fröhlich (eds.), Algebraic number theory. Academic Press, London 1967. Zbl 0153.07403 MR 0215665

[6] P. B. Cohen, A $C^{*}$-dynamical system with Dedekind zeta partition function and spontaneous symmetry breaking. J. Théor. Nombres Bordeaux 11 (1999), 15-30.Zbl 0962.11031 MR 1730430

[7] A. Connes, Une classification des facteurs de type III. Ann. Sci. École Norm. Sup. (4) 6 (1973), 133-252. Zbl 0274.46050 MR 0341115

[8] A. Connes, Noncommutative geometry. Academic Press, San Diego, CA, 1994. Zbl 0818.46076 MR 1303779

[9] A. Connes, Trace formula in noncommutative geometry and the zeros of the Riemann zeta function. Selecta Math. (N.S.) 5 (1999), 29-106. Zbl 0945.11015 MR 1694895

[10] A. Connes, C. Consani, and M. Marcolli, Noncommutative geometry and motives: the thermodynamics of endomotives. Adv. in Math., to appear; arXiv:math.QA/0512138

[11] A. Connes and M. Marcolli, Quantum statistical mechanics of $\mathbb{Q}$-lattices (from physics to number theory via noncommutative geometry, part I). In Frontiers in Number Theory, Physics, and Geometry I (P. Cartier, B. Julia, P. Moussa, P. Vanhove, eds.), second printing, Springer-Verlag, Berlin 2006, 269-349. MR 2261099

[12] A. Connes, M. Marcolli, and N. Ramachandran, KMS states and complex multiplication. Selecta Math. (N.S.) 11 (2005), 325-347. Zbl 05081957 MR 2215258

[13] A. Connes, M. Marcolli, and N. Ramachandran, KMS states and complex multiplication. Part II. In Operator Algebras, Abel Symposia 1, Springer-Verlag, Berlin 2006, 15-59.

[14] K. R. Davidson, $C^{*}$-algebras by example. Fields Inst. Monogr. 6, Amer. Math. Soc., Providence, RI, 1996. Zbl 0958.46029 MR 1402012

[15] D. Goss, Basic structures of function field arithmetic. Ergeb. Math. Grenzgeb. (3) 35, Springer-Verlag, Berlin 1996. Zbl 0892.11021 MR 1423131

[16] E. Ha and F. Paugam, Bost-Connes-Marcolli systems for Shimura varieties. Part I. Definitions and formal analytic properties. Internat. Math. Res. Papers 2005 (2005), 237-286. Zbl 05034362 MR 2199962

[17] D. Harari and E. Leichtnam, Extension du phénomène de brisure spontanée de symétrie de Bost-Connes au cas des corps globaux quelconques. Selecta Math. (N.S.) 3 (1997), 205-243. Zbl 0924.46051 MR 1466166

[18] D. R. Hayes, Explicit class field theory for rational function fields. Trans. Amer. Math. Soc. 189 (1974), 77-91. Zbl 0292.12018 MR 0330106

[19] D. R. Hayes, Explicit class field theory in global function fields. In Studies in algebra and number theory, Adv. Math., Suppl. Stud. 6, Academic Press, New York 1979, 173-217. Zbl 0476.12010 MR 0535766

[20] D. R. Hayes, A brief introduction to Drinfel'd modules, In The arithmetic of function fields, Ohio State Univ. Math. Res. Inst. Publ. 2, de Gruyter, Berlin 1992, 1-32. Zbl 0793.11015 MR 1196509

[21] M. Jarden, The Čebotarev density theorem for function fields: an elementary approach. Math. Ann. 261 (1982), 467-475. Zbl 0501.12018 MR 682659 
[22] V. F. R. Jones, Von Neumann algebras. Course at Berkeley University, 2003.

[23] A. L. Kholodenko, Statistical mechanics of $2+1$ gravity from Riemann zeta function and Alexander polynomial: exact results. J. Geom. Phys. 38 (2001), 81-139. Zbl 0990.83004 MR 1823664

[24] M. Khoshkam and G. Skandalis, Regular representation of groupoid $C^{*}$-algebras and applications to inverse semigroups. J. Reine Angew. Math. 546 (2002), 47-72. Zbl 1029.46082 MR 1900993

[25] M. Laca, Semigroups of *-endomorphisms, Dirichlet series, and phase transitions. $J$. Funct. Anal. 152 (1998), 330-378. Zbl 0957.46039 MR 1608003

[26] M. Laca and I. Raeburn, The ideal structure of the Hecke $C^{*}$-algebra of Bost and Connes. Math. Ann. 318 (2000), 433-451. Zbl 1032.46536 MR 1800765

[27] M. Laca and M. van Frankenhuijsen, Phase transitions on Hecke $C^{*}$-algebras and classfield theory over $\mathbb{Q}$.J. Reine Angew. Math. 595 (2006), 25-53.Zbl 05039453 MR 2244797

[28] N. S. Larsen and I. Raeburn, Representations of Hecke algebras and dilations of semigroup crossed products. J. London Math. Soc. (2) 66 (2002), 198-212. Zbl 1023.46074 MR 1911869

[29] S. Neshveyev, Ergodicity of the action of the positive rationals on the group of finite adeles and the Bost-Connes phase transition theorem. Proc. Amer. Math. Soc. 130 (2002), 2999-3003. Zbl 1031.46077 MR 1908923

[30] G. K. Pedersen, $C^{*}$-algebras and their automorphism groups. London Math Soc. Monogr. 14, Academic Press, London 1979. Zbl 0416.46043 MR 0548006

[31] J. Renault, A groupoid approach to $C^{*}$-algebras. Lecture Notes in Math. 793, SpringerVerlag, Berlin 1980. Zbl 0433.46049 MR 0584266

[32] V. S. Sunder, An invitation to von Neumann algebras. Universitext, Springer-Verlag, New York 1987. Zbl 0616.46052 MR 0866671

[33] M. Takesaki, Theory of operator algebras. I-III. Springer-Verlag, New York, Berlin 1979, 2003. Zbl 0990.46034, Zbl 1059.46031 Zbl 1059.46032; MR 0548728 MR 1943006 MR 1943007

[34] A. Weil, Sur les courbes algébriques et les variétés qui s'en déduisent. Hermann, Paris 1948. Zbl 0036.16001 MR 0027151

Received April 21, 2006

Projet Algèbres d'Opérateurs, Institut de Mathématiques de Jussieu, 175 rue du Chevaleret, 75013 Paris, France

E-mail: jacob@math.jussieu.fr 